\title{
Measurement of the properties of a Higgs boson in the four-lepton final state
}

\author{
S. Chatrchyan et al. \\ (CMS Collaboration) \\ (Received 18 December 2013; published 14 May 2014)
}

\begin{abstract}
The properties of a Higgs boson candidate are measured in the $H \rightarrow Z Z \rightarrow 4 \ell$ decay channel, with $\ell=e, \mu$, using data from $p p$ collisions corresponding to an integrated luminosity of $5.1 \mathrm{fb}^{-1}$ at the centerof-mass energy of $\sqrt{s}=7 \mathrm{TeV}$ and $19.7 \mathrm{fb}^{-1}$ at $\sqrt{s}=8 \mathrm{TeV}$, recorded with the CMS detector at the LHC. The new boson is observed as a narrow resonance with a local significance of 6.8 standard deviations, a measured mass of $125.6 \pm 0.4$ (stat) \pm 0.2 (syst) $\mathrm{GeV}$, and a total width $\leq 3.4 \mathrm{GeV}$ at the $95 \%$ confidence level. The production cross section of the new boson times its branching fraction to four leptons is measured to be $0.93_{-0.23}^{+0.26}$ (stat $)_{-0.09}^{+0.13}$ (syst) times that predicted by the standard model. Its spin-parity properties are found to be consistent with the expectations for the standard-model Higgs boson. The hypotheses of a pseudoscalar and all tested spin-1 boson hypotheses are excluded at the $99 \%$ confidence level or higher. All tested spin-2 boson hypotheses are excluded at the $95 \%$ confidence level or higher.
\end{abstract}

DOI: 10.1103/PhysRevD.89.092007

PACS numbers: $14.80 . \mathrm{Bn}, 12.15 . \mathrm{Ji}, 13.85 . \mathrm{Rm}$

\section{INTRODUCTION}

The standard model (SM) of particle physics [1-5] describes very successfully the electroweak and strong interactions of elementary particles over a wide range of energies. In the SM, the massive mediators of the electroweak force, the $W$ and $Z$ bosons, acquire mass through the mechanism of spontaneous symmetry breaking [6-11]. This mechanism introduces a complex scalar field with four degrees of freedom, three of which lead to the $W$ and $Z$ bosons acquiring mass, while the fourth gives rise to a physical particle, the scalar Higgs boson $H$. The masses of the fermions arise through Yukawa interactions between the fermions and the scalar field $[12,13]$. The mass of the Higgs boson $m_{H}$ is a free parameter of the model and has to be determined experimentally. General theoretical considerations on the unitarity of the SM [14-17] suggest that $m_{H}$ should be smaller than $\approx 1 \mathrm{TeV}$, while precision electroweak measurements imply that $m_{H}<152 \mathrm{GeV}$ at the $95 \%$ confidence level (C.L.) [18]. Using about $5 \mathrm{fb}^{-1}$ of data collected at $\sqrt{s}=7 \mathrm{TeV}$ in 2011 and about $5 \mathrm{fb}^{-1}$ of additional data collected in the first half of 2012 at $\sqrt{s}=8 \mathrm{TeV}$, the ATLAS and CMS experiments have reported the discovery of a new boson at a mass around $125 \mathrm{GeV}$, with properties compatible with those of the SM Higgs boson [19-21]. Previously, direct searches for the Higgs boson have been carried out at the LEP collider, leading to a lower bound of $m_{H}>114.4 \mathrm{GeV}$ at the 95\% C.L. [22], and at the Tevatron proton-antiproton collider, excluding the mass ranges $90-109 \mathrm{GeV}$ and

$\bar{*}$ Full author list given at the end of the article.

Published by the American Physical Society under the terms of the Creative Commons Attribution 3.0 License. Further distribution of this work must maintain attribution to the author(s) and the published articles title, journal citation, and DOI.
$149-182 \mathrm{GeV}$ at the $95 \%$ C.L. and indicating a broad excess of events in the range $120-135 \mathrm{GeV}[23,24]$.

Searches for the SM Higgs boson in the $H \rightarrow Z Z \rightarrow 4 \ell$ $(\ell=e, \mu)$ channel at the Large Hadron Collider (LHC) have been previously performed using a sample corresponding to an integrated luminosity of about $5 \mathrm{fb}^{-1}$ of 2011 data by the ATLAS [25-27] and Compact Muon Solenoid (CMS) [28-30] collaborations. After the new boson discovery, the spin-parity properties have been further studied by both experiments, using more data. The pseudoscalar hypothesis is excluded by CMS [31] and ATLAS experiments [32,33] at the 95\% C.L. or higher. ATLAS has also excluded at the $99 \%$ C.L. the hypotheses of vector, pseudovector, and graviton-like spin2 bosons, under certain assumptions on their production mechanisms [33].

In this paper, the analysis of the $H \rightarrow Z Z \rightarrow 4 \ell$ channel is presented using the entire data set collected by the CMS experiment during the 2011-2012 LHC running period. The data correspond to an integrated luminosity of $5.1 \mathrm{fb}^{-1}$ of $p p$ collisions at a center-of-mass energy of $\sqrt{s}=7 \mathrm{TeV}$, and $19.7 \mathrm{fb}^{-1}$ at $\sqrt{s}=8 \mathrm{TeV}$. The search looks for a signal consisting of two pairs of same-flavor, opposite-charge, well-identified and isolated leptons, $e^{+} e^{-}$, $\mu^{+} \mu^{-}$, compatible with a $Z Z$ system, where one or both of the $Z$ bosons can be off shell, appearing as a narrow resonance on top of a smooth background in the four-lepton invariant mass distribution. Improved calibrations and alignment constants with respect to those used in Refs. [20,21,31], based on the full data set, are used in the reconstruction of the events considered for this paper. The statistical significance of the observation of the new boson in the four-lepton decay mode is reported, together with measurements of the boson's mass and its cross section times its branching fraction with respect to the SM prediction, an upper limit on the boson's width, and the 
compatibility of the boson with nine alternative spin-parity hypotheses. The compatibility of the data with a mixed scalar/pseudoscalar state is also assessed. A search is also conducted for additional resonances compatible with the SM Higgs boson in the $H \rightarrow Z Z \rightarrow 4 \ell$ channel in the mass range $110-1000 \mathrm{GeV}$.

The paper is organized as follows: The apparatus, the data samples, and the online selection are described in Secs. II through IV. Sections V through VII describe the reconstruction and identification algorithms used in this analysis for leptons, photons, and jets. The event selection and categorization are discussed in Sec. VIII. The background estimation is described in Sec. IX. Kinematic discriminants used to further improve the separation between signal and background and to test the spin and parity of the new boson are presented in Sec. X. The event yields, kinematic distributions, and measured properties are discussed in Secs. XI through XIII.

\section{THE CMS DETECTOR}

The central feature of the CMS apparatus is a superconducting solenoid of $6 \mathrm{~m}$ internal diameter, providing a $3.8 \mathrm{~T}$ field. Within the superconducting solenoid volume are a silicon pixel and strip tracker, a lead tungstate crystal electromagnetic calorimeter (ECAL), and a brass/scintillator hadron calorimeter (HCAL). Muons are detected in gas-ionization detectors embedded in the iron flux return placed outside the solenoid. Extensive forward calorimetry complements the coverage provided by the barrel and end-cap detectors. The CMS detector is described in detail in Ref. [34].

The CMS experiment uses a coordinate system with the origin at the nominal interaction point, the $x$ axis pointing to the center of the LHC ring, the $y$ axis pointing up (perpendicular to the LHC ring), and the resulting $z$ axis along the beam direction using a right-handed convention. The polar angle $\theta$ is measured from the positive $z$ axis, and the azimuthal angle $\phi$ is measured in the $x-y$ plane in radians. The pseudorapidity is defined as $\eta=-\ln [\tan (\theta / 2)]$.

The inner tracker measures charged particle trajectories within the range $|\eta|<2.5$. It consists of 1440 silicon pixel and 15148 silicon strip detector modules and is immersed in the magnetic field. It provides an impact parameter resolution of $\approx 15 \mu \mathrm{m}$ and a transverse momentum $\left(p_{\mathrm{T}}\right)$ resolution of about $1.5 \%$ for $100 \mathrm{GeV}$ particles [35,36].

The ECAL consists of 75848 lead tungstate crystals and provides coverage of $|\eta|<1.479$ in the barrel region (EB), and $1.479<|\eta|<3.0$ in the two end-cap regions (EE). The EB uses $23 \mathrm{~cm}$ long crystals with front-face cross sections of around $2.2 \mathrm{~cm} \times 2.2 \mathrm{~cm}$, while the EE comprises $22 \mathrm{~cm}$ long crystals with front-face cross sections of $2.86 \mathrm{~cm} \times 2.86 \mathrm{~cm}$. A preshower detector consisting of two planes of silicon sensors interleaved with a total of 3 radiation lengths of lead is located in front of the EE. The
ECAL energy resolution for electrons with transverse energy $E_{\mathrm{T}} \approx 45 \mathrm{GeV}$ from the $Z \rightarrow e^{+} e^{-}$decays is better than $2 \%$ in the central region of the $\mathrm{EB}(|\eta|<0.8)$, and is between $2 \%$ and $5 \%$ elsewhere. For low-bremsstrahlung electrons that have $94 \%$ or more of their energy contained within a $3 \times 3$ array of crystals, the energy resolution improves to $1.5 \%$ for $|\eta|<0.8$ [37]. The Gaussian resolution of the dielectron mass distribution for a $Z$-boson sample, when both electrons belong to this class, is $0.97 \pm 0.01 \mathrm{GeV}$ in $\sqrt{s}=7 \mathrm{TeV}$ data.

The HCAL is a sampling calorimeter with brass as the passive material and plastic scintillator tiles serving as active material, providing coverage of $|\eta|<2.9$. The calorimeter cells are grouped in projective towers of granularity $\Delta \eta \times \Delta \phi=0.087 \times 0.087$ in the HB (covering $|\eta|<1.3$ ) and $\Delta \eta \times \Delta \phi \approx 0.17 \times 0.17$ in the HE (covering $1.3<|\eta|<2.9$ ), the exact granularity depending on $|\eta|$. A hadron forward calorimeter extends the coverage up to $|\eta|<5.2$.

Muons are detected in the pseudorapidity range $|\eta|<2.4$, with detection planes made using three technologies: drift tubes, cathode-strip chambers, and resistiveplate chambers. The global fit of the muon tracks matched to the tracks reconstructed in the silicon tracker results in a transverse momentum resolution, averaged over $\phi^{\mu}$ and $\eta^{\mu}$, from $1.8 \%$ at $p_{\mathrm{T}}^{\mu}=30 \mathrm{GeV}$ to $2.3 \%$ at $p_{\mathrm{T}}^{\mu}=50 \mathrm{GeV}$ [36].

\section{SIMULATED DATA SAMPLES}

The Monte Carlo (MC) simulated samples, generated with programs based on state-of-the-art theoretical calculations for both the SM Higgs boson signal and relevant background processes, are used to optimize the event selection and to evaluate the acceptance and systematic uncertainties. The samples of Higgs boson signal events produced in either gluon fusion $(g g \rightarrow H)$ or vector-boson fusion $(q q \rightarrow q q H)$ processes are generated with the POWHEG [38-40] generator at next-to-leading-order (NLO) QCD accuracy. The Higgs boson decay is modeled with JHUGEN 3.1.8 [41-43] and includes proper treatment of interference effects associated with permutations of identical leptons in the four-electron and four-muon final states. Alternative spin-parity states are also modeled with JHUGEN, where production of the spin- 0 states is modeled in gluon fusion with POWHEG at NLO QCD accuracy. It is also found that NLO QCD effects relevant for this analysis are approximated well with the combination of leadingorder (LO) QCD matrix elements and parton showering. Therefore, simulation of spin- 1 and spin- 2 resonances is performed in quark-antiquark and gluon fusion production at LO QCD accuracy, followed by parton showering generated with PYTHIA 6.4.24 [44].

For low-mass Higgs boson hypotheses $\left(m_{H}<400 \mathrm{GeV}\right)$, the Higgs boson line shape is described with a Breit-Wigner (BW) distribution. At high mass $\left(m_{H} \geq 400 \mathrm{GeV}\right)$, because of the very large Higgs boson width $\left(\Gamma_{H}>70 \mathrm{GeV}\right)$, the 
line shape is described using the complex pole scheme (CPS) [45-47]. The inclusive cross section for every $m_{H}$ is computed including corrections due to the CPS [48]. The interference between the Higgs boson signal produced by gluon fusion and the background from $g g \rightarrow Z Z$ is taken into account, as suggested in Ref. [49]. The theoretical uncertainty in the shape of the resonance due to missing NLO corrections in the interference between background and signal is considered, as well as the uncertainties due to electroweak corrections $[46,49,50]$. Samples of $W H$, $Z H$, and $t \bar{t} H$ events are generated with PYTHIA. Higgs boson signal events for all the production mechanisms are reweighted using the generator-level invariant mass, to include contributions from gluon fusion up to next-tonext-to-leading order (NNLO) and next-to-next-to-leading logarithm (NNLL) [51-63], and from the vector-boson fusion (VBF) contribution computed at NNLO in Refs. [55,64-68].

The dominant background to the Higgs signal in this channel is the $\mathrm{SM} Z Z$ or $Z \gamma^{*}$ production via $q \bar{q}$ annihilation and gluon fusion, which is referred to as $Z Z$ in what follows. Smaller contributions arise from $Z+$ jets and $t \bar{t}$ production where the final states contain two isolated leptons and two heavy-flavor jets producing secondary leptons. Additional backgrounds arise from $Z+$ jets, $Z \gamma+$ jets, $W W+$ jets, and $W Z+$ jets events, where misidentified leptons can arise from decays of heavy-flavor hadrons, in-flight decays of light mesons within jets, and, in the case of electrons, overlaps of $\pi^{0}$ decays with charged hadrons. The $Z Z$ production via $q \bar{q}$ is generated at NLO with POWHEG [69], while the $W W, W Z$ processes are generated with MADGRAPH [70] and normalized to cross sections computed at NLO. The $g g \rightarrow Z Z$ contribution is generated with GG2ZZ [71]. The $Z b \bar{b}, Z c \bar{c}, Z \gamma$, and $Z+$ light jets samples (referred to as $Z+$ jets in the following) are generated with MADGRAPH, comprising inclusive $Z$ production of up to four additional partons at the matrixelement level, which is normalized to the cross section computed at NNLO. The $t \bar{t}$ events are generated at NLO with POWHEG. The event generator takes into account the internal initial-state and final-state radiation effects which can lead to the presence of additional hard photons in an event. In the case of LO generators, the CTEQ6L [72] set of parton distribution functions (PDFs) is used, while the CT10 [73] set is used for the NLO and higher-order generators.

All generated samples are processed with PYTHIA for jet fragmentation and showering. For the underlying event, the PYTHIA 6.4.24 tunes Z2 and Z2*, which rely on $p_{\mathrm{T}}$-ordered showers, are used for 7 and $8 \mathrm{TeV}$ MC samples, respectively [74]. Events are processed through the detailed simulation of the CMS detector based on GEANT4 $[75,76]$ and are reconstructed with the same algorithms as used for data. The simulations include overlapping $p p$ interactions (pileup) matching the distribution of the number of interactions per LHC beam crossing observed in data. The average number of measured pileup interactions is approximatively 9 and 21 in the 7 and $8 \mathrm{TeV}$ data sets, respectively.

\section{ONLINE EVENT SELECTION}

The first level (L1) of the CMS trigger system, composed of custom hardware processors, uses information from the calorimeters and muon detectors to select the most interesting events in a time interval of less than $4 \mu \mathrm{s}$. The L1 trigger rate of $100 \mathrm{kHz}$ is further reduced by the high-level trigger (HLT) processor farm to around $300 \mathrm{~Hz}$ before data storage.

Collision events analyzed in this paper are selected by the trigger system, requiring the presence of two leptons: electrons or muons. The minimal transverse momenta of the leading and subleading leptons are 17 and $8 \mathrm{GeV}$, respectively, for both electrons and muons. The online selection includes double-electron, double-muon and mixed electron-muon triggers. In the case of the $4 e$ final state, a triple-electron trigger is added with thresholds of 15,8 , and $5 \mathrm{GeV}$ to increase the efficiency for low- $p_{\mathrm{T}}$ electrons. The trigger efficiency for events within the geometrical acceptance of this analysis is greater than $98 \%$ for a Higgs boson signal with $m_{H}>110 \mathrm{GeV}$. The same trigger paths are applied on the 7 and $8 \mathrm{TeV}$ data, whereas different identification criteria are applied on the HLT lepton candidates to account for the different LHC conditions.

In addition to the events selected to form the four-lepton sample, dedicated triggers are used for lepton calibration and efficiency measurements. In the case of dimuon events, the online trigger algorithms used to select the signal events are sufficiently loose that they can also be used to measure the selection efficiency with the $Z \rightarrow \mu^{+} \mu^{-}$events. In order to measure the selection efficiency of events with low- $p_{\mathrm{T}}$ leptons, low-mass resonances are used. Events corresponding to these low-mass resonances are collected in the dimuon case using dedicated triggers that require an opposite-sign muon pair, with dedicated kinematic conditions on the dimuon system. In the case of electrons, low-mass resonances are collected, with a smaller rate, with standard dielectron triggers. Two specialized triggers are introduced to maximize the number of $Z \rightarrow e^{+} e^{-}$events covering both high- and low- $p_{\mathrm{T}}$ ranges. The one having the most stringent (relaxed) identification and isolation requirement on one electron requires the presence of a cluster in the electromagnetic calorimeter with $p_{\mathrm{T}}>8(17) \mathrm{GeV}$, forming an invariant mass with the other electron exceeding $50 \mathrm{GeV}$.

\section{LEPTON RECONSTRUCTION AND SELECTION}

The analysis is performed by reconstructing a $Z Z$ system composed of two pairs of same-flavor and opposite-charge 
isolated leptons, $e^{+} e^{-}$or $\mu^{+} \mu^{-}$. The main background sources, described in Sec. III, are the SM ZZ production, with smaller contributions from other diboson $(W W, W Z)$ processes, single bosons with hadronic activity that can mimic lepton signatures, and top-quark-pair events. Given the very low branching fraction of the $H \rightarrow Z Z \rightarrow 4 \ell$ decay, of $\mathcal{O}\left(10^{-4}\right)\left[\mathcal{O}\left(10^{-3}\right)\right]$ for $m_{H}=125(200) \mathrm{GeV}$ [77], it is important to maintain a very high lepton selection efficiency in a wide range of momenta, to maximize the sensitivity for a Higgs boson within the mass range $110-1000 \mathrm{GeV}$.

The signal sensitivity also depends on the $4 \ell$ invariant mass resolution. The signal appears as a narrow resonance on top of a smooth background, and therefore it is important to achieve the best possible four-lepton mass resolution. To obtain a precise measurement of the mass of a resonance decaying into four leptons, it is crucial to calibrate the individual lepton momentum scale and resolution to a level such that the systematic uncertainty in the measured value of $m_{H}$ is substantially smaller than the statistical uncertainty in the current data set. This section describes the techniques used in the analysis to select electrons and muons in order to achieve the best momentum resolution, measure the momentum scale, resolution, and selection efficiency, and derive corrections based on dilepton resonances.

The CMS particle flow (PF) algorithm [78-81], which combines information from all subdetectors, is used to provide an event description in the form of reconstructed particle candidates. The PF candidates are then used to build higher-level objects, such as jets, missing transverse energy, and lepton isolation quantities.

\section{A. Electron reconstruction and identification}

Electron candidates are required to have a transverse momentum $p_{\mathrm{T}}^{e}>7 \mathrm{GeV}$ and be within the geometrical acceptance, defined by $\left|\eta^{e}\right|<2.5$. The electron reconstruction combines information from the ECAL and the tracker [82-85]. Electron candidates are formed from arrays of energy clusters in the ECAL (called superclusters) along the $\phi$ direction, which are matched to tracks in the silicon tracker. Superclusters, which recover the energy of the bremsstrahlung photons emitted in the tracker material and of some of the nearly collinear final-state radiation (FSR) from the electron, are also used to identify hits in the innermost tracker layers in order to initiate the reconstruction of electron tracks. This track seeding procedure is complemented by an approach based on tracker seeds which improves the reconstruction efficiency at low $p_{\mathrm{T}}^{e}$ and in the transition between the EB and EE regions. Trajectories, when initiated outside-in from the ECAL superclusters as well as inside-out from the measurements in the innermost tracker layers, are reconstructed using the Gaussian sum filter (GSF) algorithm [86], which accounts for the electron energy loss by bremsstrahlung. Additional requirements [37] are applied in order to reject electrons originating from photon conversions in the tracker material. Electron candidates are selected using loose criteria on track-supercluster matching observables that preserve the highest possible efficiency while removing part of the QCD background.

Electron identification relies on a multivariate discriminant that combines observables sensitive to the bremsstrahlung along the electron trajectory, and the geometrical and momentum-energy matching between the electron trajectory and the associated supercluster, as well as ECAL shower-shape observables. The multivariate discriminant is trained using a sample of $\approx 10^{7}$ simulated Drell-Yan events for the signal (true electrons) and a high-purity $W+1$ jet data sample for the background (misidentified electrons from jets). The expected performance is validated using jets misidentified as electrons in a $Z\left(\rightarrow \mu^{+} \mu^{-}\right)$and $Z\left(\rightarrow e^{+} e^{-}\right)$ data sample, with exactly one reconstructed electron not originated from the $Z$ boson decay. The sources of prompt electrons, such as dibosons or $t \bar{t}$ decays, are suppressed with appropriate selections on the number of extra leptons and the presence of small missing transverse energy in the event [85]. The selection of the $Z$ boson is the same as the one used in the analysis, so the $\eta^{e}$ and $p_{\mathrm{T}}^{e}$ spectrum is similar to the one for the electrons characterizing the reducible background in the analysis. The selection is optimized in six regions of the electron $p_{\mathrm{T}}^{e}$ and $\left|\eta^{e}\right|$ to maximize the expected sensitivity for a low-mass Higgs boson. These regions correspond to two $p_{\mathrm{T}}^{e}$ ranges, 7-10 GeV and $>10 \mathrm{GeV}$, and three pseudorapidity regions, corresponding to two regions in the EB with different material in front of the ECAL, the central barrel $\left(\left|\eta^{e}\right|<0.8\right)$ and the outer barrel $\left(0.800<\left|\eta^{e}\right|<1.479\right)$, in addition to the EE, $1.479<\left|\eta^{e}\right|<2.500$.

Several procedures are used to calibrate the energy response of individual crystals [37,87]. The energy of the ECAL superclusters is corrected for the imperfect containment of the clustering algorithm, the electron energy not deposited in the ECAL, and leakage arising from showers near gaps between crystals or between ECAL modules. This is done using a regression technique based on boosted decision trees (BDT) [88] trained on a simulated dielectron sample with the pileup conditions equivalent to the ones measured on data, covering a flat spectrum in $p_{\mathrm{T}}^{e}$ from 5 to $100 \mathrm{GeV}$. The variables include the electron supercluster raw energy, $\eta$ and $\phi$ coordinates, several shower-shape variables of the cluster with largest energy within the supercluster (the seed cluster), the ratio of the energy in the HCAL behind the seed cluster to the seed cluster energy, and the number of clusters in the electron supercluster. In addition, the distance of the seed crystal with respect to the gap between the ECAL modules, the $\eta$ and $\phi$ coordinates of the seed cluster, and the energies of the first three subleading clusters in the supercluster are used. A similar subset of variables is used depending on 
whether the electron is detected in the EB or EE. Using this multivariate technique, the effective width and Gaussian resolution of the reconstructed invariant mass are improved by $25 \%$ and $30 \%$, respectively, for simulated $H \rightarrow 4 e$ decays compared to those obtained with a more traditional approach based on ECAL-only energy measurements and corrections with a parameterized energy response obtained from simulation. The effective width, $\sigma_{\text {eff }}$, is defined as the half-width of the smallest interval that contains $68.3 \%$ of the distribution.

The precision of the electron momentum measurement is dominated by the ECAL at high energies, whereas for low- $p_{\mathrm{T}}$ electrons the precision is dominated by the tracker momentum determination. Moreover, for electrons near poorly instrumented regions, such as the crack between the EB and the EE, the intermodule cracks [89], or regions close to dead channels, the measurement accuracy and resolution can also be improved by combining the ECAL energy with the track momentum. To account for biases arising from bremsstrahlung losses in the tracker material, electron categories are defined based on the cluster multiplicity inside the supercluster as well as on the amount of bremsstrahlung as estimated from the GSF. The magnitude of the electron momentum is then determined by combining the two estimates with a multivariate regression function that takes as input the corrected ECAL energy from the supercluster regression, the track momentum estimate, their respective uncertainties, the ratio of the corrected ECAL energy over the track momentum as obtained from the track fit, the uncertainty in this ratio, and the electron category, based on the amount of bremsstrahlung. The direction is taken from the fitted track parameters at the point of closest approach to the nominal beam spot position. Figure 1 (top) shows the reconstructed invariant mass for $H \rightarrow 4 e$ decays, compared to the traditional approach for the electron energy estimation. The residual offset in the peak position $[<0.2 \%$, black histogram in Fig. 1 (top)] is irrelevant for the analysis, because the absolute electron momentum scale is calibrated using known resonances in data, as described in Sec. VD. Figure 1 (bottom) presents the expected effective resolution of the combined momentum measurement as a function of the electron momentum at the vertex. The expected effective momentum resolution for the ECAL-only and tracker-only estimates is also shown.

\section{B. Muon reconstruction and identification}

Muon candidates are required to have a transverse momentum $p_{\mathrm{T}}^{\mu}>5 \mathrm{GeV}$ and be within the geometrical acceptance, defined by $\left|\eta^{\mu}\right|<2.4$. The reconstruction combines information from both the silicon tracker and the muon system. The matching between track segments is done either outside-in, starting from a track in the muon system, or inside-out, starting from a track in the silicon tracker. Both these candidates are referred to as global
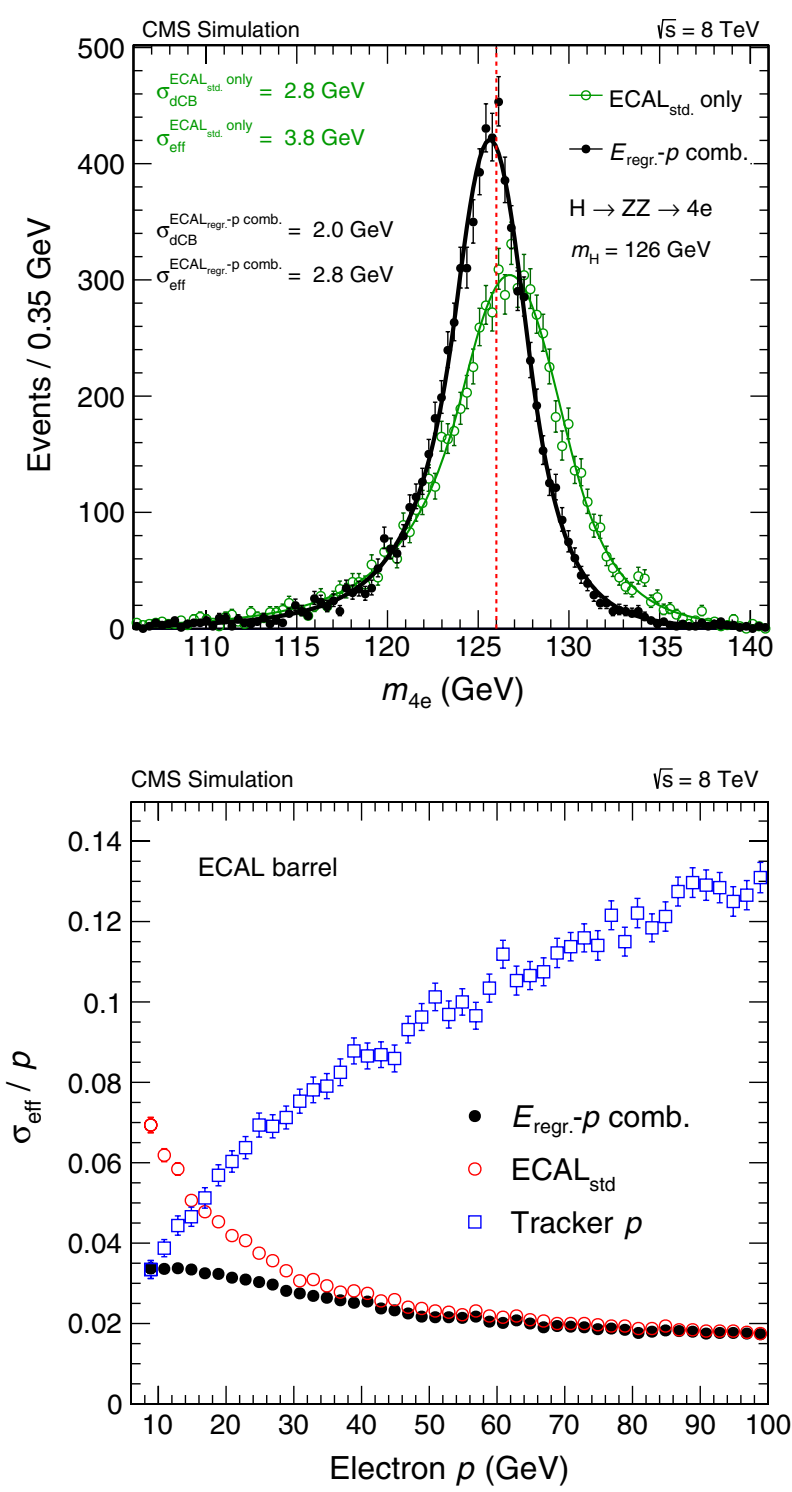

FIG. 1 (color online). (top) Expected four-lepton mass distribution for $H \rightarrow Z Z \rightarrow 4 e$ for $m_{H}=126 \mathrm{GeV}$ using ECAL-only electron momentum estimation (green open points: $\mathrm{ECAL}_{\mathrm{std}}$ only), and using the method employed in this analysis (black full points: $E_{\text {regr }}-p$ combination). The fitted standard deviation, $\sigma_{\mathrm{dCB}}$, of the double-sided Crystal-Ball [90] function and effective width $\sigma_{\text {eff }}$ defined in the text are indicated. Electrons with $p_{\mathrm{T}}^{e}>7 \mathrm{GeV}$ in the full $\eta^{e}$ range are used. (bottom) Expected effective momentum resolution $\sigma_{\text {eff }} / p$ for electrons in the EB as a function of the momentum for the ECAL-only, the tracker-only, and the combined estimates.

muons. Very low- $p_{\mathrm{T}}$ muons $\left(p_{\mathrm{T}}^{\mu} \lesssim 5 \mathrm{GeV}\right.$ ) may not have sufficient energy to penetrate the entire muon system and leave track segments in one or two stations of the muon system, where a station is composed of multiple detection planes between two iron layers. Tracks matched to such segments form so-called tracker muon objects. More details on muon reconstruction in CMS can be found in Ref. [91]. Both global and tracker muons are used in this analysis. 
The muons are selected among the reconstructed muon track candidates by applying minimal requirements on the track segments in both the muon system and inner tracker system and taking into account compatibility with small energy deposits in the calorimeters [81,91].

The $p_{\mathrm{T}}$ resolution for muons in the momentum range relevant for this analysis varies between $1.3 \%$ and $2.0 \%$ in the barrel, and up to $6 \%$ in the end caps. The dominant effect determining this resolution is the multiple scattering of muons in the tracker material. The achieved statistical accuracy on the determination of the position of the tracker modules is generally better than $10 \mu \mathrm{m}$, reaching a level of $\leq 2 \mu \mathrm{m}$ in the pixel tracker. Besides cosmic ray tracks, the usage of resonance mass and vertex information in the alignment procedure successfully constrains systematic deformations of the geometry that could bias reconstructed track parameters [92].

The accuracy of the hit measurements in the muon chambers and the overall alignment contribute to a lesser degree to the momentum measurement. This is achieved using several alignment procedures using cosmic muons, optical surveys, a laser system, and, finally, $Z \rightarrow \mu^{+} \mu^{-}$ events.

\section{Lepton isolation and vertex compatibility}

Lepton isolation is used to discriminate leptons originating from high- $p_{\mathrm{T}}$ boson decay, as in the case of the signal, from those arising from hadronic processes, which are typically immersed in a jet of other hadrons.

The isolation of individual leptons, measured relative to their transverse momentum $p_{\mathrm{T}}^{\ell}$, is defined by

$$
\begin{aligned}
R_{\mathrm{Iso}}^{\ell} \equiv & \left(\sum p_{\mathrm{T}}^{\text {charged }}+\max \left[0, \sum p_{\mathrm{T}}^{\text {neutral }}\right.\right. \\
& \left.\left.+\sum p_{\mathrm{T}}^{\gamma}-p_{\mathrm{T}}^{\mathrm{PU}}(\ell)\right]\right) / p_{\mathrm{T}}^{\ell},
\end{aligned}
$$

where the sums are over charged and neutral PF candidates in a cone $\Delta R=\sqrt{(\Delta \eta)^{2}+(\Delta \phi)^{2}}<0.4$ around the lepton direction at the interaction vertex, where $\Delta \eta=\eta^{\ell}-\eta^{i}$ and $\Delta \phi=\phi^{\ell}-\phi^{i}$ quantify the angular distance of the PF candidate $i$ from the lepton $\ell$ in the $\eta$ and $\phi$ directions, respectively. In Eq. (1), $\sum p_{\mathrm{T}}^{\text {charged }}$ is the scalar sum of the transverse momenta of charged hadrons originating from the chosen primary vertex of the event. The primary vertex is selected to be the one with the highest sum of $p_{T}^{2}$ of associated tracks. The sums $\sum p_{\mathrm{T}}^{\text {neutral }}$ and $\sum p_{\mathrm{T}}^{\gamma}$ are the scalar sums of the transverse momenta for neutral hadrons and photons, respectively. The latter excludes photons that are candidates for final-state radiation from the lepton, as defined in Sec. VI. The contribution from pileup $\left[p_{\mathrm{T}}^{\mathrm{PU}}(\ell)\right]$ in the isolation cone is subtracted from $R_{\text {Iso }}^{\ell}$ with different techniques for electrons and muons. For electrons, the FASTJET technique [93-95] is used, in which $p_{\mathrm{T}}^{\mathrm{PU}}(e) \equiv \rho \times A_{\text {eff }}$, where the effective area, $A_{\text {eff }}$, is the geometric area of the isolation cone scaled by a factor that accounts for the residual dependence of the average pileup deposition on the electron $\eta^{e}$. The variable $\rho$ is defined as the median of the energy-density distribution for the neutral particles within the area of any jet in the event, reconstructed using the $k_{\mathrm{T}}$ clustering algorithm $[96,97]$ with distance parameter $D=0.6$, with $p_{\mathrm{T}}^{\mathrm{jet}}>3 \mathrm{GeV}$ and $|\eta|<2.5$. For muons, $p_{\mathrm{T}}^{\mathrm{PU}}(\mu) \equiv 0.5 \times \sum_{i} p_{\mathrm{T}}^{\mathrm{PU}, i}$, where $i$ runs over the momenta of the charged hadron PF candidates not originating from the primary vertex. The factor 0.5 in the sum corrects for the different fraction of charged and neutral particles in the isolation cone. The electrons or muons are considered isolated if $R_{\mathrm{Iso}}^{\ell}<0.4$. The isolation requirement has been optimized to maximize the discovery potential in the full $m_{H}$ range of this analysis.

In order to suppress leptons originating from in-flight decays of hadrons and muons from cosmic rays, all leptons are required to come from the same primary vertex. This is achieved by requiring $\operatorname{SIP}_{3 \mathrm{D}}<4$, where $\operatorname{SIP}_{3 \mathrm{D}} \equiv$ $\mathrm{IP}_{3 \mathrm{D}} / \sigma_{\mathrm{IP}_{3 \mathrm{D}}}$ is the ratio of the impact parameter of the lepton track $\left(\mathrm{IP}_{3 \mathrm{D}}\right)$ in three dimensions $(3 \mathrm{D})$, with respect to the chosen primary vertex position, and its uncertainty.

\section{Lepton momentum scale, resolution and selection efficiency}

The determination of the momentum differs for electrons and muons, and it depends on the different CMS subdetectors involved in their reconstruction. The CMS simulation used in this analysis is based on the best knowledge of the detector conditions, as encoded in the ECAL calibrations and tracker and muon system alignment. Nevertheless, small discrepancies between data and simulation remain. In the case of the electron momentum scale and resolution, the main sources of discrepancy are the residual tracker misalignment and the imperfect corrections at the crystal level of the transparency loss due to irradiation, especially in the forward region. The average measured drop in energy response, before the crystal calibrations, is about 2\%-3\% in the barrel, rising to $20 \%$ in the range $2.1 \leq\left|\eta^{e}\right| \leq 2.5$ [37], and it is reduced to a subpercent level after the calibrations. In the case of muons, the momentum determination is affected by the tracker and muon system alignment geometry used for the reconstruction. The misalignment of the tracker causes a dependence of the systematic uncertainties in the reconstructed muon momentum on the $\eta^{\mu}, \phi^{\mu}$, and charge measurements.

The momentum scale and resolution for electrons and muons are studied using different data control samples for different $p_{\mathrm{T}}^{\ell}$ ranges. In the range of interest for this analysis $\left(p_{\mathrm{T}}^{\ell}<100 \mathrm{GeV}\right)$, the dileptons from decays of the $J / \psi$, $\Upsilon(\mathrm{nS})$, and $Z$ resonances are used to calibrate or validate the momentum scale and measure the momentum resolution. The $J / \psi$ and $\Upsilon(\mathrm{nS})$ decays constitute a clean data source of 
TABLE I. Number of $Z \rightarrow \ell^{+} \ell^{-}, J / \psi \rightarrow \ell^{+} \ell^{-}$and $\Upsilon(\mathrm{nS}) \rightarrow$ $\ell^{+} \ell^{-}$[sum of $\Upsilon(1 S), \Upsilon(2 S)$ and $\Upsilon(3 S)$ ] used to calibrate or validate lepton momentum scale and resolution and to measure lepton efficiencies $\left(Z \rightarrow \ell^{+} \ell^{-}\right.$only) in 7 and $8 \mathrm{TeV}$ data. Lowmass dimuon resonances are collected with specialized triggers.

\begin{tabular}{cccc}
\hline \hline$\ell$ & $Z \rightarrow \ell^{+} \ell^{-}$ & $J / \psi \rightarrow \ell^{+} \ell^{-}$ & $\Upsilon(\mathrm{nS}) \rightarrow \ell^{+} \ell^{-}$ \\
\hline$e$ & $10^{7}$ & $5 \times 10^{3}$ & $2.5 \times 10^{4}$ \\
$\mu$ & $1.4 \times 10^{7}$ & $2.7 \times 10^{7}$ & $1.5 \times 10^{7}$ \\
\hline \hline
\end{tabular}

low- $p_{\mathrm{T}}$ electrons and muons and are used to validate (calibrate) the electron (muon) momentum scale for $p_{\mathrm{T}}^{\ell}<20 \mathrm{GeV}$. The $Z \rightarrow \ell^{+} \ell^{-}$decay mode is a copious and pure source of leptons, with a wide momentum range covering the full spectrum of leptons of interest to this analysis. Table I provides the approximate number of dilepton resonance decays reconstructed in the 7 and $8 \mathrm{TeV}$ data used for the calibration of the lepton momentum.

For electrons, the calibration procedure consists of three steps. First, a set of corrections for the momentum scale is obtained by comparing the displacement of the peak position in the distributions of the $Z$-boson mass in the data and in the simulation in different $\eta$ regions and in two categories depending on the amount of bremsstrahlung. The corrections are derived as a function of time in order to account for the time-dependent crystal transparency loss [37]. Second, a linearity correction to the momentum scale is applied to account for the $p_{\mathrm{T}}$-dependent differences between data and simulation by comparing the dielectron mass distributions, binned in $p_{\mathrm{T}}^{e}$ of one of the two electrons, in data and in simulated $Z \rightarrow e^{+} e^{-}$events. The $J / \psi \rightarrow$ $e^{+} e^{-}$and $\Upsilon(1 S) \rightarrow e^{+} e^{-}$events are used as validation for electron $p_{\mathrm{T}}^{e}<20 \mathrm{GeV}$. All the corrections on the electron momentum scale from the first two steps are applied to data. Third, the energies of single electrons in the simulation are smeared by applying a random Gaussian multiplicative factor of mean 1 and width $\Delta \sigma$, in order to achieve the resolution observed in the data $Z$-boson sample.

For muons, an absolute measurement of momentum scale and resolution is performed by using a reference model of the $Z$ line shape convolved with a Gaussian function. The bias in the reconstructed muon $p_{\mathrm{T}}$ is determined from the position of the $Z$ mass peak as a function of muon kinematic variables, and a correction is derived for the data according to the procedure of Ref. [91]. A correction for the resolution is also derived for the simulation from a fit to the $Z \rightarrow \mu^{+} \mu^{-}$mass spectrum. The large event sample based on low-mass dimuon resonances provides an additional calibration source for the momentum resolution in a similar manner.

After this calibration, the lepton momentum scale and resolution are validated in data using dileptons from $J / \psi$, $\Upsilon(\mathrm{nS})$ and $Z$ decays in several bins of lepton $\eta^{\ell}$ and $p_{\mathrm{T}}^{\ell}$ in order to cover the full momentum range relevant for the $H \rightarrow Z Z \rightarrow 4 \ell$ search. Electrons with $p_{\mathrm{T}}^{e}>7 \mathrm{GeV}$ and muons with $p_{\mathrm{T}}^{\mu}>5 \mathrm{GeV}$ are considered. For the selection of $Z \rightarrow \ell^{+} \ell^{-}$events, all lepton selection criteria are applied as in the $H \rightarrow Z Z \rightarrow 4 \ell$ analysis.

The events are separated into categories according to the $p_{\mathrm{T}}^{e}$ and $\left|\eta^{e}\right|$ of one of the electrons, integrating over the other, while for dimuons, the average $p_{\mathrm{T}}^{\mu}$ and $\left|\eta^{\mu}\right|$ are used. The dilepton mass distributions in each category are fitted with a BW parameterization convolved with a single-sided Crystal-Ball (CB) function [90] [dimuon resonances or dielectron $J / \psi$ and $\Upsilon(1 S)]$ or with $\mathrm{MC}$ templates $(Z \rightarrow e e)$. From these fits, the offset in the measured peak position in data with respect to the nominal $Z$ mass, $\Delta m_{\text {data }}=m_{\text {data }}^{\text {peak }}-m_{Z}$, with respect to that found in the simulation, $\Delta m_{\mathrm{MC}}=m_{\mathrm{MC}}^{\text {peak }}-m_{Z}$, is extracted. Figure 2 shows the relative difference between data and simulation of the dilepton mass scale. After the electron calibration, the relative momentum scale between data and simulation is consistent within $0.2 \%$ in the central barrel and up to $\approx 0.3 \%$ in the forward part of the ECAL end caps. The residual dependence at low momentum is due to the use of wide bins in measured electron $p_{\mathrm{T}}^{e}$ in evaluating the $Z$-peak mass shift. The measured $p_{\mathrm{T}}^{e}$ dependence of the momentum scale before the $p_{\mathrm{T}}^{e}$ linearity correction, up to $0.6 \%$ in the central barrel and up to $1.5 \%$ in the end cap, is propagated to the reconstructed four-lepton mass from simulated Higgs boson events. The resulting shift of $0.3 \%(0.1 \%)$ for the $4 e$ $(2 e 2 \mu)$ channel is assigned as a systematic uncertainty in the signal mass scale. For muons, the agreement between the observed and simulated mass scales is within $0.1 \%$ in the entire pseudorapidity range of interest. A somewhat larger offset is seen for $J / \psi$ events with two high- $p_{\mathrm{T}}^{\mu}$ muons in the very forward region. However, for these events, the muons are nearly collinear, and such a kinematic configuration is very atypical for the $H \rightarrow Z Z \rightarrow 4 \ell$ events. Hence, the observed larger mass scale offset for such events is irrelevant in the context of this analysis.

Similarly, the widths of the peak due to instrumental resolution in data, $\sigma_{\text {data }}$, and in the simulation, $\sigma_{\mathrm{MC}}$, are compared. For electrons, $\sigma_{\text {eff }}$ ranges from $1.2 \%$ for the best category, which consists of two central single-cluster electrons with a small amount of bremsstrahlung ["barrel golden" (BG) [98]], to 4\% for the worst category, which consists of two electrons either with multiple clusters or with a high amount of bremsstrahlung, one central and one forward ["barrel showering" (BS) and "end-cap showering" (ES) [98]]. The amount of energy lost by bremsstrahlung before the electron reaches the ECAL is estimated with the GSF algorithm. The relative difference in $\sigma_{\text {eff }}$ between data and simulation is less than $3 \%$, for different electron categories [Fig. 3 (top)]. For the muons, in the whole kinematic range considered for this analysis, the instrumental Z-peak mass resolution observed in data is consistent with that in the simulation within about $5 \%$, when not considering $J / \psi$ events with two high- $p_{\mathrm{T}}^{\mu}$, high- $\left|\eta^{\mu}\right|$ muons [Fig. 3 (bottom)]. 

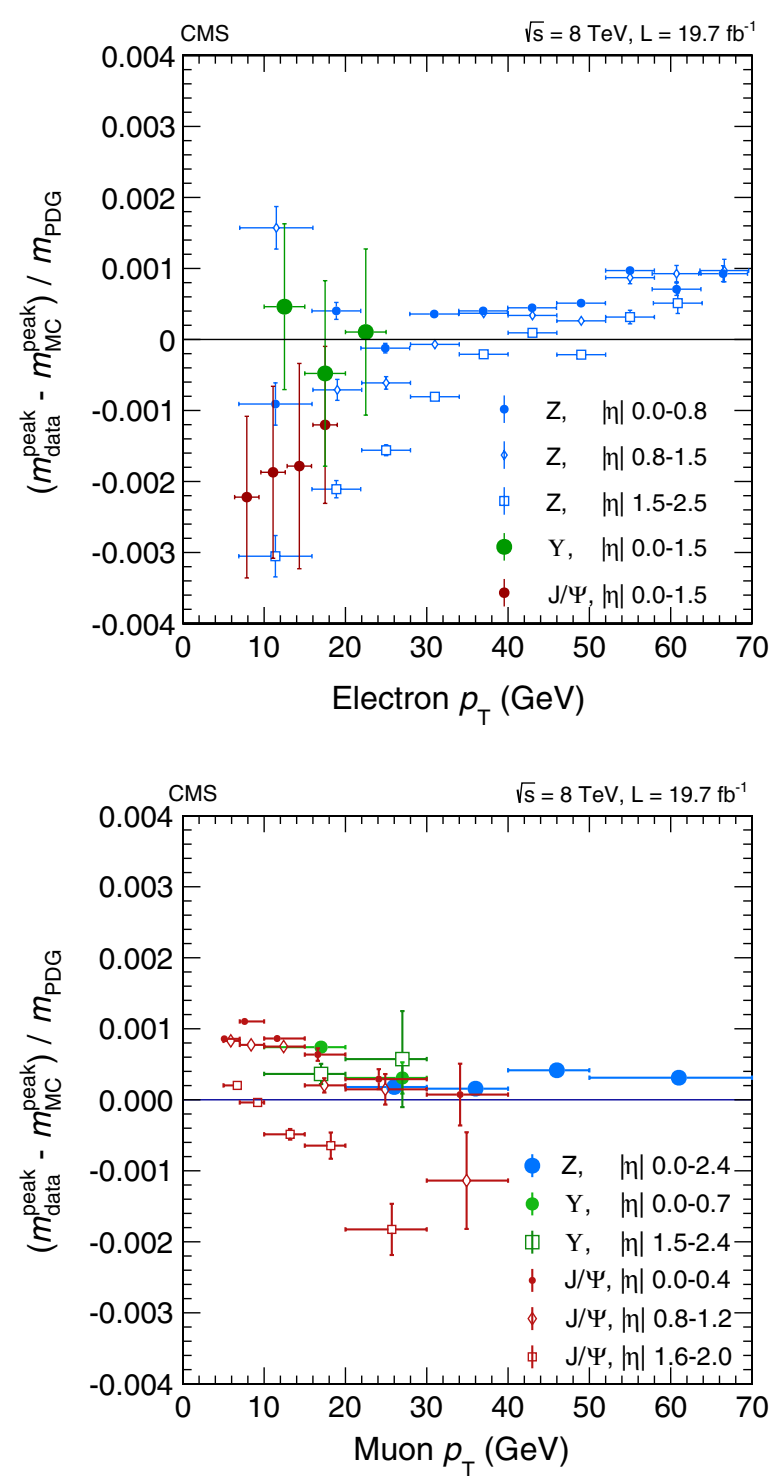

FIG. 2 (color online). Relative difference between the dilepton mass peak positions in data and simulation as obtained from $Z$, $J / \psi$ and $\Upsilon(\mathrm{nS})$ resonances as a function of (top) the transverse momentum of one of the electrons regardless of the second for dielectron events, and (bottom) the average muon $p_{\mathrm{T}}^{\mu}$ for dimuon events for the $8 \mathrm{TeV}$ data.

The combined efficiency for the reconstruction, identification, and isolation (and conversion rejection for electrons) of prompt electrons or muons is measured in data using a "tag and probe" method [99] based on an inclusive sample of $Z$-boson events, separately for 7 and $8 \mathrm{TeV}$ data. The efficiency is measured from the $Z \rightarrow \ell^{+} \ell^{-}$yields obtained by fitting the $Z$ line shape plus a background model to the dilepton mass distributions in two samples, the first with the probe lepton satisfying the selection criteria, and the second with the probe lepton failing them. The same approach is used in both data and simulation, and the ratio of the efficiency in the different $p_{\mathrm{T}}^{\ell}$ and $\eta^{\ell}$ bins of
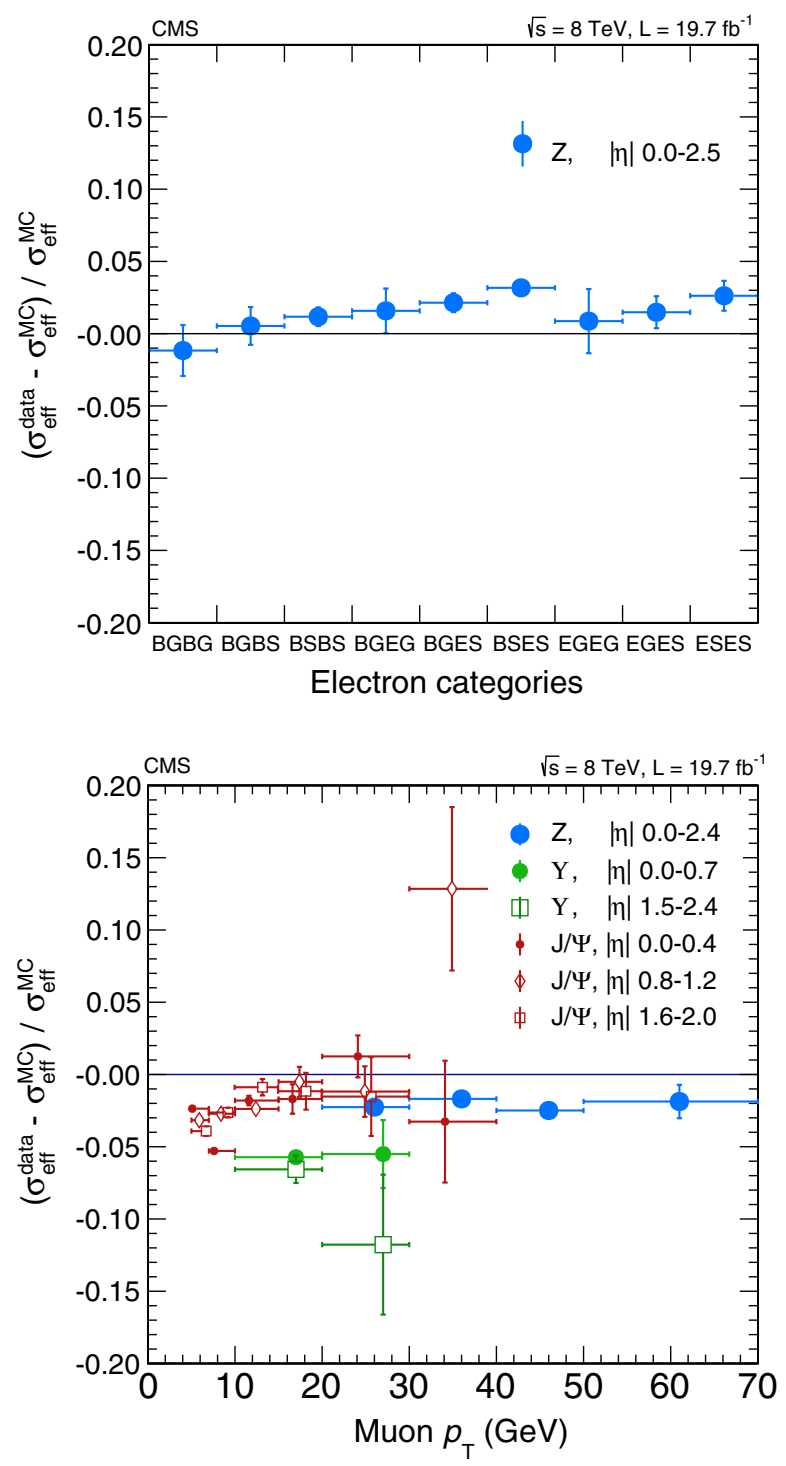

FIG. 3 (color online). (top) Relative difference between the dielectron $\sigma_{\text {eff }}$ in data and simulation, as measured from $Z \rightarrow e^{+} e^{-}$events, where the electrons are classified into different categories (B: barrel, E: end caps, G: golden, S: showering). (bottom) Relative difference between the dimuon mass resolutions in data and simulation as measured from $J / \psi, \Upsilon(\mathrm{nS})$, and $Z$ decays as functions of the average muon $p_{\mathrm{T}}^{\mu}$. The uncertainties shown are statistical only. Results are presented for data collected at $\sqrt{s}=8 \mathrm{TeV}$.

the probed lepton is used in the analysis to rescale the selection efficiency in the simulated samples. The efficiencies for reconstructing and selecting electrons and muons in the full $p_{\mathrm{T}}^{\ell}$ and $\eta^{\ell}$ range exploited in this analysis are shown in Fig. 4. The deviation of the efficiency in simulation relative to data, for the majority of the phase space of the leptons, is less than $3 \%$ for both electrons and muons. In the case of electrons with $p_{\mathrm{T}}^{e}<15 \mathrm{GeV}$, the deviation is larger, 5\%-9\%, but still consistent with unity, given the large statistical uncertainty. The dependency of the 

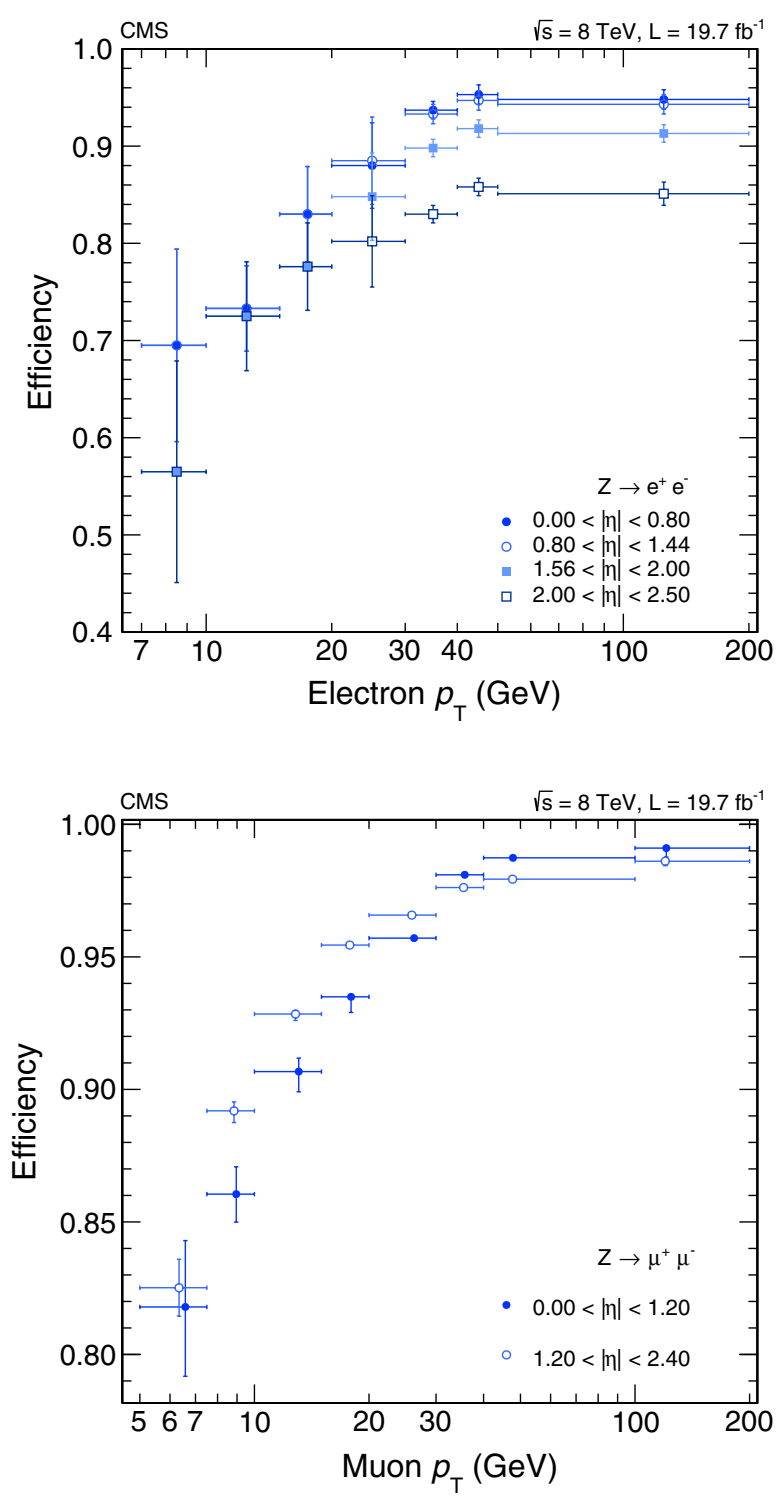

FIG. 4 (color online). Efficiency, as a function of the lepton $p_{\mathrm{T}}^{\ell}$, for reconstructing and selecting (top) electrons and (bottom) muons, measured with a $Z \rightarrow \ell \ell$ data sample by using a tag-andprobe method.

reconstruction and selection efficiency on the number of reconstructed primary vertices in the event is negligible for both the 7 and $8 \mathrm{TeV}$ data samples. The tracking efficiency decreases by about $0.4 \%$ between 1 and 21 pileup interactions, independent of the data-taking period.

\section{FINAL-STATE RADIATION RECOVERY}

A Z-boson decay into a lepton pair can be accompanied by final-state radiation, in which case it is desirable to identify and associate the radiated photon to the corresponding lepton to form the $Z$-boson candidate: $Z \rightarrow \ell^{+} \ell^{-} \gamma$. Photons reconstructed within $\left|\eta^{\gamma}\right|<2.4$ are possible FSR candidates. Low-energy photons are identified and reconstructed with the PF reconstruction with a dedicated clustering algorithm, efficient down to an energy of $230 \mathrm{MeV}$ in the EB and $600 \mathrm{MeV}$ in the EE [80]. The determination of the photon energies and directions is monitored in the data with $\pi^{0} \rightarrow \gamma \gamma$ decays, and is in agreement with the predictions from simulation.

Final-state radiated photons are mostly produced with a direction nearly collinear with the parent lepton and have a harder spectrum than background photons from initial-state radiation or pileup interactions. Therefore, to be identified as FSR, a reconstructed photon must either have a transverse momentum $p_{\mathrm{T}}^{\gamma}>2 \mathrm{GeV}$ and be found within a cone of size $\Delta R<0.07$ from a selected lepton candidate, or have $p_{\mathrm{T}}^{\gamma}>4 \mathrm{GeV}$ and be found isolated from charged particles and energy deposits and within $0.07<\Delta R<0.5$ from a selected lepton candidate.

The photon isolation observable $R_{\text {Iso }}^{\gamma}$ is the sum of the transverse momenta of charged hadrons, other photons, and neutral hadrons (including the ones originating from other vertices with respect to the primary vertex of the event) identified by the PF reconstruction within $\Delta R=0.3$ around the candidate photon direction, divided by the photon transverse momentum. Isolated photons must satisfy $R_{\text {Iso }}^{\gamma}<1$.

If more than one FSR candidate is associated with a $Z$ candidate, the one with the highest $p_{\mathrm{T}}^{\gamma}$ is chosen, if there is at least one with $p_{\mathrm{T}}^{\gamma}>4 \mathrm{GeV}$; otherwise, the one closest to any of the individual daughter leptons of the Z-boson candidate is chosen. These criteria are chosen to maximize the efficiency of the selection for photon emissions collinear with the lepton direction, while keeping the contribution from background or pileup interactions sufficiently low. The performance of the FSR recovery algorithm on the simulation of signal events is described in Sec. VIII.

\section{JET RECONSTRUCTION AND IDENTIFICATION}

In the analysis, the presence of jets is used as an indication of vector-boson fusion (VBF) or associated production with a weak boson, $V H$, with $V=W$ or $Z$, where the $V$ decays hadronically. Jets are reconstructed using the anti- $k_{\mathrm{T}}$ clustering algorithm [100] with distance parameter $D=0.5$, as implemented in the FASTJET package [95,101], applied to the PF candidates of the event. Jet energy corrections are applied as a function of the jet $p_{\mathrm{T}}^{\text {jet }}$ and $\eta^{\text {jet }}$ [102]. An offset correction is applied to subtract the energy contribution not associated with the high- $p_{\mathrm{T}}$ scattering, such as electronic noise and pileup, based on the jet-area method $[93,94,102]$. Jets are only considered if they have $p_{\mathrm{T}}^{\text {jet }}>30 \mathrm{GeV}$ and $\left|\eta^{\text {jet }}\right|<4$.7. In addition, they are required to be separated from the lepton candidates and from identified FSR photons by $\Delta R>0.5$.

Within the tracker acceptance, the jets are reconstructed with the constraint that the charged particles are compatible with the primary vertex. In addition, in the entire 
acceptance, a multivariate discriminator is used to separate jets arising from the primary interaction from those reconstructed from energy deposits associated with pileup interactions, especially due to neutral particles not associated with the primary vertex of the event. The discrimination is based on the differences in the jet shapes, the relative multiplicity of charged and neutral components, and the fraction of transverse momentum carried by the hardest components [103].

\section{SELECTION AND CATEGORIZATION OF FOUR-LEPTON CANDIDATES}

The event selection is designed to give a set of signal candidates in the $H \rightarrow Z Z \rightarrow 4 \ell$ final state in three mutually exclusive subchannels: $4 e, 2 e 2 \mu$, and $4 \mu$. Four well-identified and isolated leptons are required to originate from the primary vertex to suppress the $Z+$ jet and $t \bar{t}$ backgrounds.

A $Z$ candidate formed with a pair of leptons of the same flavor and opposite charge $\left(\ell^{+} \ell^{-}\right)$is required. When forming the $Z$-boson candidates, only FSR photon candidates that make the lepton-pair mass closer to the nominal $Z$-boson mass are incorporated. If the mass $m_{\ell \ell \gamma}>$ $100 \mathrm{GeV}$, the photon is not considered, to minimize the fraction of misidentified FSR candidates. With the photon selection requirements described in Sec. VI, about $1.5 \%$, $4.6 \%$, and $9 \%$ of the simulated $H \rightarrow 4 e, H \rightarrow 2 e 2 \mu$, and $H \rightarrow 4 \mu$ decays, respectively, are affected by the photon recovery procedure. As the photon emission is most often collinear with one of the leptons, measured electron energies, by construction, include the energy of a large fraction of the emitted photons in the associated ECAL supercluster, while measured muon momenta do not include the emitted photons. Therefore, without photon recovery, FSR is expected to degrade the four-lepton mass resolution for Higgs boson candidates, especially in the $4 \mu$ and in the $2 e 2 \mu$ final states and, to a lesser extent, in the $4 e$ final state. The performance of the FSR recovery algorithm is estimated using simulated samples of $H \rightarrow Z Z \rightarrow 4 \ell$, and the rate is verified with inclusive $Z$ and $Z Z$ data events. Genuine FSR photons within the acceptance of the FSR selection are selected with an efficiency of $\approx 50 \%$ and with a mean purity of $80 \%$. The FSR photons are selected in $5 \%$ of inclusive $Z$ events with muon pairs, and in $0.5 \%$ of single- $Z$ events with electron pairs. A gain of $\approx 3 \%$ ( $2 \%$, $1 \%)$ in efficiency is expected for the selection of $H \rightarrow 4 \mu$ $(2 e 2 \mu, 4 e)$ events in this analysis. The momentum of the selected FSR photon is added to the momentum of the nearest lepton for the computation of every $4 \ell$ kinematic variable. Hereafter, $\ell$ denotes a $\ell+\gamma$, in the case of a recovered FSR photon.

Among all the possible opposite-charge lepton pairs in the event, the one with an invariant mass closest to the nominal $Z$-boson mass is denoted $Z_{1}$ and retained if its mass, $m_{Z_{1}}$, satisfies $40<m_{Z_{1}}<120 \mathrm{GeV}$. Then, all remaining leptons are considered and a second $\ell^{+} \ell^{-}$pair is required $\left(Z_{2}\right)$, with the mass denoted $m_{Z_{2}}$. If more than one $Z_{2}$ candidate is selected, the ambiguity is resolved by choosing the pair of leptons with the highest scalar sum of $p_{\mathrm{T}}$. Simulation studies demonstrate that this algorithm selects the true $Z_{2}$ in the majority of cases without sculpting the shape of the $Z Z$ background. The chosen $Z_{2}$ is required to satisfy $12<m_{Z_{2}}<120 \mathrm{GeV}$. For the mass range of $m_{H}<180 \mathrm{GeV}$, at least one of the $Z$ candidates is off shell. The lower bound for $m_{Z_{2}}$ provides an optimal sensitivity for a Higgs boson mass hypothesis in the range $110<m_{H}<160 \mathrm{GeV}$.

Among the four selected leptons forming the $Z_{1}$ and the $Z_{2}$, at least one lepton is required to have $p_{\mathrm{T}}^{\ell}>20 \mathrm{GeV}$, and another one is required to have $p_{\mathrm{T}}^{\ell}>10 \mathrm{GeV}$. These $p_{\mathrm{T}}^{\ell}$ thresholds ensure that the selected events have leptons on the efficiency plateau of the trigger. To further remove events with leptons originating from hadron decays produced by jet fragmentation or from the decay of low-mass hadron resonances, it is required that any opposite-charge pair of leptons chosen among the four selected leptons (irrespective of flavor) satisfy $m_{\ell^{+} \ell^{-}}>4 \mathrm{GeV}$. The phase space for the search of the SM Higgs boson is defined by restricting the measured mass range to $m_{4 \ell}>100 \mathrm{GeV}$.

The overall signal detection efficiencies, including geometrical acceptance, for the $4 e, 2 e 2 \mu$, and $4 \mu$ channels increase as a function of $m_{H}$ rapidly up to approximately $2 m_{Z}$, where both the $Z$ bosons are on shell, and then flattens. The residual rise for $m_{H}>300 \mathrm{GeV}$ is mostly due to the increased acceptance. The efficiency versus $m_{H}$ is shown in Fig. 5 for the gluon fusion Higgs boson production mode, and it is very similar for other production modes. The signal events are generated with $\left|\eta^{\ell}\right|<5$ and invariant mass of the dileptons from both the $Z_{1}$ and the $Z_{2}$

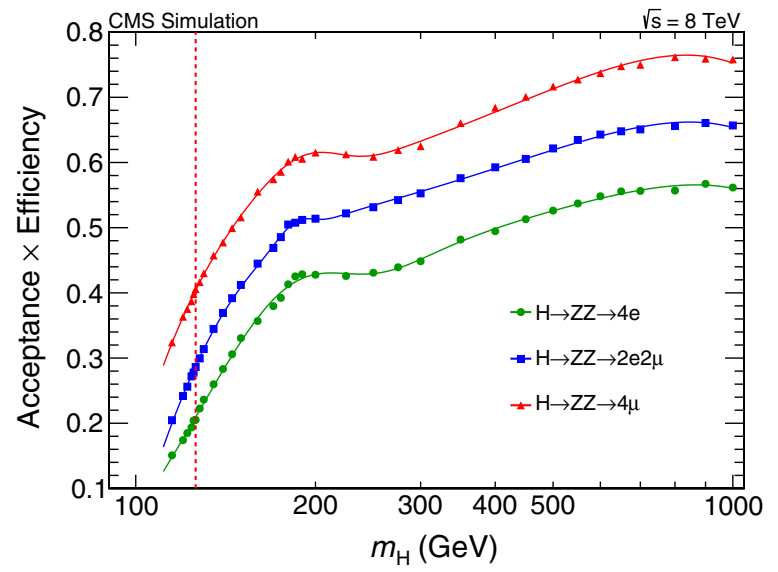

FIG. 5 (color online). Geometrical acceptance times selection efficiency for the SM Higgs boson signal as a function of $m_{H}$ in the three final states for gluon fusion production. Points represent efficiency estimated from full CMS simulation; lines represent a smooth polynomial curve interpolating the points, used in the analysis. The vertical dashed line represents $m_{H}=126 \mathrm{GeV}$. 
boson decays $m_{\ell^{+} \ell^{-}}>1 \mathrm{GeV}$. The efficiency within the geometrical acceptance is $\approx 30 \% \quad(58 \%), 43 \% \quad(71 \%)$, and $62 \%(87 \%)$ for the three channels, respectively, for $m_{H}=126(200) \mathrm{GeV}$.

For a Higgs boson with $m_{H}=126 \mathrm{GeV}$, the resolution of the Gaussian core of the mass distribution, estimated from simulated signal samples with a double-sided CrystalBall function fit, is about 2.0, 1.6, $1.2 \mathrm{GeV}$ for $4 e, 2 e 2 \mu$, and $4 \mu$, respectively. The full $\mathrm{rms}$ of the four-lepton mass distribution, including the asymmetric tails, is estimated to be $2.9,2.3,1.7 \mathrm{GeV}$ for the three channels, respectively. For a Higgs boson with $m_{H}=600 \mathrm{GeV}$, in which the natural width of the resonance contributes most, the double-sided Crystal-Ball-function core width parameter is about $75 \mathrm{GeV}$.

While in the dominant gluon fusion mechanism the Higgs boson is produced only in association with jets from initial-state radiation of the quarks, in the VBF production the two vector bosons are radiated from the initial-state quarks to produce the Higgs boson. The cross section for VBF production is about 1 order of magnitude smaller than that for the gluon fusion process. In the vector-boson scattering process, the two initial-state quarks deviate at a polar angle large enough such that as final-state quarks they create measurable additional jets in the event. These two jets, being remnants of the incoming proton beams, have typically a large separation in $\eta$ and high momentum. These characteristics are used to distinguish gluon fusion from VBF Higgs boson production in the analysis. Jets in the final state also come from $t \bar{t} H$ and $\mathrm{VH}$ production, where the $V$ decays hadronically.

In order to improve the sensitivity to the Higgs boson production mechanisms, the event sample is split into two categories based on the jet multiplicity, where a jet is defined as in Sec. VII. These categories are defined as the 0/1-jet category, containing events with fewer than two jets, and the dijet category, containing events with at least two jets. In the 0/1-jet category, the transverse momentum of the four-lepton system $\left(p_{\mathrm{T}}^{4 \ell}\right)$ is used to distinguish VBF production and associated production with a weak boson, $V H$, from gluon fusion. In the dijet category, a linear discriminant $\left(\mathscr{D}_{\text {jet }}\right)$ is formed combining two VBF-sensitive variables, the absolute difference in pseudorapidity $\left(\left|\Delta \eta_{j j}\right|\right)$ and the invariant mass of the two leading jets $\left(m_{j j}\right)$. The discriminant maximizes the separation between vectorboson and gluon fusion processes. In the 0/1-jet (dijet) category, about $5 \%(20 \%)$ of the signal events are expected to come from the VBF production mechanism, as estimated from simulation. The expected signal yield, split by category and by production mode, is reported in Table V.

\section{A. Per-event mass uncertainties}

For the Higgs boson mass and width measurement, the uncertainty in the four-lepton mass, which can be estimated on a per-event basis, is relevant because it varies considerably over the small number of selected events.

Uncertainties in the measured lepton momentum arise from imperfect calibration of the ECAL supercluster and uncertainty in the GSF track fit due to possible highbremsstrahlung emissions in the case of the electrons, and from the uncertainty in the muon track fit due to the multiple scattering of the muons in the material of the inner tracker. These uncertainties depend on and are evaluated from the lepton's direction and transverse momentum, as well as from possible mismeasurements specific to each lepton. In the case of electrons, the momentum uncertainties are assessed from the combination of the quality of the ECAL supercluster and the GSF track fit, through a similar multivariate regression as the one used to refine the estimate of the electron momentum, described in Sec. VA. In the case of muons, the momentum uncertainties are assessed from the properties of hits in the tracker and in the muon system, and the quality of the muon candidate fit. If FSR photons are identified and associated with the event, their uncertainty, assessed by the quality of the ECAL clusters, is also accounted for in the event mass uncertainty.

The momentum uncertainties for each of the four leptons in an event are then propagated into a relative uncertainty $\mathscr{D}_{\mathrm{m}} \equiv \sigma_{m_{4 \ell}} / m_{4 \ell}$ in the four-lepton mass. The per-event mass uncertainty is given as the sum in quadrature of the individual mass uncertainty contributions from each lepton and any identified FSR photon candidate. A calibration of the per-lepton uncertainties is derived using large $J / \psi \rightarrow \mu^{+} \mu^{-}, Z \rightarrow \mu^{+} \mu^{-}$, and $Z \rightarrow e^{+} e^{-}$event samples, both in data (Table I) and in simulation. The line shape of these resonances is modeled, as for the SM Higgs boson, with a BW convolved with a double-sided $\mathrm{CB}$ function, where the resolution is estimated as $\lambda \times \sigma\left(m_{4 \ell}\right)$. In this procedure, $\sigma\left(m_{4 \ell}\right)$ is fixed to the value computed using the uncertainties in the individual momenta of the leptons, and $\lambda$, defined as the calibration constant, is a floating parameter. The latter is derived for electrons and muons in several bins of the average $p_{\mathrm{T}}^{\ell}$ and $\eta^{\ell}$ of the lepton: $J / \psi \rightarrow \mu^{+} \mu^{-}$is used for muons with $p_{\mathrm{T}}^{\mu}<20 \mathrm{GeV}$, while, for lack of a sufficiently large sample of $J / \psi \rightarrow e^{+} e^{-}, Z \rightarrow e^{+} e^{-}$ events are used in the entire $p_{\mathrm{T}}^{e}$ range. The value of $\lambda$ obtained from the fit is approximately 1.2 for electrons and 1.1 for muons, in the entire kinematic range of the leptons used in this analysis.

As a closure test, the $Z \rightarrow \ell \ell$ events are grouped into subsets based on their per-event predicted dilepton mass resolution and fit to the $Z$ line shape in each subset as described above. A systematic uncertainty of $\pm 20 \%$ is assigned to the per-event mass uncertainty for both electrons and muons based on the agreement between per-event computed and observed mass resolutions as shown in Fig. 6 (top). In Fig. 6 (bottom), the comparison between data and simulation of the $\mathscr{D}_{\mathrm{m}}$ observable in the $Z \rightarrow 4 \ell$ mass region is shown. 

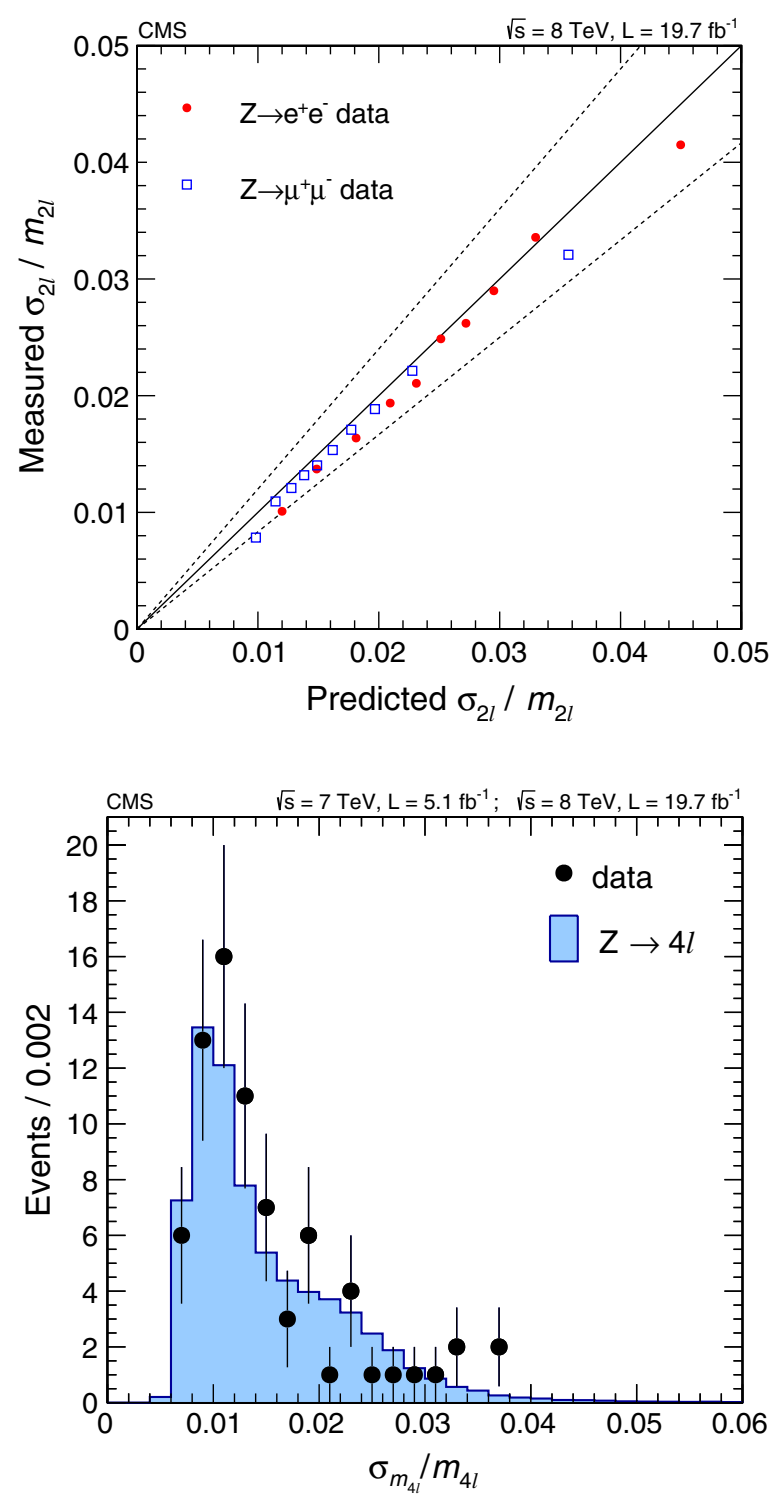

FIG. 6 (color online). (top) Measured versus predicted relative mass uncertainties for $Z \rightarrow e^{+} e^{-}$and $Z \rightarrow \mu^{+} \mu^{-}$events in data. The dashed lines represent the $\pm 20 \%$ envelope, used as systematic uncertainty in the resolution. (bottom) Relative mass uncertainty distribution for data and simulation in the $Z \rightarrow 4 \ell$ mass region of $80<m_{4 \ell}<100 \mathrm{GeV}$.

\section{BACKGROUND ESTIMATION}

The dominant background contribution in the $H \rightarrow$ $Z Z \rightarrow 4 \ell$ search is irreducible and is due to direct $Z Z$ production via $q \bar{q}$ annihilation and gluon fusion. The remaining subleading contributions arise from reducible multilepton sources, $Z+$ jets, $t \bar{t}$, and $W Z+$ jets.

\section{A. Irreducible background}

The expected yield and shape of the $Z Z$ background is evaluated by simulation. The NLO cross section for $q \bar{q} \rightarrow$ $Z Z$ production and the $\mathrm{LO}$ cross section for $g g \rightarrow Z Z$ production are calculated with MCFM [104-106]. The relative contribution of $\mathrm{LO} g g \rightarrow Z Z$ with respect to NLO $q \bar{q} \rightarrow Z Z$ is about $2 \%$ at four-lepton mass $m_{4 \ell}=$ $126 \mathrm{GeV}$ and about $6 \%$ at $1 \mathrm{TeV}$. The expected contribution of the $Z Z$ processes to the total background, in the region $100<m_{4 \ell}<1000\left(121.5<m_{4 \ell}<130.5\right) \mathrm{GeV}$, is approximately $91 \%, 94 \%$, and $97 \%(58 \%, 71 \%$, and $86 \%)$ in the $4 e, 2 e 2 \mu$, and $4 \mu$ channels, respectively. The shape uncertainties arising from imperfect simulation of the $p_{\mathrm{T}}^{\ell}$ and $\eta^{\ell}$ dependence of the efficiency and other experimental sources are completely overshadowed by the uncertainties from the normalization systematics, such that shape variations have negligible effects compared to the normalization variations.

The irreducible four-lepton background arising from double-parton interactions (DPI), Z + Drell-Yan (DY), is evaluated using PYTHIA 6.4.24 with the overall cross section calculated as $\sigma_{\mathrm{DPI}}=\sigma_{Z} \cdot \sigma_{\mathrm{DY}} / \sigma_{\text {pheno, }}$, where the phenomenological effective cross section, measured at $\sqrt{s}=7 \mathrm{TeV}$, is $\sigma_{\text {pheno }}=15 \mathrm{mb}$ [107], and the cross sections $\sigma_{Z}$ and $\sigma_{\mathrm{DY}}$ are taken from simulation. The DPI $Z+\mathrm{DY}$ background is much smaller than normalization uncertainties on either $q \bar{q} \rightarrow Z Z, g g \rightarrow Z Z$ or a reducible background; hence, the DPI $Z+D Y$ background is neglected in the analysis.

\section{B. Reducible background}

Two independent methods, using dedicated control regions in data, are considered to estimate the reducible background, denoted as $Z+X$ in the following paragraphs because the background is dominated by the $Z+$ jets process. The control regions are defined by a dilepton pair satisfying all the requirements of a $Z_{1}$ candidate and two additional leptons, opposite sign (OS) or same sign (SS), satisfying certain relaxed identification requirements when compared to those used in the analysis. The invariant mass of the additional dilepton pair is required to be larger than $12 \mathrm{GeV}$, in order to be consistent with the criteria imposed on the $Z_{2}$ candidate in the signal selection.

In both methods, the extrapolation from the control region to the signal region is performed using the lepton misidentification probability, $f\left(\ell, p_{\mathrm{T}}^{\ell},\left|\eta^{\ell}\right|\right)$, which is defined as the fraction of nonsignal leptons identified with the analysis selection criteria, estimated in an enriched sample of nongenuine electrons and muons. This sample is composed of $Z_{1}+1 \ell_{\text {loose }}$ events in data consisting of a pair of leptons, both passing the selection requirements used in the analysis, and exactly one additional lepton passing the relaxed selection. The mass of the $Z_{1}$ candidate is required to satisfy $\left|m_{\ell \ell}-m_{Z}\right|<10 \mathrm{GeV}$ for the OS leptons method. Such a stringent requirement suppresses from the $f\left(\ell, p_{\mathrm{T}}^{\ell},\left|\eta^{\ell}\right|\right)$ calculation the contribution of events with FSR where the photon converts and one of the conversion products is not reconstructed. For the SS leptons method, a requirement of $\left|m_{\ell \ell}-m_{Z}\right|<40 \mathrm{GeV}$ is 
imposed. In order to suppress the contribution from $W Z$ and $t \bar{t}$ processes, which have a third lepton, the missing transverse energy $\left(E_{\mathrm{T}}\right)$ is required to be less than $25 \mathrm{GeV}$. The $E_{\mathrm{T}}$ is defined as the modulus of the vector sum of the transverse momenta of all reconstructed PF candidates (charged or neutral) in the event. The invariant mass of the loose lepton and the opposite-sign lepton from the $Z_{1}$ candidate, if they have the same flavor, are required to be greater than $4 \mathrm{GeV}$ to reject contributions from low-mass resonances, such as $J / \psi$. As a result of these requirements, the control sample largely consists of events with a $Z$ boson and a misidentified additional lepton. Hence, the fraction of these events in which the additional lepton passes the analysis identification and isolation requirements gives a rate $f\left(\ell, p_{\mathrm{T}}^{\ell},\left|\eta^{\ell}\right|\right)$ that ranges from $1 \%-15 \%(5 \%-10 \%)$ depending on the $p_{\mathrm{T}}^{\ell}$ and $\eta^{\ell}$ of the electron (muon).

\section{Method using opposite-sign (OS) leptons}

In this method, the control region consists of events with a $Z_{1}$ candidate and two additional leptons with the same flavor and opposite charge. Two categories of events are considered in this method.

The category $2 \mathrm{P} 2 \mathrm{~F}$ is composed of events in which two leptons pass $(\mathrm{P})$ the selection requirements of the analysis and two fail $(\mathrm{F})$, but pass the loose selection. It is used to estimate the contribution from backgrounds that intrinsically have only two prompt leptons $(Z+$ jets, $t \bar{t})$. To estimate the contribution of these background processes in the signal region, each $2 \mathrm{P} 2 \mathrm{~F}$ event $i$ is weighted by a factor $\frac{f_{3}^{i}}{1-f_{3}^{i}} \frac{f_{4}^{i}}{1-f_{4}^{i}}$, where $f_{3}^{i}$ and $f_{4}^{i}$ are the $f\left(\ell, p_{\mathrm{T}}^{\ell},\left|\eta^{\ell}\right|\right)$ for the third and fourth lepton. Analogously, the $3 \mathrm{P} 1 \mathrm{~F}$ category consists of events where exactly one of the two additional leptons passes the analysis selection. It is used to estimate the contribution from backgrounds with three prompt leptons and one misidentified lepton $(W Z+$ jets and $Z \gamma+$ jets with the photon converting to an $e^{+} e^{-}$pair). Each event $j$ in the $3 \mathrm{P} 1 \mathrm{~F}$ control region is weighted by a factor $\frac{f_{a}^{j}}{1-f_{a}^{3}}$, where $f_{a}^{j}$ is the $f\left(\ell, p_{\mathrm{T}}^{\ell},\left|\eta^{\ell}\right|\right)$ for the third or fourth lepton to fail the analysis selection. This control region also has contributions from $Z Z$ events where one of the four prompt leptons fails the analysis selection, and from the processes with only two prompt leptons ( $2 \mathrm{P} 2 \mathrm{~F}$ type), where one of the two nonprompt leptons passes the selection requirements. The contribution from $Z Z$ events, $n_{3 \mathrm{PIF}}^{Z Z}$, is estimated from simulation, and the background estimate is reduced by a factor of $1-n_{3 \mathrm{PIF}}^{\mathrm{ZZ}} / N_{3 \mathrm{PIF}}$, where $N_{3 \mathrm{PIF}}$ is the number of events of the $3 \mathrm{P} 1 \mathrm{~F}$ control region. The contribution from $2 \mathrm{P} 2 \mathrm{~F}$-type processes in the $3 \mathrm{P} 1 \mathrm{~F}$ region is estimated as $\sum_{i}\left(\frac{f_{3}^{j}}{1-f_{3}^{i}}+\frac{f_{4}^{j}}{1-f_{4}^{i}}\right)$. It contributes to the final weighted sum of the $3 \mathrm{P} 1 \mathrm{~F}$ events with the component $\sum_{i}\left(2 \frac{f_{3}^{i}}{1-f_{3}^{i}} \frac{f_{4}^{i}}{1-f_{4}^{i}}\right)$, which has to be subtracted from the background estimate. Therefore, in this method, the expected yield for the reducible background in the signal region, $N_{\mathrm{SR}}^{\text {reducible }}$, becomes

$N_{\mathrm{SR}}^{\mathrm{reducible}}=\left(1-\frac{n_{3 \mathrm{P} 1 \mathrm{~F}}^{\mathrm{ZZ}}}{N_{3 \mathrm{P} 1 \mathrm{~F}}}\right) \sum_{j}^{N_{3 \mathrm{P} 1 \mathrm{~F}}} \frac{f_{a}^{j}}{1-f_{a}^{j}}-\sum_{i}^{N_{2 \mathrm{P} 2 \mathrm{~F}}} \frac{f_{3}^{i}}{1-f_{3}^{i}} \frac{f_{4}^{i}}{1-f_{4}^{i}}$.

\section{Method using same-sign (SS) leptons}

In this method, the control region consists of events with a $Z_{1}$ candidate and two additional leptons with the same flavor and same charge. The $f\left(\ell, p_{\mathrm{T}}^{\ell},\left|\eta^{\ell}\right|\right)$ is measured using a $Z_{1}+1 \ell_{\text {loose }}$ sample, which is similar to that used for the $O S$ control region, but with the invariant mass of the $Z_{1}$ candidate, $\left|m_{\ell \ell}-m_{Z}\right|<40 \mathrm{GeV}$, consistent with the requirement on the $Z_{1}$ candidate used in the analysis. Here, the contribution from FSR photons to the electron misidentification probability is much larger and needs to be taken into account. This is done by exploiting the observed linear dependence of the $f\left(e, p_{\mathrm{T}}^{e},\left|\eta^{e}\right|\right)$ on the fraction of loose electrons with tracks having one missing hit in the pixel detector, $r_{\text {miss }}\left(p_{\mathrm{T}}^{e},\left|\eta^{e}\right|\right)$, which is indicative of a possible conversion. The fraction $r_{\text {miss }}\left(p_{\mathrm{T}}^{e},\left|\eta^{e}\right|\right)$ is estimated using samples with different FSR contributions obtained by varying the requirements on $\left|m_{\ell \ell}-m_{Z}\right|$ and $\left|m_{\ell \ell e_{\text {loose }}}-m_{Z}\right|$. The corrected $\tilde{f}\left(e, p_{\mathrm{T}}^{e},\left|\eta^{e}\right|\right)$ is then computed using the value $r_{\text {miss }}\left(p_{\mathrm{T}}^{e},\left|\eta^{e}\right|\right)$ measured in the control sample where the method is applied.

The expected number of reducible background events in the signal region is obtained as

$$
N_{\mathrm{SR}}^{\text {reducible }}=r_{\mathrm{OS} / \mathrm{SS}} \cdot \sum_{i}^{N_{2 \mathrm{PL} L \mathrm{SS}}} \tilde{f}_{3}^{i} \cdot \tilde{f}_{4}^{i},
$$

where $N_{2 \mathrm{P}_{2} \mathrm{~L}_{\mathrm{SS}}}$ is the number of observed events in the region $2 \mathrm{P} 2 \mathrm{~L}_{\mathrm{SS}}$, in which both the additional leptons fulfill the loose selection requirements for leptons, having the same flavor and charge. The ratio $r_{\mathrm{O} / \mathrm{ss}}$ between the number of events in the $2 \mathrm{P} 2 \mathrm{~L}_{\mathrm{OS}}$ and $2 \mathrm{P} 2 \mathrm{~L}_{\mathrm{SS}}$ control regions is obtained from simulation.

\section{Combination of the two methods}

The predicted yields of the $Z+X$ background from the two methods are in agreement within their statistical uncertainties. The dominant sources of these uncertainties are the limited number of events in the $3 \mathrm{P} 1 \mathrm{~F}, 2 \mathrm{P} 2 \mathrm{~F}$, and $2 \mathrm{P} 2 \mathrm{~L}_{\mathrm{SS}}$ control regions, as well as in the region where the correction factor for $\tilde{f}\left(e, p_{\mathrm{T}}^{e},\left|\eta^{e}\right|\right)$ is computed. Since they are mutually independent, results of the two methods are combined.

The shape of the $m_{4 \ell}$ distribution for the reducible background is obtained from the OS method by fitting the $m_{4 \ell}$ distributions of $2 \mathrm{P} 2 \mathrm{~F}$ and $3 \mathrm{P} 1 \mathrm{~F}$ events separately with empirical functional forms built from Landau [108] and 

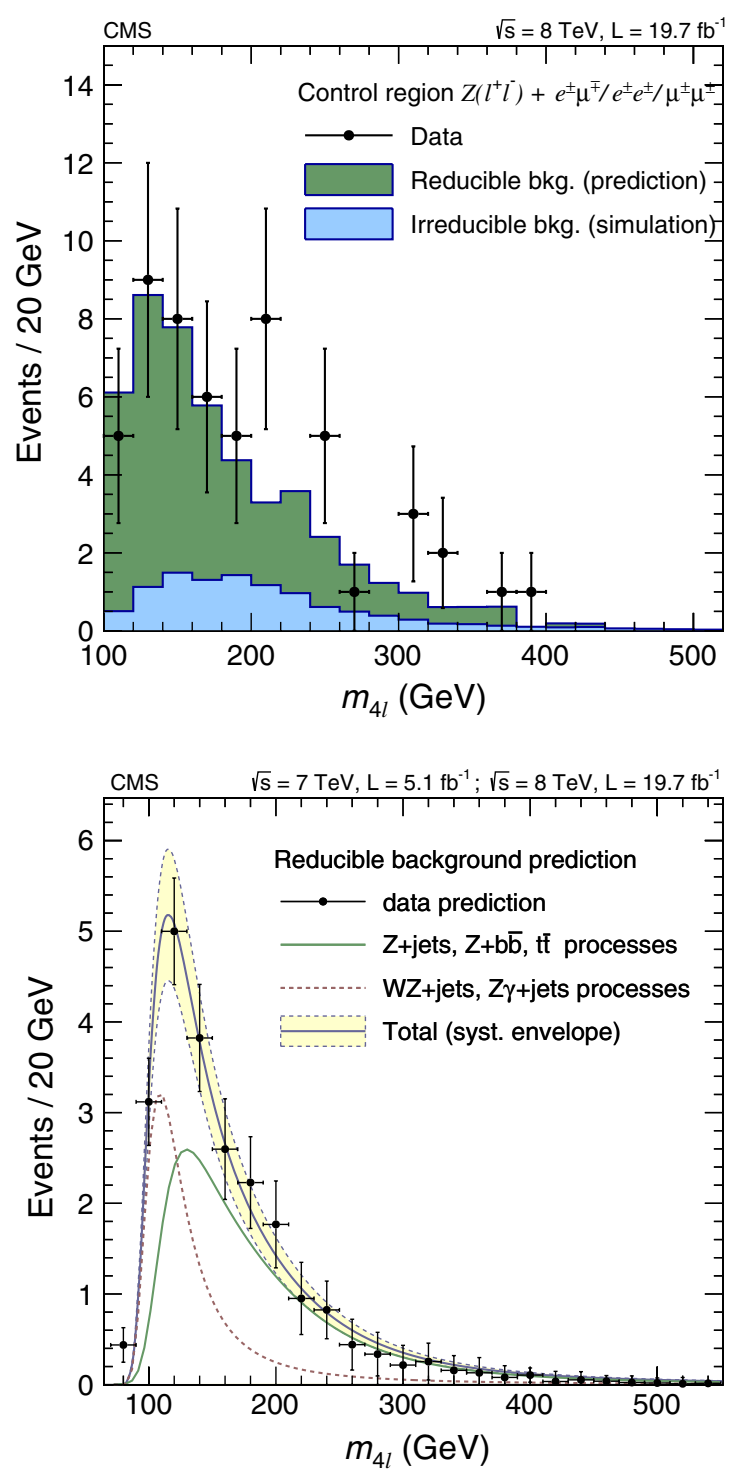

FIG. 7 (color online). (top) Validation of the method using the SS control sample. The observed $m_{4 \ell}$ distribution (black dots), prediction of the reducible background (dark green area), and expected contributions from $Z Z$ (light blue area) are shown. (bottom) Prediction for the reducible background in all three channels together (black dots), fitted using an empirical shape (blue curve) with indicated total uncertainty (yellow band). The contributions from the 2P2F-like (solid green) and 3P1F-like (dashed red) processes are fitted separately.

exponential distributions. The systematic uncertainty in the $m_{4 \ell}$ shape is determined by the envelope that covers alternative functional forms or alternative binning for the fit used to determine its parameters. The additional discriminating variables for this background are described by binned templates, as discussed in Sec. XII.

The total systematic uncertainties assigned to the $Z+X$ background estimate take into account the uncertainty in the $m_{4 \ell}$ shape. They also account for the difference in the composition of the $Z_{1}+1 \ell_{\text {loose }}$ sample used to compute $f\left(\ell, p_{\mathrm{T}}^{\ell},\left|\eta^{\ell}\right|\right)$ and the control regions in the two methods used to estimate the $Z+X$ background-in particular, the contribution of the heavy flavor jets and photon conversions. The systematic uncertainty is estimated to be $20 \%$, $25 \%$, and $40 \%$ for the $4 e, 2 e 2 \mu$, and $4 \mu$ decay channels, respectively. The two methods have been further validated using events that pass the analysis selection with the exception that the $Z_{2}$ candidate is formed out of a lepton pair with the wrong combination of flavors or charges (control region $Z_{1}+e^{ \pm} e^{ \pm} / e^{ \pm} \mu^{\mp} / \mu^{ \pm} \mu^{ \pm}$). The predicted contribution of the reducible background in this control region is in agreement with the observed number of events within the uncertainties. Figure 7 (top) shows the validation of the OS method.

The prediction for the $Z+X$ background yields with combined statistical and systematic uncertainties is given in Sec. XI and also shown in Fig. 7 (bottom). The expected yields of the $Z+X$ background in the signal region from the $2 \mathrm{P} 2 \mathrm{~F}$-like and $3 \mathrm{P} 1 \mathrm{~F}-\mathrm{like}$ sources are estimated separately. The weighted events of the two control regions are also fitted independently and then added together to give the total $Z+X m_{4 \ell}$ probability density function used in the fit. The relative contribution of the reducible background to the total background in the region $100<m_{4 \ell}<1000$ $\left(121.5<m_{4 \ell}<130.5\right) \mathrm{GeV}$ depends on the final state, being approximately $9 \%(42 \%), 6 \%(28 \%)$, and $3 \%(14 \%)$ in the $4 e, 2 e 2 \mu$, and $4 \mu$ channels, respectively. The estimated yields of this background are reported in Sec. XI.

\section{KINEMATIC DISCRIMINANTS}

The four-lepton decay mode has the advantage that the kinematics of the Higgs boson and its decay products are all visible in the detector, providing many independent observables that can be used for different purposes. First, in addition to their invariant mass, the angular distributions of the four leptons and the dilepton pairs' invariant masses can be used to further discriminate signal from background and thus increase the signal sensitivity and reduce the statistical uncertainty in measurements, including the cross section, the mass, and the width of the resonance. Second, this extra information on angular correlations can be used to experimentally establish the consistency of the spin and parity quantum numbers with respect to the SM. This section describes how the full kinematic information from the production and decay can be encoded in a kinematic discriminant optimized for the separation of two processes, be it signal from background or between different signal hypotheses.

The kinematic properties of the SM Higgs boson or any non-SM exotic boson decay to the four-lepton final state has been extensively studied in Refs. [41-43,109-122]. Five angles $\vec{\Omega} \equiv\left(\theta^{*}, \Phi_{1}, \theta_{1}, \theta_{2}, \Phi\right)$ defined in Fig. 8 $[41,123]$ and the invariant masses of the lepton pairs, $m_{Z_{1}}$ and $m_{Z_{2}}$, fully describe the kinematic configuration of a four-lepton system in its center-of-mass frame, up to an 


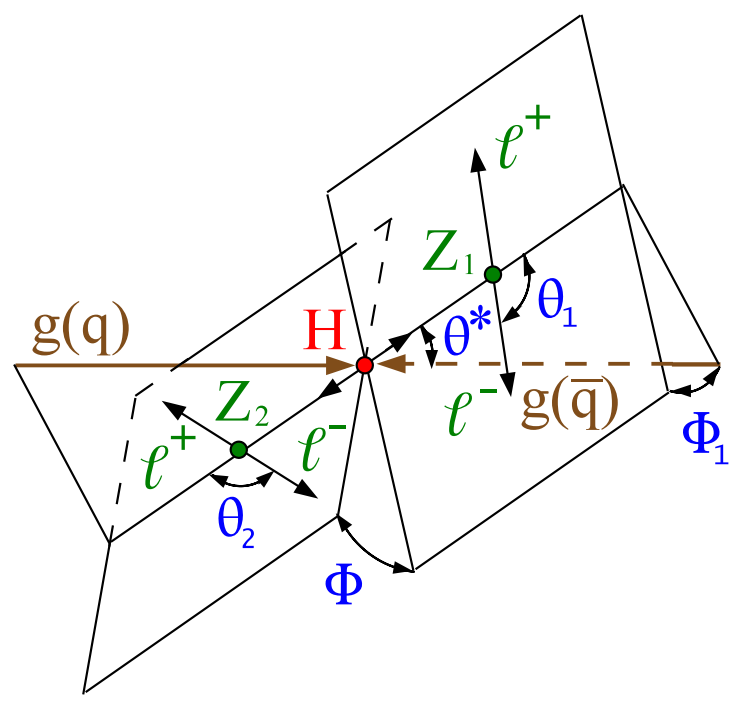

FIG. 8 (color online). Illustration of the production and decay of a particle $H, g g(q \bar{q}) \rightarrow H \rightarrow Z Z \rightarrow 4 \ell$, with the two production angles $\theta^{*}$ and $\Phi_{1}$ shown in the $H$ rest frame and three decay angles $\theta_{1}, \theta_{2}$, and $\Phi$ shown in the $Z_{1}, Z_{2}$, and $H$ rest frames, respectively.

arbitrary rotation around the beam axis. These observables provide significant discriminating power between signal and background, as well as between alternative signal models. A matrix-element likelihood approach is used to construct kinematic discriminants related to the decay observables $[20,31]$.

In addition to the four-lepton center-of-mass-frame observables, the four-lepton transverse momentum and rapidity are needed to completely define the system in the lab frame. The transverse momentum of the four-lepton system is used in the analysis as an independent observable because it is sensitive to the production mechanism of the Higgs boson, but it is not used in the spin-parity analysis. The four-lepton rapidity is not used because the discrimination power of this observable for events within the experimental acceptance is limited.

Kinematic discriminants are defined based on the event probabilities depending on the background $\left(\mathscr{P}_{\mathrm{bkg}}\right)$ or signal spin-parity $\left(J^{P}\right)$ hypotheses under consideration $\left(\mathscr{P}_{J^{P}}\right)$ :

$$
\begin{gathered}
\mathscr{P}_{\text {bkg }}=\mathscr{P}_{\mathrm{bkg}}\left(m_{Z_{1}}, m_{Z_{2}}, \vec{\Omega} \mid m_{4 \ell}\right) \times \mathscr{P}_{\mathrm{bkg}}^{\operatorname{mass}}\left(m_{4 \ell}\right), \\
\mathscr{P}_{J^{P}}=\mathscr{P}_{J^{P}}\left(m_{Z_{1}}, m_{Z_{2}}, \vec{\Omega} \mid m_{4 \ell}\right) \times \mathscr{P}_{\mathrm{sig}} \operatorname{mass}\left(m_{4 \ell} \mid m_{H}\right),
\end{gathered}
$$

where $\mathscr{P}^{\text {kin }}$ is the probability distribution of angular and mass observables $\left(\vec{\Omega}, m_{Z_{1}}, m_{Z_{2}}\right)$ computed from the LO matrix element squared for signal and $Z Z$ processes, and $\mathscr{P}^{\text {mass }}$ is the probability distribution of $m_{4 \ell}$ and is calculated using the parameterization described in Sec. XII A. Matrix elements for the signals are calculated with the assumption that $m_{H}=m_{4 \ell}$. The probability distributions for spin-0 resonances are independent of an assumed production mechanism. Only the dominant $q \bar{q} \rightarrow Z Z$ background is considered in the probability parameterization. For the reducible backgrounds, empirical templates derived from the data control samples defined in Sec. IX B are used to model the probability density functions of the kinematic discriminants, as described in Sec. XII.

For the alternative signal hypotheses, nine models have been tested, following the notations from Refs. [41,42]. The most general decay amplitude for a spin-0 boson decaying to two vector bosons can be defined as

$$
\begin{aligned}
A(H \rightarrow Z Z)= & v^{-1}\left(a_{1} m_{Z}^{2} \epsilon_{1}^{*} \epsilon_{2}^{*}+a_{2} f_{\mu \nu}^{*(1)} f^{*(2), \mu \nu}\right. \\
& \left.+a_{3} f_{\mu \nu}^{*(1)} \tilde{f}^{*(2), \mu \nu}\right),
\end{aligned}
$$

where $f^{(i), \mu \nu}=\epsilon_{i}^{\mu} q_{i}^{\nu}-\epsilon_{i}^{\nu} q_{i}^{\mu}$ is the field-strength tensor of a gauge boson with momentum $q_{i}$ and polarization vector $\epsilon_{i}$, $\tilde{f}_{\mu \nu}^{(i)}=1 / 2 \epsilon_{\mu \nu \alpha \beta} f^{(i), \alpha \beta}=\epsilon_{\mu \nu \alpha \beta} \epsilon_{i}^{\alpha} q_{i}^{\beta}$ is the conjugate field strength tensor, $f^{*}$ denotes the complex conjugate field strength tensor, and $v$ is the vacuum expectation value of the SM Higgs field. $\epsilon_{\mu \nu \alpha \beta}$ is the Levi-Civita completely antisymmetric tensor. The $a_{i}$ coefficients generally depend on $q_{i}^{2}$. In this analysis, we consider the lowest-dimension operators in the effective Lagrangian corresponding to each of the three unique Lorentz structures, therefore taking $a_{i}$ to be constant for the relevant range $q_{i}^{2}=m_{Z_{i}}^{2}<m_{H}^{2}$. The SM Higgs boson decay is dominated by the tree-level coupling $a_{1}$. The $0^{-}$model corresponds to a pseudoscalar (dominated by the $a_{3}$ coupling), while $0_{\mathrm{h}}^{+}$is a scalar (dominated by the $a_{2}$ coupling) not participating in the electroweak symmetry breaking, where $\mathrm{h}$ refers to higher-dimensional operators in Eq. (6) with respect to the SM Higgs boson. The spin- 0 signal models are simulated for the gluon fusion production process, and their kinematics in the boson center-of-mass frame is independent of the production mechanism.

The $1^{-}$and $1^{+}$hypotheses represent a vector and a pseudovector decaying to two $Z$ bosons. The spin-1 resonance models are simulated via the quark-antiquark production mechanism, as the gluon fusion production of such resonances is expected to be strongly suppressed. The spin-1 hypotheses are considered under the assumption that the resonance decaying into $4 \ell$ is not necessarily the same resonance observed in the $H \rightarrow \gamma \gamma$ channel $[19,20]$, as $J=1$ in the latter case is prohibited by the Landau-Yang theorem [124,125]. This also provides a test of the spin-1 hypothesis in an independent way.

The spin- 2 model with minimal couplings, $2_{\mathrm{m}}^{+}$, represents a massive graviton-like boson $X$ suggested, for example, in models with warped extra dimensions (ED) $[126,127]$, where gluon fusion is the dominant process. For completeness, $100 \%$ quark-antiquark annihilation is also considered, which provides a projection of the spin of the resonance on the parton collision axis equal to 1 , instead of 2 , as in the case of gluon fusion with minimal couplings. 
A modified minimal coupling model $2_{\mathrm{b}}^{+}$is also considered, where the SM fields are allowed to propagate in the bulk of the ED [128], corresponding to $g_{1} \ll g_{5}$ in the $X Z Z$ coupling for the $2_{\mathrm{m}}^{+}$model, where the $g_{i}$ 's are the couplings in the effective Lagrangian of Ref. [42]. Finally, two spin-2 models with higher-dimension operators are considered with both positive and negative parity, $2_{\mathrm{h}}^{+}$and $2_{\mathrm{h}}^{-}$, corresponding to the $g_{4}$ and $g_{8}$ couplings. The $2_{\mathrm{b}}^{+}, 2_{\mathrm{h}}^{+}$, and $2_{\mathrm{h}}^{-}$ resonances are assumed to be produced in gluon fusion. The above list of the spin- 2 models does not exhaust all possible scenarios, nor does it cover possible mixed states. However, it does provide a representative sample of spin-2 alternatives to the $J^{P}=0^{+}$hypothesis.

For discrimination between the SM Higgs boson $\left(J^{P}=0^{+}\right)$and the SM backgrounds (nonresonant $\mathrm{ZZ}$ and reducible backgrounds), an observable is created from the probability distributions in Eqs. (4) and (5):

$$
\mathscr{D}_{\mathrm{bkg}}^{\mathrm{kin}}=\frac{\mathscr{P}_{0^{+}}^{\mathrm{kin}}}{\mathscr{P}_{0^{+}}^{\mathrm{k}}+\mathscr{P}_{\mathrm{bkg}} \mathrm{kin}}=\left[1+\frac{\mathscr{P}_{\mathrm{bkg}}^{\mathrm{kin}}\left(m_{Z_{1}}, m_{Z_{2}}, \vec{\Omega} \mid m_{4 \ell}\right)}{\mathscr{P}_{0^{+}}\left(m_{Z_{1}}, m_{Z_{2}}, \vec{\Omega} \mid m_{4 \ell}\right)}\right]^{-1} .
$$

The discriminant defined this way does not carry direct discrimination power based on the four-lepton mass $m_{4 \ell}$ between the signal and the background. Hence, it can be used as a second discriminating observable in addition to the $m_{4 \ell}$ distribution. The $\mathscr{P}_{i}$ 's are normalized with additional constant factors for a given value of $m_{4 \ell}$, such that the ratio of probabilities is scaled by a constant factor leading to probabilities $P(\mathscr{D}>0.5 \mid H)=P(\mathscr{D}<0.5 \mid \mathrm{bkg})$.

In this analysis, the SM Higgs boson signal is distinguished simultaneously from the background and from alternative signal hypotheses. The former is separated with $\mathscr{D}_{\text {bkg }}$, and the latter with $\mathscr{D}_{J^{p}}$ observables constructed from the background, signal, and the probability of the alternative hypotheses defined in Eqs. (4) and (5). The $\mathscr{D}_{\mathrm{bkg}}$ observable extends $\mathscr{D}_{\mathrm{bkg}}^{\mathrm{kin}}$ defined in Eq. (7) with the fourlepton mass probability for separation at a fixed value of the mass $m_{0^{+}}$:

$\mathscr{D}_{\mathrm{bkg}}=\left[1+\frac{\mathscr{P}_{\mathrm{bkg}}^{\mathrm{kin}}\left(m_{Z_{1}}, m_{Z_{2}}, \vec{\Omega} \mid m_{4 \ell}\right) \times \mathscr{P}_{\mathrm{bkg}}^{\operatorname{mass}}\left(m_{4 \ell}\right)}{\mathscr{P}_{0^{+}}^{\mathrm{kin}}\left(m_{Z_{1}}, m_{Z_{2}}, \vec{\Omega} \mid m_{4 \ell}\right) \times \mathscr{P}_{\text {sig }}^{\operatorname{mass}}\left(m_{4 \ell} \mid m_{0^{+}}\right)}\right]^{-1}$.

The other observable discriminates between the SM Higgs boson and the alternative signal hypothesis:

$$
\mathscr{D}_{J^{P}}=\left[1+\frac{\mathscr{P}_{J^{P}}^{\mathrm{kin}}\left(m_{Z_{1}}, m_{Z_{2}}, \vec{\Omega} \mid m_{4 \ell}\right)}{\mathscr{P}_{0^{+}}\left(m_{Z_{1}}, m_{Z_{2}}, \vec{\Omega} \mid m_{4 \ell}\right)}\right]^{-1}
$$

The spin-0 discriminants $\mathscr{D}_{0^{-}}$and $\mathscr{D}_{0^{+}}$are independent of any production mechanism, since in the production of a spin- 0 particle the angular decay variables are independent of production mechanism. This is not the case for the spin-1 and spin-2 signal hypotheses. Therefore, it is desirable to test the spin- 1 and spin-2 hypotheses in a way that does not depend on assumptions about the production mechanism. This is achieved by either averaging over the spin degrees of freedom of the produced boson or, equivalently, integrating the matrix elements squared over the production angles $\cos \theta^{*}$ and $\Phi_{1}$ [48]. With the latter, the discriminants are defined as

$$
\mathscr{D}_{\mathrm{bkg}}^{\mathrm{dec}}=\left[1+\frac{\frac{1}{4 \pi} \int d \Phi_{1} d \cos \theta^{*} \mathscr{P}_{\mathrm{bkg}}^{\mathrm{kin}}\left(m_{Z_{1}}, m_{Z_{2}}, \vec{\Omega} \mid m_{4 \ell}\right) \times \mathscr{P}_{\mathrm{bkg}}^{\operatorname{mass}}\left(m_{4 \ell}\right)}{\mathscr{P}_{0^{+}}\left(m_{Z_{1}}, m_{Z_{2}}, \vec{\Omega} \mid m_{4 \ell}\right) \times \mathscr{P}_{\mathrm{sig}}\left(m_{4 \ell} \mid m_{0^{+}}\right)}\right]^{-1},
$$

$$
\mathscr{D}_{J^{P}}^{\mathrm{dec}}=\left[1+\frac{\frac{1}{4 \pi} \int d \Phi_{1} d \cos \theta^{*} \underset{J^{P}}{\operatorname{kin}}\left(m_{Z_{1}}, m_{Z_{2}}, \vec{\Omega} \mid m_{4 \ell}\right)}{\mathscr{P}_{0^{+}}\left(m_{Z_{1}}, m_{Z_{2}}, \vec{\Omega} \mid m_{4 \ell}\right)}\right]^{-1} .
$$

The superscript "dec" indicates that these discriminants use decay-only information. The probabilities for spin- 0 resonances are already independent of the production mechanism; however, their distributions, for all the $J^{P}$ hypotheses, do carry some production dependence due to detector and analysis acceptance effects. Such productiondependent variations in the discriminant distribution shapes are found to be small and are treated as systematic uncertainties.

Table II summarizes all kinematic observables used in this analysis, for different purposes. To make an optimal use of the available information, the distribution of these observables is used without any selection in a fit.

This analysis uses the matrix-element likelihood approach (MELA) framework [20,42,43], with the matrix elements for different signal models taken from JHUGEN [41-43] and the matrix element for the $q \bar{q} \rightarrow Z Z$ background taken from MCFM [104-106]. Within the MELA framework, an analytical parameterization of matrix elements for signal $[41,42]$ and background [120] was adopted in the previous analyses of CMS data with results reported in Refs. [20,31]. The above matrix-element calculations are validated against each other and also tested with the matrixelement kinematic discriminant (MEKD) framework [121], based on MADGRAPH [70] and FEYNRULES [129], and with a stand-alone framework implementation of MADGRAPH. The inclusion of the lepton interference in the kinematic 
TABLE II. List of observables and kinematic discriminants used for signal versus background separation and studies of the properties of the observed resonance. The alternative hypotheses for $J=0$ are independent of the production mechanism without the need of integrating out the production angles $\cos \theta^{*}$ and $\Phi_{1}$.

\begin{tabular}{|c|c|}
\hline Discriminant & Note \\
\hline \multicolumn{2}{|c|}{ Observables used for the signal-strength measurement } \\
\hline$m_{4 \ell}$ & Four-lepton invariant mass, main background discrimination. \\
\hline $\mathscr{D}_{\mathrm{bkg}}^{\mathrm{kin}}$ & Discriminate SM Higgs boson against $Z Z$ background. \\
\hline $\mathscr{D}_{\text {jet }}^{\text {ong }}$ & Linear discriminant, uses jet information to identify VBF topology. \\
\hline$p_{\mathrm{T}}^{4 \ell}$ & $p_{\mathrm{T}}$ of the $4 \ell$ system, discriminates between production mechanisms. \\
\hline \multicolumn{2}{|c|}{ Observables used in the spin-parity hypothesis testing } \\
\hline $\begin{array}{l}\mathscr{D}_{\text {bkg }} \\
\mathscr{D}_{1^{-}}\end{array}$ & $\begin{array}{l}\text { Discriminates SM Higgs boson against } Z Z \text { background, includes } m_{4 \ell} \text {. } \\
\text { Exotic vector }\left(1^{-}\right), q \bar{q} \text { annihilation. }\end{array}$ \\
\hline $\mathscr{D}_{1^{+}}$ & Exotic pseudovector $\left(1^{+}\right), q \bar{q}$ annihilation. \\
\hline $\mathscr{D}_{2_{m}^{+}}^{g g}$ & Graviton-like with minimal couplings $\left(2_{\mathrm{m}}^{+}\right)$, gluon fusion. \\
\hline $\mathscr{D}_{2_{\mathrm{m}}^{+}}^{q \bar{q}}$ & Graviton-like with minimal couplings $\left(2_{\mathrm{m}}^{+}\right), q \bar{q}$ annihilation. \\
\hline $\mathscr{D}_{2^{+}}^{g g}$ & Graviton-like with $\mathrm{SM}$ in the bulk $\left(2_{\mathrm{b}}^{+}\right)$, gluon fusion. \\
\hline $\mathscr{D}_{2^{+}}^{g g}$ & Tensor with higher-dimension operators $\left(2_{\mathrm{h}}^{+}\right)$, gluon fusion. \\
\hline $\mathscr{D}_{2_{\mathrm{h}}^{-}}^{g \mathrm{~g}}$ & Pseudotensor with higher-dimension operators $\left(2_{\mathrm{h}}^{-}\right)$, gluon fusion. \\
\hline \multicolumn{2}{|c|}{ Production-independent observables used in the spin-parity hypothesis testing } \\
\hline $\mathscr{D}_{0^{-}}$ & Pseudoscalar $\left(0^{-}\right)$, discriminates against SM Higgs boson. \\
\hline $\mathscr{D}_{0_{\mathrm{h}}^{+}}$ & Non-SM scalar with higher-dimension operators $\left(0_{\mathrm{h}}^{+}\right)$ \\
\hline $\begin{array}{l}\mathscr{D}_{\mathrm{bkg}}^{\mathrm{dec}} \\
\mathscr{D}_{1-c}^{\text {dec }}\end{array}$ & $\begin{array}{l}\text { Discriminates against } Z Z \text { background, includes } m_{4 \ell} \text {, excludes } \cos \theta^{*}, \Phi_{1} \text {. } \\
\text { Exotic vector }\left(1^{-}\right) \text {, decay-only information. }\end{array}$ \\
\hline $\begin{array}{l}1_{1-}^{-} \\
\mathscr{D}_{1^{+}}^{\text {dec }}\end{array}$ & Exotic pseudovector $\left(1^{+}\right)$, decay-only information. \\
\hline $\mathscr{D}_{2_{\mathrm{m}}^{+}}^{\mathrm{dec}}$ & Graviton-like with minimal couplings $\left(2_{\mathrm{m}}^{+}\right)$, decay-only information. \\
\hline
\end{tabular}

discriminant parameterization is a small improvement in the expected separation significance of $\sim 3 \%$ for spin-0 models with respect to earlier published results [20,31], as indicated by cross-checks with generator-based matrixelement calculations performed in the MELA and MEKD frameworks within studies reported in Ref. [31].

Detector acceptance effects approximately cancel in the probability ratios, such as those in Eq. (7). In principle, the kinematic discriminants could be modified to account for detector resolution effects. However, the matrix-element approach with detector transfer functions modeling detector resolution effects showed nearly identical performance. This is not unexpected for leptons, as their resolutions are of $\mathcal{O}(1 \%)$ and are therefore negligible.

In order to provide additional validation of the kinematic discriminants, machine-learning techniques have been used to construct discriminants. Two techniques have been used: the Bayesian neural networks (BNN) framework [130,131] and the BDT framework $[88,132,133]$. In the BNN framework, a Bayesian procedure is used to create a posterior probability density over the space of neural network parameters. This probability density is then used to calculate a BNN. In both frameworks, a discriminant is built using the four-lepton angular and mass variables, and the output is used in the same way as the $\mathscr{D}_{\mathrm{bkg}}^{\mathrm{kin}}$ in the analysis described above. The BNN and BDT discriminants are trained using simulated samples to discriminate signatures for signal events from those for background events or to discriminate between different signal hypotheses. The MC samples generated for training are based on the same matrix elements for signal and background as used in the analysis and include the effects of the full detector simulation. The machinetrained discriminants are found to give similar performance to the matrix-element approaches described above.

\section{YIELDS AND KINEMATIC DISTRIBUTIONS}

The signal and background yields are extracted from a fit to the invariant mass and other kinematic properties, characterizing the decay of the Higgs boson candidate and its production mechanism. The expected distributions of signal and background components are used as probability density functions in the likelihood function. Simulation and control samples from data are used to estimate the initial fit values for the signal and background yields.

The background from $Z Z$ and $Z+X$ processes dominates after the event selection. The reconstructed fourlepton invariant mass distribution for the combined $4 e$, $2 e 2 \mu$, and $4 \mu$ channels is shown in Fig. 9 and compared with the expectations from background processes. Here, and in the other figures of this section, the normalization and shape of the $Z Z$ background and the signal 


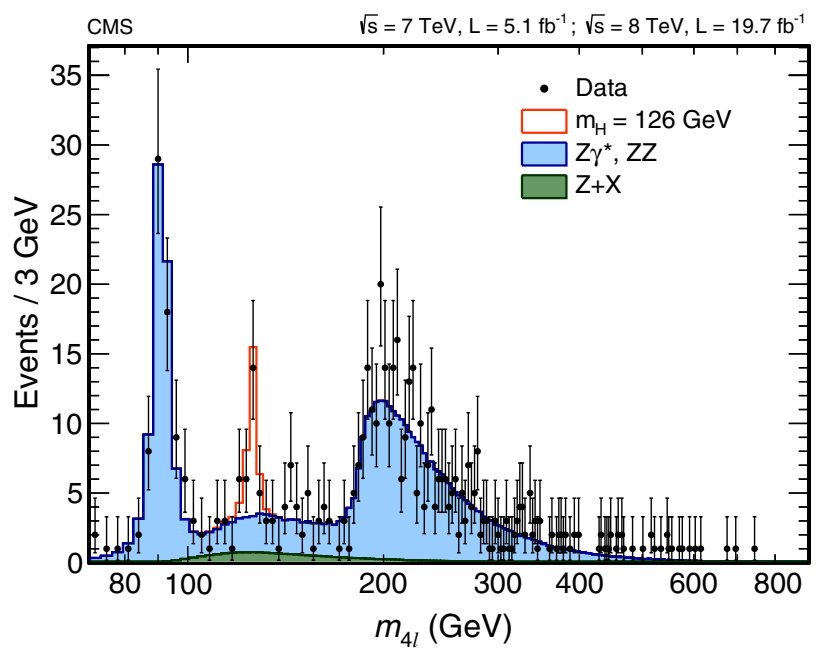

FIG. 9 (color online). Distribution of the four-lepton reconstructed mass in the full mass range $70<m_{4 \ell}<1000 \mathrm{GeV}$ for the sum of the $4 e, 2 e 2 \mu$, and $4 \mu$ channels. Points with error bars represent the data, shaded histograms represent the backgrounds, and the unshaded histogram represents the signal expectation for a mass hypothesis of $m_{H}=126 \mathrm{GeV}$. Signal and the $Z Z$ background are normalized to the SM expectation; the $Z+X$ background to the estimation from data. The expected distributions are presented as stacked histograms. No events are observed with $m_{4 \ell}>800 \mathrm{GeV}$.

$\left(m_{H}=126 \mathrm{GeV}\right)$ are obtained from simulation, while the normalization and shape of the reducible background is estimated from control samples in data, as described in Sec. IX B. The error bars on data points are asymmetric Poisson uncertainties that cover the $68 \%$ probability interval around the central value [134]. A clear peak around $m_{4 \ell}=126 \mathrm{GeV}$ is seen, not expected from background processes, confirming with a larger data sample the results reported in Refs. [19-21,31]. The observed distribution is in good agreement with the expected backgrounds and a narrow resonance compatible with the SM Higgs boson with $m_{H}$ around $126 \mathrm{GeV}$. The $Z \rightarrow 4 \ell$ resonance peak at $m_{4 \ell}=m_{Z}$ is observed in agreement with simulation. The measured distribution at masses greater than $2 m_{Z}$ is dominated by the irreducible $Z Z$ background, where the two $Z$ bosons are produced on shell.

The number of candidates observed in data as well as the expected yields for background and several SM Higgs boson mass hypotheses are reported in Table III, for $m_{4 \ell}>100 \mathrm{GeV}$. The observed event rates for the various channels are compatible with SM background expectations in the $m_{4 \ell}$ region above $2 m_{Z}$, while a deviation is observed in the lower region. Given that the excess of events observed in the $4 \ell$ mass spectrum is localized in a narrow region in the vicinity of $126 \mathrm{GeV}$, the events expected in a narrower range, $121.5<m_{4 \ell}<130.5 \mathrm{GeV}$, are reported in Table IV. Table V reports the breakdown of the events observed in data and the expected background yields in the same $m_{4 \ell}$ region in the two analysis categories, together
TABLE III. The number of observed candidate events compared to the mean expected background and signal rates for each final state. Uncertainties include statistical and systematic sources. The results are given integrated over the full mass measurement range $m_{4 \ell}>100 \mathrm{GeV}$ and for 7 and $8 \mathrm{TeV}$ data combined.

\begin{tabular}{lcccc}
\hline \hline Channel & $4 e$ & $2 e 2 \mu$ & $4 \mu$ & $4 \ell$ \\
\hline$Z Z$ background & $77 \pm 10$ & $191 \pm 25$ & $119 \pm 15$ & $387 \pm 31$ \\
$Z+X$ background & $7.4 \pm 1.5$ & $11.5 \pm 2.9$ & $3.6 \pm 1.5$ & $22.6 \pm 3.6$ \\
All backgrounds & $85 \pm 11$ & $202 \pm 25$ & $123 \pm 15$ & $410 \pm 31$ \\
$m_{H}=500 \mathrm{GeV}$ & $5.2 \pm 0.6$ & $12.2 \pm 1.4$ & $7.1 \pm 0.8$ & $24.5 \pm 1.7$ \\
$m_{H}=800 \mathrm{GeV}$ & $0.7 \pm 0.1$ & $1.6 \pm 0.2$ & $0.9 \pm 0.1$ & $3.1 \pm 0.2$ \\
Observed & 89 & 247 & 134 & 470 \\
\hline \hline
\end{tabular}

TABLE IV. The number of observed candidate events compared to the mean expected background and signal rates for each final state. Uncertainties include statistical and systematic sources. The results are integrated over the mass range from 121.5 to $130.5 \mathrm{GeV}$ and for 7 and $8 \mathrm{TeV}$ data combined.

\begin{tabular}{lcccc}
\hline \hline Channel & $4 e$ & $2 e 2 \mu$ & $4 \mu$ & $4 \ell$ \\
\hline$Z Z$ background & $1.1 \pm 0.1$ & $3.2 \pm 0.2$ & $2.5 \pm 0.2$ & $6.8 \pm 0.3$ \\
$Z+X$ background & $0.8 \pm 0.2$ & $1.3 \pm 0.3$ & $0.4 \pm 0.2$ & $2.6 \pm 0.4$ \\
All backgrounds & $1.9 \pm 0.2$ & $4.6 \pm 0.4$ & $2.9 \pm 0.2$ & $9.4 \pm 0.5$ \\
$m_{H}=125 \mathrm{GeV}$ & $3.0 \pm 0.4$ & $7.9 \pm 1.0$ & $6.4 \pm 0.7$ & $17.3 \pm 1.3$ \\
$m_{H}=126 \mathrm{GeV}$ & $3.4 \pm 0.5$ & $9.0 \pm 1.1$ & $7.2 \pm 0.8$ & $19.6 \pm 1.5$ \\
Observed & 4 & 13 & 8 & 25 \\
\hline \hline
\end{tabular}

with the expected yield for a SM Higgs boson with $m_{H}=126 \mathrm{GeV}$, split by production mechanism. The $m_{4 \ell}$ distribution for the sum of the $4 e, 2 e 2 \mu$, and $4 \mu$ channels, in the mass region $70<m_{4 \ell}<180 \mathrm{GeV}$, is shown in Fig. 10. Figure 11 shows the reconstructed

TABLE V. The number of observed candidate events compared to the mean expected background and signal rates for the sum of the three final states for each of the two analysis categories. Uncertainties include statistical and systematic sources. The results are integrated over the mass range from 121.5 to $130.5 \mathrm{GeV}$ and for 7 and $8 \mathrm{TeV}$ data combined. The expected signal yield for a SM Higgs boson with $m_{H}=126 \mathrm{GeV}$ is reported, broken down by the production mechanism.

\begin{tabular}{lcc}
\hline \hline Category & 0/1-jet & Dijet \\
\hline$Z Z$ background & $6.4 \pm 0.3$ & $0.38 \pm 0.02$ \\
$Z+X$ background & $2.0 \pm 0.3$ & $0.5 \pm 0.1$ \\
All backgrounds & $8.5 \pm 0.5$ & $0.9 \pm 0.1$ \\
$g g H$ & $15.4 \pm 1.2$ & $1.6 \pm 0.3$ \\
$t \bar{t} H$ & $\cdots$ & $0.08 \pm 0.01$ \\
$\mathrm{VBF}$ & $0.70 \pm 0.03$ & $0.87 \pm 0.07$ \\
$W H$ & $0.28 \pm 0.01$ & $0.21 \pm 0.01$ \\
$Z H$ & $0.21 \pm 0.01$ & $0.16 \pm 0.01$ \\
All signal, $m_{H}=126 \mathrm{GeV}$ & $16.6 \pm 1.3$ & $3.0 \pm 0.4$ \\
Observed & 20 & 5 \\
\hline \hline
\end{tabular}




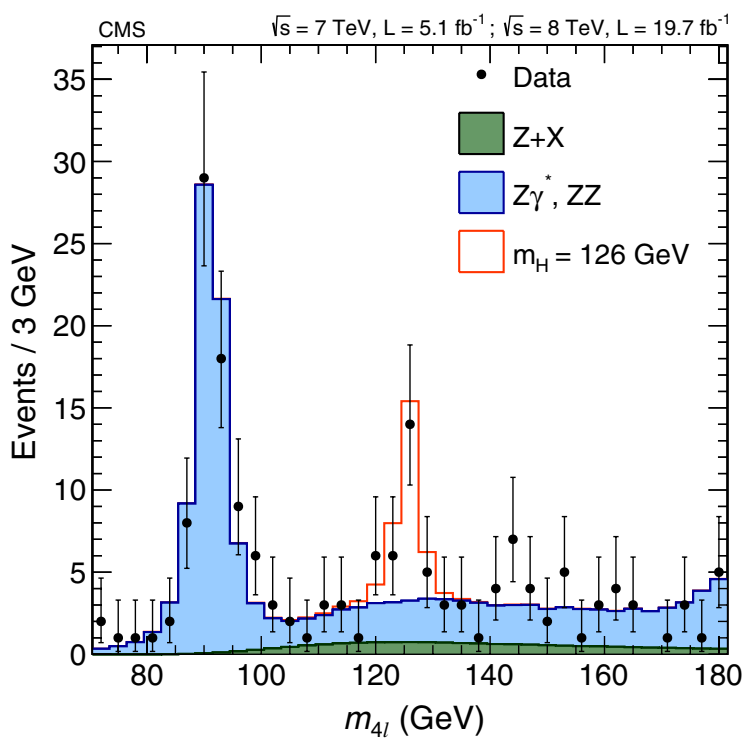

FIG. 10 (color online). Distribution of the four-lepton reconstructed mass for the sum of the $4 e, 2 e 2 \mu$, and $4 \mu$ channels for the mass region $70<m_{4 \ell}<180 \mathrm{GeV}$. Points with error bars represent the data, shaded histograms represent the backgrounds, and the unshaded histogram represents the signal expectation for a mass hypothesis of $m_{H}=126 \mathrm{GeV}$. Signal and the $Z Z$ background are normalized to the SM expectation; the $Z+X$ background to the estimation from data.

invariant masses of the $Z_{1}$ and $Z_{2}$ in a $m_{4 \ell}$ range between 121.5 and $130.5 \mathrm{GeV}$.

The distributions of the $\mathscr{D}_{\mathrm{bkg}}^{\mathrm{kin}}$ versus $m_{4 \ell}$ are shown for the selected events and compared to the SM background expectation in Fig. 12. The distribution of events in the $\left(m_{4 \ell}, \mathscr{D}_{\mathrm{bkg}}^{\mathrm{kin}}\right)$ plane agrees well with the SM background expectation in the high-mass range [Fig. 12 (bottom)], while discrepancies in the two-dimensional plane are observed in the low-mass range $110<m_{4 \ell}<180 \mathrm{GeV}$ [Fig. 12 (top)], indicative of the presence of a signal. Figure 13 (top) shows the same data points as in Fig. 12 (top), but compared with the expected distribution from SM backgrounds plus the contribution of a Higgs boson with $m_{H}=126 \mathrm{GeV}$. A signal-like clustering of events is apparent at high values of $\mathscr{D}_{\mathrm{bkg}}^{\mathrm{kin}}$ and for $m_{4 \ell} \approx 126 \mathrm{GeV}$. Figure 13 (bottom) shows the distribution of the kinematic discriminant $\mathscr{D}_{\mathrm{bkg}}^{\mathrm{kin}}$ in the mass region $121.5<m_{4 \ell}<$ $130.5 \mathrm{GeV}$.

The distribution of the transverse momentum of the $4 \ell$ system in the 0/1-jet category and its joint distribution with $m_{4 \ell}$ are shown in Fig. 14. The $p_{\mathrm{T}}$ spectrum shows good agreement with a SM Higgs boson hypothesis with $m_{H}=126 \mathrm{GeV}$ in the $0 / 1$-jet category with few events having $p_{\mathrm{T}}>60 \mathrm{GeV}$, where $\mathrm{VBF}$ and $\mathrm{VH}$ production are relatively more relevant. In order to compare the $p_{\mathrm{T}}$ spectrum in data with the SM Higgs boson distribution more quantitatively, a background subtraction using the ${ }_{s} \mathscr{P}$ lot weighting technique [135] is performed. The event weights, related to the probability for each event to be signal-like or background-like, are computed according to the one-dimensional likelihood based on the $m_{4 \ell}$ distribution, which shows a small correlation with the four-lepton $p_{\mathrm{T}}^{4 \ell}$. The weighted distribution has the property that it corresponds to the signal-only distribution and is normalized to the fitted signal yield. The background-subtracted weighted $p_{\mathrm{T}}^{4 \ell}$ distribution is shown in Fig. 15.

The distribution of the production mechanism discriminant in the dijet category and its joint distribution with $m_{4 \ell}$ are shown in Fig. 16. Good agreement is found with the expectation from simulation, which predicts a negligible background and a fraction of $42 \%$ of the signal events
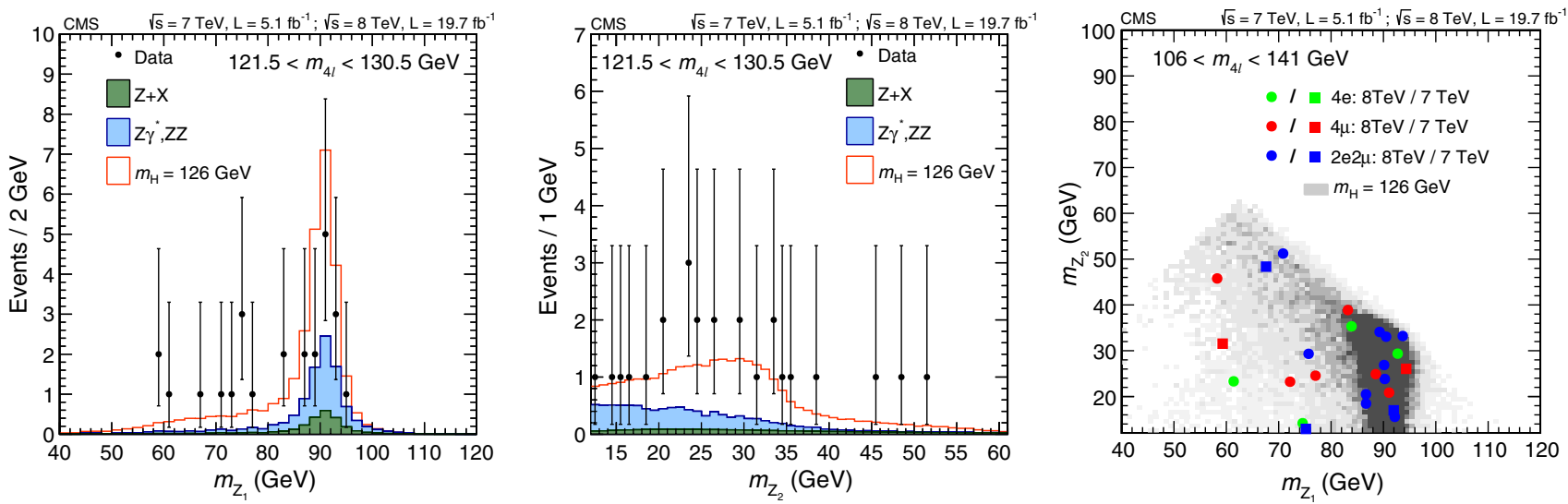

FIG. 11 (color online). Distribution of (left) the $Z_{1}$ and (center) the $Z_{2}$ reconstructed invariant masses, in the mass region $121.5<m_{4 \ell}<130.5 \mathrm{GeV}$, for the sum of the four-lepton channels. Points represent the data, and shaded histograms represent the background. The signal expectation at $m_{H}=126 \mathrm{GeV}$ is shown as the unshaded histogram. Signal and background histograms are stacked. (right) Two-dimensional distribution of the two variables in the mass region $106<m_{4 \ell}<141 \mathrm{GeV}$, corresponding to the range used in the signal extraction for $m_{H}=126 \mathrm{GeV}$, for the sum of the $4 \ell$ channels. The signal expectation at $m_{H}=126 \mathrm{GeV}$ is shown as the grey scale. 

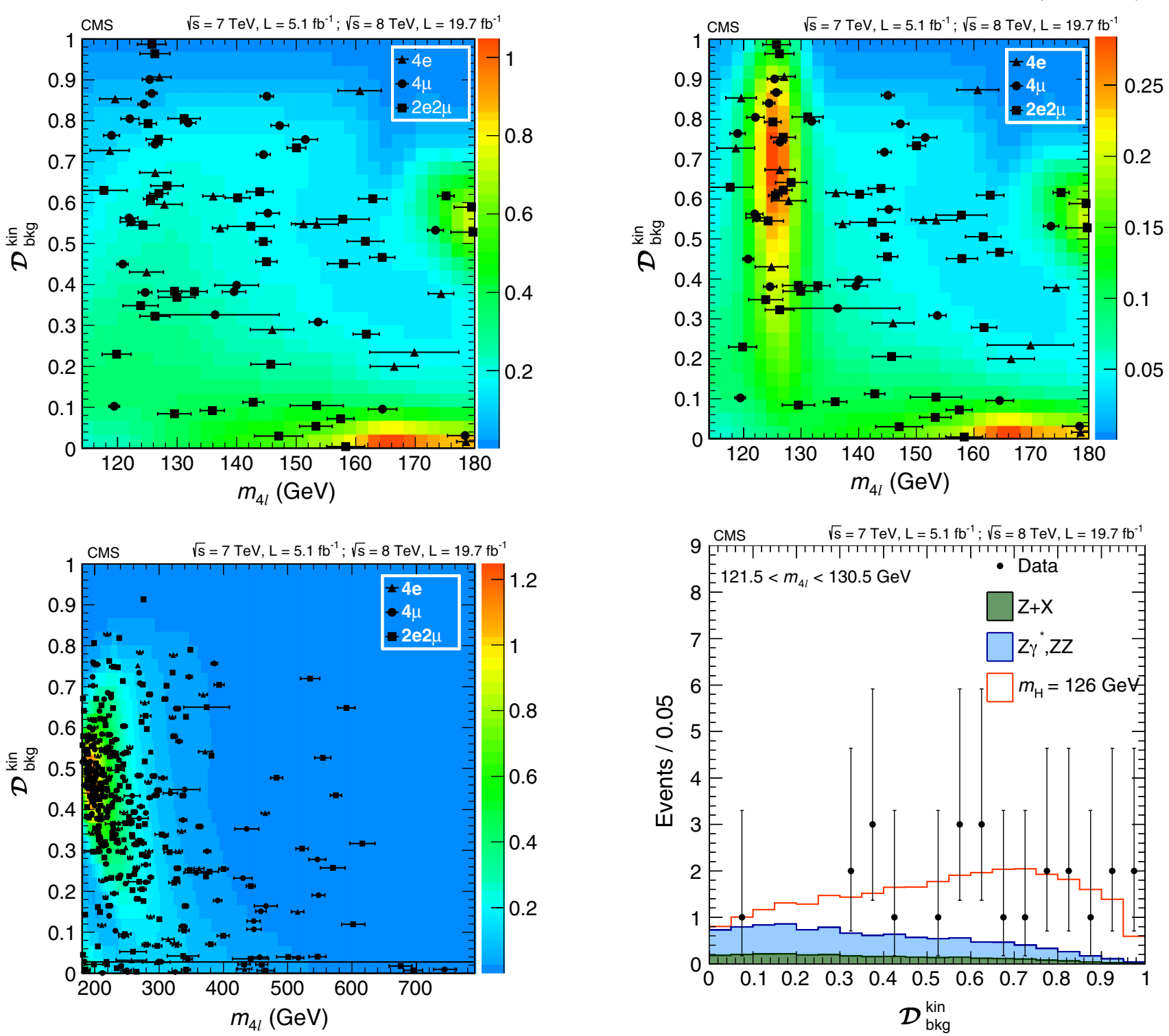

FIG. 12 (color online). Distribution of the kinematic discriminant $\mathscr{D}_{\mathrm{bkg}}^{\mathrm{kin}}$ versus the four-lepton reconstructed mass $m_{4 \ell}$ in the (top) lowmass and (bottom) high-mass regions. The color scale represents the expected relative density in linear scale (in arbitrary units) of background events. The points show the data and the measured per-event invariant mass uncertainties as horizontal bars. One $2 e 2 \mu$ event with $m_{4 \ell} \approx 220 \mathrm{GeV}$ and small $\mathscr{D}_{\mathrm{bkg}}^{\mathrm{kin}}$ has a huge mass uncertainty, and it is displayed as the horizontal line. No events are observed for $m_{4 \ell}>800 \mathrm{GeV}$.

arising from vector-boson-induced production (VBF and $\mathrm{VH})$. No events with a high rank of the $\mathscr{D}_{\text {jet }}\left(\mathscr{D}_{\text {jet }}>0.5\right)$ discriminant are observed.

\section{HIGGS BOSON PROPERTIES MEASUREMENT}

In this section, the fit models used to perform the measurements in the $H \rightarrow Z Z \rightarrow 4 \ell$ channel, based on the observables defined in the previous sections, are

FIG. 13 (color online). (top) Distribution of $\mathscr{D}_{\mathrm{bkg}}^{\mathrm{kin}}$ versus $m_{4 \ell}$ in the low-mass range with colors shown for the expected relative density in linear scale (in arbitrary units) of background plus the Higgs boson signal for $m_{H}=126 \mathrm{GeV}$. The points show the data, and horizontal bars represent the measured mass uncertainties. (bottom) Distribution of the kinematic discriminant $\mathscr{D}_{\mathrm{bkg}}^{\mathrm{kin}}$ for events in the mass region $121.5<m_{4 \ell}<130.5 \mathrm{GeV}$. Points with error bars represent the data, shaded histograms represent the backgrounds, and the unshaded histogram the signal expectation. Signal and background histograms are stacked.

presented. Then, the systematic uncertainties effects considered in the fits for both assessing the presence of a signal and performing the measurement of different properties are described.

\section{A. Multidimensional likelihoods}

The properties of interest to be measured in this analysis, such as the signal and background yields, the mass and 

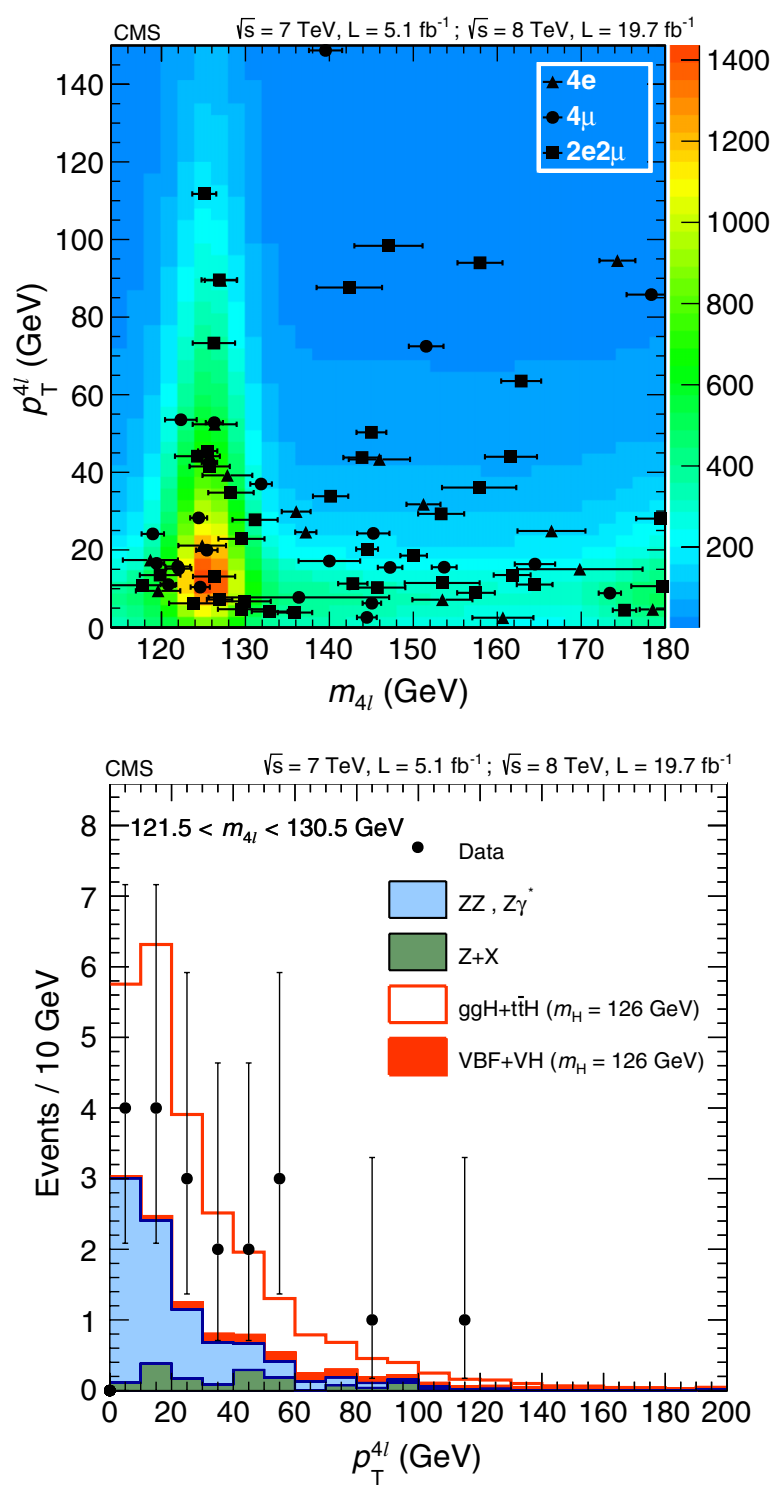

FIG. 14 (color online). (top) Distribution of $p_{\mathrm{T}}^{4 \ell}$ versus $m_{4 \ell}$ in the low-mass-range $0 / 1$-jet category with colors shown for the expected relative density in linear scale (in arbitrary units) of background plus the Higgs boson signal for $m_{H}=126 \mathrm{GeV}$. No events are observed for $p_{\mathrm{T}}>150 \mathrm{GeV}$. The points show the data, and horizontal bars represent the measured mass uncertainties. (bottom) Distribution of $p_{\mathrm{T}}^{4 \ell}$ in the $0 / 1$-jet category for events in the mass region $121.5<m_{4 \ell}<130.5 \mathrm{GeV}$. Points with error bars represent the data, shaded histograms represent the backgrounds, and the red histograms represent the signal expectation, broken down by production mechanism. Signal and background histograms are stacked.

width of the resonance, and the spin-parity quantum numbers, are determined with unbinned maximum-likelihood fits performed to the selected events. The fits include probability density functions for five signal components (gluon fusion, $\mathrm{VBF}, W H, Z H$, and $t \bar{t} H$ productions) and three background processes $(q \bar{q} \rightarrow Z Z, g g \rightarrow Z Z$, and $Z+X)$. The normalizations of these components

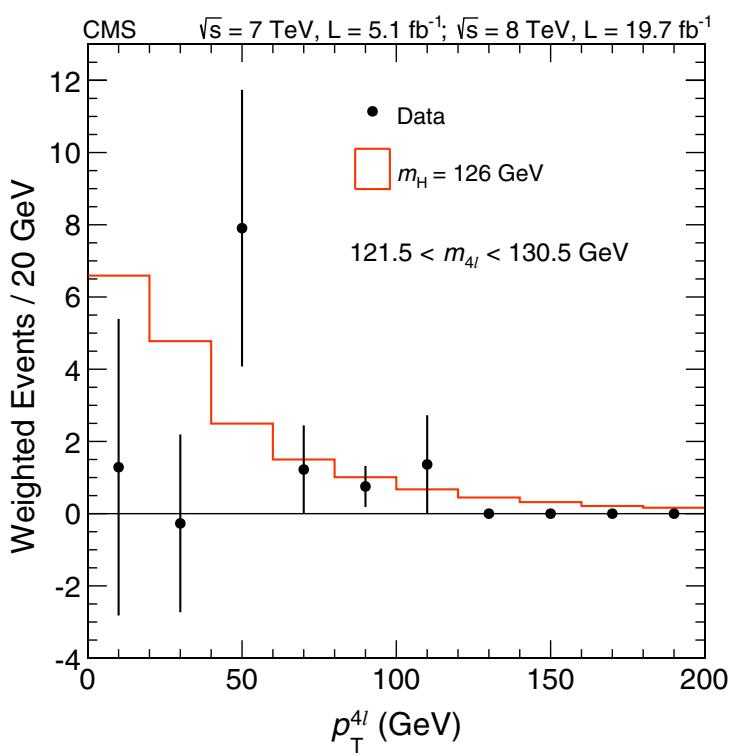

FIG. 15 (color online). ${ }_{s} \mathscr{P} l o t$ signal-weighted distribution of the four-lepton system $p_{\mathrm{T}}^{4 \ell}$ for all the selected events in the mass region $121.5<m_{4 \ell}<130.5 \mathrm{GeV}$. The red solid line represents the expectation from a SM Higgs boson.

and systematic uncertainties are introduced in the fits as nuisance parameters, assuming log-normal a priori probability distributions, and are profiled during the minimization. The shapes of the probability density functions for the event observables are also varied within alternative ones, according to the effect induced by experimental or theoretical systematic uncertainties $[30,136]$. Depending on the specific result to be extracted, different multidimensional models, using different sets of discriminating variables, are used. The dimension refers to the number of input variables used in the likelihood function. In the cases where one of the discriminants listed in Table II is used, this observable typically combines more than one discriminating variable. Each of these models is outlined below:

(1) For the assessment of exclusion limits as a function of $m_{H}$, the signal significance, and the measurement of the signal strength, $\mu \equiv \sigma / \sigma_{\mathrm{SM}}$, defined as the measured cross section times the branching fraction into $Z Z$, relative to the expectation for the SM Higgs boson, the following 3D likelihood functions are used:

$$
\begin{aligned}
\mathscr{L}_{3 D}^{\mu} \equiv & \mathscr{L}_{3 D}^{\mu, 0 / 1 \text {-jet }}\left(m_{4 \ell}, \mathscr{D}_{\mathrm{bkg}}^{\mathrm{kin}}, p_{\mathrm{T}}^{4 \ell}\right) \\
= & \mathscr{P}\left(m_{4 \ell} \mid m_{H}, \Gamma\right) \mathscr{P}\left(\mathscr{D}_{\mathrm{bkg}}^{\mathrm{kin}} \mid m_{4 \ell}\right) \\
& \times \mathscr{P}\left(p_{\mathrm{T}}^{4 \ell} \mid m_{4 \ell}\right), \\
\mathscr{L}_{3 D}^{\mu} \equiv & \mathscr{L}_{3 D}^{\mu \text {,ijet }}\left(m_{4 \ell}, \mathscr{D}_{\mathrm{bkg}}^{\mathrm{kin}}, \mathscr{D}_{\mathrm{jet}}\right) \\
= & \mathscr{P}\left(m_{4 \ell} \mid m_{H}, \Gamma\right) \mathscr{P}\left(\mathscr{D}_{\mathrm{bkg}}^{\mathrm{kin}} \mid m_{4 \ell}\right) \\
& \times \mathscr{P}\left(\mathscr{D}_{\mathrm{jet}} \mid m_{4 \ell}\right),
\end{aligned}
$$



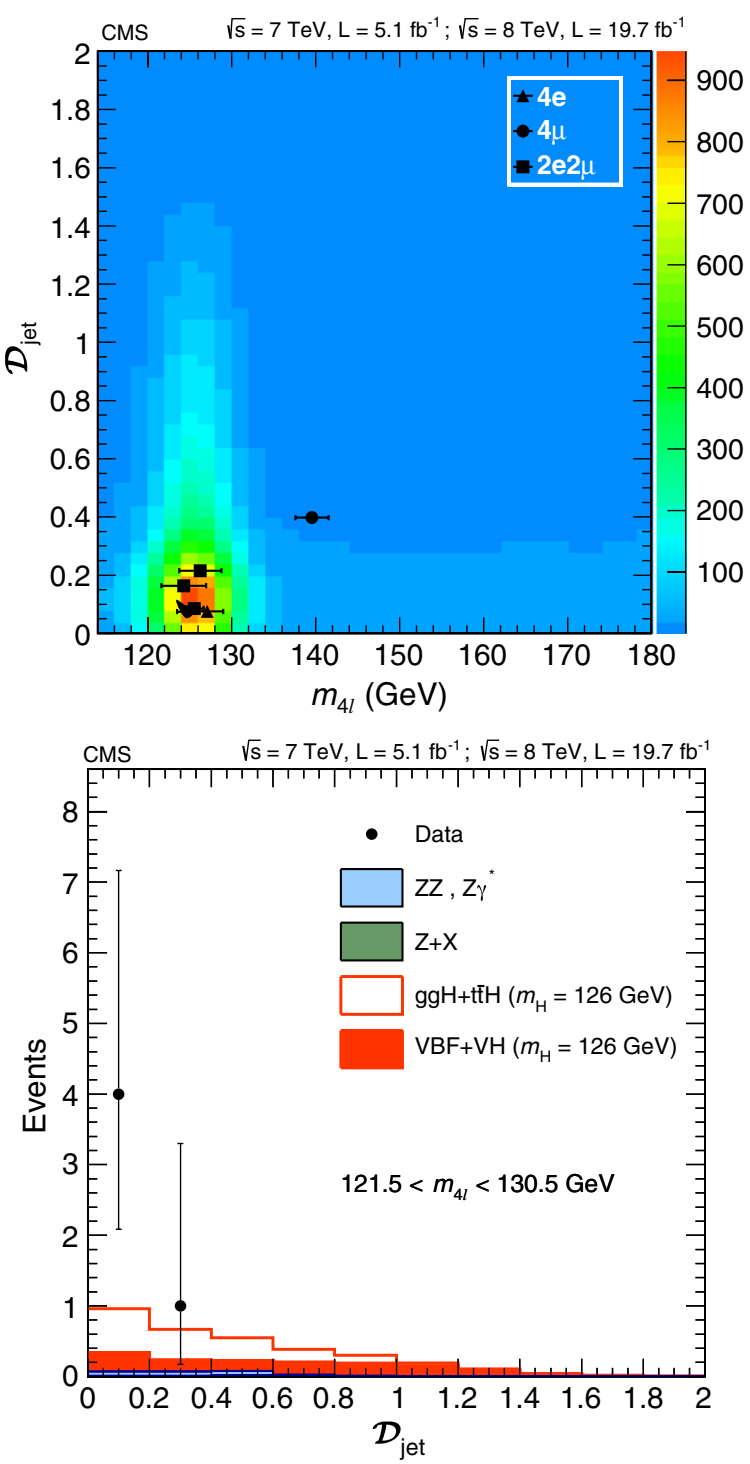

FIG. 16 (color online). (top) Distribution of $\mathscr{D}_{\text {jet }}$ versus $m_{4 \ell}$ in the low-mass-range dijet category with colors shown for the expected relative density in linear scale (in arbitrary units) of background plus the Higgs boson signal for $m_{H}=126 \mathrm{GeV}$. The points show the data, and horizontal bars represent the measured mass uncertainties. (bottom) Distribution of $\mathscr{D}_{\text {jet }}$ in the dijet category for events in the mass region $121.5<m_{4 \ell}<130.5 \mathrm{GeV}$. Points with error bars represent the data, shaded histograms represent the backgrounds, and the red histograms represent the signal expectation, broken down by production mechanism. Signal and background histograms are stacked.

where $m_{H}$ and $\Gamma$ are the mass and the width of the SM Higgs boson. The likelihood $\mathscr{L}_{3 D}^{\mu}$ includes the kinematic discriminant to differentiate the Higgs boson signal from the $Z Z$ background, defined in Eq. (7). As the third dimension of the fit, depending on the category, the production-mode-sensitive discriminant $p_{\mathrm{T}}^{4 \ell}$ of Eq. (12) (0/1-jet category) or the $\mathscr{D}_{\text {jet }}$ of Eq. (13) (dijet category) is used. These discriminants are defined in Sec. VIII. The template distributions used as probability density functions for $\mathscr{P}\left(p_{\mathrm{T}}^{4 \ell} \mid m_{4 \ell}\right)$ and $\mathscr{P}\left(\mathscr{D}_{\text {jet }} \mid m_{4 \ell}\right)$ are derived in the same way as for the $\mathscr{P}\left(\mathscr{D}_{\mathrm{bkg}}^{\mathrm{kin}} \mid m_{4 \ell}\right)$, which is discussed later in this section.

(2) For the measurement of the mass and width of the resonance, we use the following 3D likelihood:

$$
\begin{aligned}
\mathscr{L}_{3 D}^{m, \Gamma} \equiv & \mathscr{L}_{3 D}^{m, \Gamma}\left(m_{4 \ell}, \mathscr{D}_{\mathrm{m}}, \mathscr{D}_{\mathrm{bkg}}^{\mathrm{kin}}\right) \\
= & \mathscr{P}\left(m_{4 \ell} \mid m_{H}, \Gamma, \mathscr{D}_{\mathrm{m}}\right) \mathscr{P}\left(\mathscr{D}_{\mathrm{m}} \mid m_{4 \ell}\right) \\
& \times \mathscr{P}\left(\mathscr{D}_{\mathrm{bkg}}^{\mathrm{kin}} \mid m_{4 \ell}\right) .
\end{aligned}
$$

In this case, the information about the per-event mass uncertainty, $\mathscr{D}_{\mathrm{m}}$, based on the estimated resolution of the single leptons, as described in Sec. VIII A, is used. The probability density function $\mathscr{P}\left(\mathscr{D}_{\mathrm{m}} \mid m_{H}\right)$ is used for the simulated signal, while $\mathscr{P}\left(\mathscr{D}_{\mathrm{m}} \mid m_{4 \ell}\right)$ is used for backgrounds. The parameterization of the $\mathscr{P}\left(\mathscr{D}_{\mathrm{m}} \mid m_{H}\right)$ and $\mathscr{P}\left(\mathscr{D}_{\mathrm{m}} \mid m_{4 \ell}\right)$ probability density functions is discussed later in Sec. XIII B.

(3) For the spin-parity hypothesis tests, the following two-dimensional (2D) likelihood is used:

$$
\mathscr{L}_{2 D}^{J^{P}} \equiv \mathscr{L}_{2 D}^{J^{P}}\left(\mathscr{D}_{\mathrm{bkg}}, \mathscr{D}_{J^{P}}\right)
$$

In this case, as described in Sec. X, the four-lepton invariant mass and the separation of the Higgs boson signal from the $Z Z$ background using angular variables are condensed in a single discriminant, $\mathscr{D}_{\mathrm{bkg}}$, defined in Eq. (8). The second dimension of the likelihood provides discrimination between the SM Higgs boson $\left(0^{+}\right)$and the alternative $J^{P}$ hypothesis. The discriminant $\mathscr{D}_{J^{P}}$ is defined in Eq. (9). In the case of production-independent hypothesis tests, $\mathscr{D}_{\mathrm{bkg}}^{\mathrm{dec}}$ and $\mathscr{D}_{J^{P}}^{\text {dec }}$ are used.

As mentioned in Sec. III, the theoretical line shape is described by the functional form of a relativistic BW function centered at $m_{H}$ and with the expected natural width for the SM Higgs boson, $\Gamma_{H}$, in the mass region $m_{H}<400 \mathrm{GeV}$. The BW function is convolved with a double-sided CB function (to account for the core and for the asymmetric non-Gaussian tails of the experimental resolution) to parameterize the reconstructed signal $m_{4 \ell}$ distributions, $\mathscr{P}\left(m_{4 \ell} \mid m_{H}, \Gamma\right)$. The expected four-lepton mass distributions with their parameterizations superimposed for the three final states are shown in Fig. 17 for the SM Higgs boson with $m_{H}=126 \mathrm{GeV}$. For a SM Higgs boson with mass $m_{H} \geq 400 \mathrm{GeV}$, the total width is much larger than the experimental four-lepton mass resolution, as described in Sec. III. Given the $m_{4 \ell}$ distribution of the signal in the high-mass (HM) range, the functional form of the theoretical line shape has to be modified as follows: 


$$
f_{\mathrm{BW}}^{\mathrm{HM}}\left(m_{4 \ell} \mid m_{H}\right) \propto \frac{m_{4 \ell}}{\left(m_{4 \ell}^{2}-m_{H}^{2}\right)^{2}+m_{4 \ell}^{2} \cdot \Gamma_{\mathrm{HM}}^{2}},
$$

where the $\Gamma_{\mathrm{HM}}$ parameter is left floating in the fit used to determine the signal parameterization. This modified BW function is convolved with a double-sided $\mathrm{CB}$ function to account for the experimental resolution as in the low-mass case. In the fit used to determine the $m_{4 \ell}$ parameterization for $m_{H} \geq 400 \mathrm{GeV}$, the constraint that the experimental resolution parameter, $\sigma_{\mathrm{dCB}}$, must be much smaller than the natural Higgs boson width is imposed. Systematics on the line shape are incorporated by varying the signal weights for the interference effects, as a function of the generated Higgs boson mass, by $\pm 1 \sigma$.

The probability distribution $\mathscr{P}\left(m_{4 \ell}\right)$ for the background is parameterized with empirical functions using simulation for the $Z Z$ background and data control regions for the $Z+X$ background.

The correlated three-dimensional likelihood $\mathscr{L}_{3 D}^{\mu}$, defined in Eqs. (12) and (13) for the 0/1-jet and dijet categories, respectively, is described by the onedimensional (1D) parametric probability distribution $\mathscr{P}\left(m_{4 \ell}\right)$ multiplied by a two-dimensional template distribution of $\left(m_{4 \ell}, \mathscr{D}_{\mathrm{bkg}}^{\mathrm{kin}}\right)$, and a two-dimensional $\left(m_{4 \ell}, p_{\mathrm{T}}^{4 \ell}\right)$ or $\left(m_{4 \ell}, \mathscr{D}_{\text {jet }}\right)$ template distribution, where $p_{\mathrm{T}}^{4 \ell}$ is used in the $0 / 1$-jet category and $\mathscr{D}_{\text {jet }}$ is used in the dijet category. The $\mathscr{P}\left(m_{4 \ell}, \mathscr{D}_{\mathrm{bkg}}^{\mathrm{kin}}\right), \mathscr{P}\left(m_{4 \ell}, p_{\mathrm{T}}^{4 \ell}\right)$, and $\mathscr{P}\left(m_{4 \ell}, \mathscr{D}_{\text {jet }}\right)$ probabilities are normalized to 1 in the second dimension for each bin of $m_{4 \ell}$.

For the signal and background, the 2D probability density functions $\mathscr{P}\left(\mathscr{D}_{\mathrm{bkg}}^{\mathrm{kin}} \mid m_{4 \ell}\right)$ are obtained from simulation, for each of the four-lepton final states and two center-of-mass energies. The effect of instrumental uncertainties (lepton reconstruction efficiency and momentum resolution) on the shapes of this parameterization is incorporated using alternative distributions or Gaussian nuisance parameters in the likelihood and is small. For the reducible background, the probability density function is built using the control regions. The reducible background templates are found to be similar to the ones of the $q \bar{q} \rightarrow Z Z$ background. The difference in shapes is taken as a systematic uncertainty in the reducible background templates. The binning used for $\mathscr{P}\left(\mathscr{D}_{\mathrm{bkg}}^{\mathrm{kin}} \mid m_{4 \ell}\right)$ is shown in Figs. 12 (top) and 12 (bottom) for the low- and high-mass regions, respectively.

The template distributions for $\mathscr{P}\left(p_{\mathrm{T}}^{4 \ell} \mid m_{4 \ell}\right)$ are derived from simulation for both the signal and SM ZZ processes and from control regions for the $Z+X$ background. The Higgs boson $p_{\mathrm{T}}^{H}$ spectrum for gluon fusion production is obtained by tuning the POWHEG simulation to include contributions up to NNLO and NNLL expectations, including effects from resummation [137-139]. For the $p_{T}^{H}$ spectra for VBF production and the $Z Z$ background, POWHEG is used. Several uncertainties are taken into account for the probability density function $\mathscr{P}\left(p_{\mathrm{T}}^{4 \ell} \mid m_{4 \ell}\right)$ : using alternative PDF sets and varying the fixed-order QCD scales produce systematic uncertainties for all the samples. For gluon fusion Higgs boson production, variations of the default scale for NNLL resummation, and of the quark mass effects, are also considered. For the associated production process, the LO spectrum predicted by PYTHIA is used, and the difference due to NLO effects is considered as a systematic uncertainty. For the $q \bar{q} \rightarrow Z Z$ process, a systematic uncertainty is extracted, comparing the $p_{\mathrm{T}}^{Z}$ distribution of the inclusive $Z$-boson production in events simulated with POWHEG and in the data. The binning used for the $\mathscr{P}\left(p_{\mathrm{T}}^{4 \ell} \mid m_{4 \ell}\right)$ template is shown in Fig. 14 (top) for the low-mass region.

The template distributions for $\mathscr{P}\left(\mathscr{D}_{\text {jet }} \mid m_{4 \ell}\right)$ are taken from POWHEG simulations for both the signal and SM ZZ processes and from control regions for the $Z+X$ background. Alternative shapes are introduced to account for statistical and systematic uncertainties in these observables. In the dijet category, alternative shapes of $\mathscr{D}_{\text {jet }}$ arise from
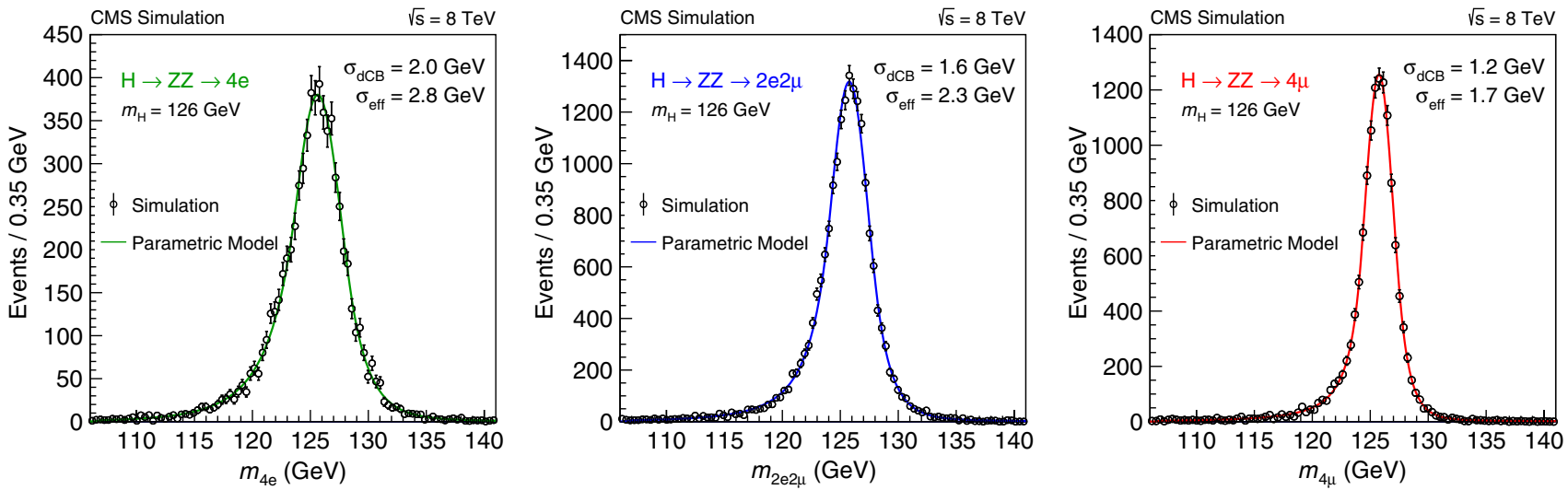

FIG. 17 (color online). The $H \rightarrow Z Z \rightarrow 4 \ell$ invariant mass distribution for $m_{H}=126 \mathrm{GeV}$ in the (left) $4 e$, (center) $2 e 2 \mu$, and (right) $4 \mu$ channels. The distributions are fitted with a double-sided CB function, and the fitted values of the CB width $\sigma_{\mathrm{dCB}}$ are indicated. The values of effective resolution, defined as half the smallest width that contains $68.3 \%$ of the distribution, are also indicated. The distributions are arbitrarily normalized. 
the comparison with different generators and underlying event tunes. The change in the $\mathscr{D}_{\text {jet }}$ shape with variations of the jet energy scale is negligible. The binning used for the $\mathscr{P}\left(\mathscr{D}_{\text {jet }} \mid m_{4 \ell}\right)$ template is shown in Fig. 16 (top) for the low-mass region.

\section{B. Systematic uncertainties}

Experimental systematic uncertainties in the normalization of the signal and the irreducible background processes are evaluated from data for the trigger, which contributes $1.5 \%$, and for the combined lepton reconstruction, identification, and isolation efficiencies, which vary from $5.5 \%$ to $11 \%$ in the $4 e$ channel, and from $2.9 \%$ to $4.3 \%$ in the $4 \mu$ channel, depending on the considered $m_{H}$. The theoretical uncertainties in the irreducible background are computed as functions of $m_{4 \ell}$, varying both the renormalization and factorization scales and the PDF set following the PDF4LHC recommendations [73,140-143]. Depending on the four-lepton mass range, the theoretical uncertainties for $q \bar{q} \rightarrow Z Z$ and $g g \rightarrow Z Z$ are $4 \%-14 \%$ and $25 \%-50 \%$, respectively.

Samples of $Z \rightarrow \ell^{+} \ell^{-}, \Upsilon(\mathrm{nS}) \rightarrow \ell^{+} \ell^{-}$, and $J / \psi \rightarrow$ $\ell^{+} \ell^{-}$events are used to set and validate the absolute momentum scale and resolution. For electrons, a $p_{\mathrm{T}}^{e}$ dependence of the momentum scale is observed, but it only marginally affects the four-lepton mass, and the perelectron uncertainty is propagated, accounting for the correlations, to the $4 e$ and $2 e 2 \mu$ channels. This dependence is corrected for, but the observed deviation is conservatively used as a systematic uncertainty, resulting in effects of $0.3 \%$ and $0.1 \%$ on the mass scales of the two channels, respectively. The systematic uncertainty in the muon momentum scale translates into a $0.1 \%$ uncertainty in the $4 \mu$ mass scale. The effect of the energy resolution uncertainties is taken into account by introducing a $20 \%$ uncertainty in the simulated width of the signal mass peak, according to the maximum deviation between data and simulation observed in the $Z \rightarrow \ell^{+} \ell^{-}$events, as shown in Fig. 3.

Additional systematic uncertainties arise from the limited statistical precision in the reducible background control regions, as well as from the difference in background composition between the control regions and the sample from which the lepton misidentification probability is derived. As described in Sec. IX, systematic uncertainties of $20 \%, 25 \%$, and $40 \%$ are assigned to the normalization of the reducible background for the $4 e, 2 e 2 \mu$, and $4 \mu$ final states, respectively. All reducible background sources are derived from control regions, and the comparison of data with the background expectation in the signal region is independent of the uncertainty in the LHC integrated luminosity of the data sample. The uncertainty in the luminosity measurement $(2.2 \%$ at $7 \mathrm{TeV}$ and $2.6 \%$ at $8 \mathrm{TeV})[144,145]$ enters the evaluation of the ZZ background and the calculation of the cross-section limit through the normalization of the signal.

Systematic uncertainties in the Higgs boson cross section and branching fraction are taken from Refs. $[55,146]$. In the $0 / 1$-jet category, an additional systematic uncertainty in the ZZ background normalization comes from the comparison of POWHEG and MADGRAPH. In the dijet category, a 30\% normalization uncertainty is taken into account for the $g g \rightarrow H+2$ jets signal cross section, while $10 \%$ is retained for the VBF production cross section. Table VI shows the summary of the systematic uncertainties in the normalization of the signal and background processes.

Shape uncertainties for both categories are considered, accounting for the lepton scale and resolution variations on the $m_{4 \ell}$ line shape, theoretical uncertainties in the $p_{\mathrm{T}}^{4 \ell}$ signal and background models, and theoretical and experimental uncertainties (such as the variations on the jet energy scale and resolution) in the $\mathscr{D}_{\text {jet }}$ distribution.

TABLE VI. Effect of systematic uncertainties on the yields of signal $\left(m_{H}=126 \mathrm{GeV}\right)$ and background processes for the $8 \mathrm{TeV}$ data set and the 0/1-jet category. Uncertainties appearing on the same line are $100 \%$ correlated, with two exceptions: those related to the missing higer orders are not correlated, and those from the $\alpha_{S}+\mathrm{PDG}(\mathrm{gg})$ in $t \bar{t} H$ are $100 \%$ anticorrelated. Uncertainties for the $7 \mathrm{TeV}$ data set are similar.

\begin{tabular}{|c|c|c|c|c|c|c|c|}
\hline \multirow[b]{2}{*}{ Source } & \multicolumn{4}{|c|}{ Signal $\left(m_{H}=126 \mathrm{GeV}\right)$} & \multicolumn{3}{|c|}{ Backgrounds } \\
\hline & $g g H$ & VBF & $V H$ & $t \bar{t} H$ & $q \bar{q} \rightarrow Z Z$ & $g g \rightarrow Z Z$ & $Z+X$ \\
\hline$\alpha_{S}+\mathrm{PDF}(\mathrm{gg})$ & $7.2 \%$ & $\cdots$ & $\cdots$ & $7.8 \%$ & $\ldots$ & $7.2 \%$ & $\cdots$ \\
\hline$\alpha_{S}+\operatorname{PDF}(q \bar{q})$ & $\ldots$ & $2.7 \%$ & $3.5 \%$ & $\ldots$ & $3.4 \%$ & $\ldots$ & $\cdots$ \\
\hline Missing higher orders & $7.5 \%$ & $0.2 \%$ & $0.4 \%, 1.6 \%$ & $6.6 \%$ & $2.9 \%$ & $24 \%$ & $\cdots$ \\
\hline Signal acceptance & & & $2 \%$ & & $\ldots$ & $\cdots$ & $\cdots$ \\
\hline $\mathrm{BR}(H \rightarrow Z Z)$ & & & $2 \%$ & & $\cdots$ & $\cdots$ & $\cdots$ \\
\hline Luminosity & \multicolumn{6}{|c|}{$2.6 \%$} & $\cdots$ \\
\hline Electron efficiency & \multicolumn{6}{|c|}{$10 \%(4 e), 4.3 \%(2 e 2 \mu)$} & $\cdots$ \\
\hline Muon efficiency & \multicolumn{6}{|c|}{$4.3 \%(4 \mu), 2.1 \%(2 e 2 \mu)$} & $\cdots$ \\
\hline Control region & $\cdots$ & $\cdots$ & $\ldots$ & $\cdots$ & $\cdots$ & $\cdots$ & $40 \%$ \\
\hline
\end{tabular}




\section{RESULTS AND INTERPRETATION}

The results of the search for a signal consistent with a SM Higgs boson in the $m_{H}$ range $110-1000 \mathrm{GeV}$ are described along with the estimation of the significance of the excess observed in the low-mass region. Then, the measurement of the mass of the new boson in the hypothesis of a narrow resonance and limits on its width are reported. For this resonance, the compatibility of the cross section measurement with the SM Higgs boson calculation is given together with constraints on the production mechanisms. Finally, the spin and parity of the boson are tested to check the compatibility with the hypothesis of a $0^{+}$resonance as compared with the alternatives, and the measurement of the fraction of a $\mathrm{CP}$-odd contribution to the decay amplitude is reported.

\section{A. Signal significance and exclusion limits}

The selected events are split into twelve subcategories based on the three final states, two data-taking periods ( 7 and $8 \mathrm{TeV}$ ), and two jet categories. These events are examined for 187 hypothetical SM-like Higgs boson masses in a range between 110 and $1000 \mathrm{GeV}$, where the mass steps are optimized to account for the expected width and resolution [136]. A 3D model, $\mathscr{L}_{3 D}^{\mu, 0 / 1-\mathrm{jet}}\left(m_{4 \ell}, \mathscr{D}_{\mathrm{bkg}}^{\mathrm{kin}}, p_{\mathrm{T}}^{4 \ell}\right)$ and $\mathscr{L}_{3 D}^{\mu \text {,dijet }}\left(m_{4 \ell}, \mathscr{D}_{\mathrm{bkg}}^{\mathrm{kin}}, \mathscr{D}_{\text {jet }}\right)$, defined, respectively, in Eqs. (12) and (13) for the 0/1-jet category and for the dijet category, is used. The statistical approach discussed in Ref. [136] is followed to set exclusion limits and to establish the significance of an excess. The modified frequentist construction $\mathrm{CL}_{s}[136,147,148]$ is adopted as the primary method for reporting limits. As a complementary method to the frequentist construction, a Bayesian approach [149] yields consistent results.

Upper limits on the ratio of the production cross section to the SM expectation are shown in Fig. 18 (top). The results presented in this section make use of asymptotic formulas from Ref. [150]. The SM-like Higgs boson is excluded by the four-lepton channels at the 95\% C.L. in the mass ranges $114.5-119.0 \mathrm{GeV}$ and $129.5-832.0 \mathrm{GeV}$, for an expected exclusion range of $115-740 \mathrm{GeV}$. The local $p$ values, representing the significance of a local excess relative to the background expectation, are shown for the full mass range as a function of $m_{H}$ in Fig. 18 (bottom). The minimum of the local $p$ value is reached around $m_{4 \ell}=125.7 \mathrm{GeV}$, near the mass of the new boson, confirming the result in Ref. [20], and corresponds to a local significance of $6.8 \sigma$, consistent with the expected sensitivity of $6.7 \sigma$. As a cross-check, 1D $\left[\mathscr{L}_{1 D}^{\mu} \equiv\right.$ $\left.\mathscr{L}_{1 D}^{\mu}\left(m_{4 \ell}\right)\right]$ and 2D $\left[\mathscr{L}_{2 D}^{\mu} \equiv \mathscr{L}_{2 D}^{\mu}\left(m_{4 \ell}, \mathscr{D}_{\mathrm{bkg}}^{\mathrm{kin}}\right)\right]$ models are also studied, as shown in Figs. 18 (bottom) and 19, resulting in an observed local significance of $5.0 \sigma$ and $6.9 \sigma$, for an expectation of $5.6 \sigma$ and $6.6 \sigma$, respectively. These results are consistent with the 3D model; however, with a systematically lower expected sensitivity to the signal. No other significant deviations with respect to the expectations is found in the mass range $110-1000 \mathrm{GeV}$. The second most significant $p$-value minimum is reached around $m_{4 \ell}=146 \mathrm{GeV}$, with a local significance of $2.7 \sigma$. This computation does not take into account the lookelsewhere effect [151].

\section{B. Mass and width}

In order to measure the mass and width of the new boson precisely and to correctly assign the uncertainties in these measurements, the four-lepton mass uncertainties estimated on a per-event basis, as described in Sec. VIII A, are incorporated into the likelihood. This approach has the largest impact in a context of a low number of events and a wide spread of per-event uncertainties, both of which are present in the $H \rightarrow Z Z \rightarrow 4 \ell$ analysis. Tests on simulation indicate that, with this approach, the uncertainties in the measured mass and the upper limit on the width of the Higgs boson are expected to improve by about $8 \%$ and $10 \%$, respectively, with respect to using the average resolution.

The experimental resolution parameter of the doublesided CB function, used to model the $m_{4 \ell}$ line shape, is substituted with the per-event estimation of the mass uncertainty $\mathscr{D}_{\mathrm{m}}$. The parameters describing the tail of the double-sided CB from simulation are also corrected on a per-event basis.

The likelihood used for the mass and width measurements is defined in Eq. (14). By construction, this likelihood neglects potential correlations between $\mathscr{D}_{\mathrm{bkg}}^{\mathrm{kin}}$ and $\mathscr{D}_{\mathrm{m}}$. Simulated Higgs boson and $q \bar{q} \rightarrow Z Z$ events show no evident correlations between these two observables. The probability density functions $\mathscr{P}\left(\mathscr{D}_{\mathrm{m}} \mid m_{H}\right)$ of the per-event uncertainty distributions for the signal are obtained from simulation. The probability density functions $\mathscr{P}\left(\mathscr{D}_{\mathrm{m}} \mid m_{4 \ell}\right)$ for the $Z Z$ background are obtained from simulation and are cross-checked with data in control regions dominated by the $Z Z$ background events $\left(m_{4 \ell}>180 \mathrm{GeV}\right)$ and $Z \rightarrow 4 \ell$ events $\left(80<m_{4 \ell}<100 \mathrm{GeV}\right)$ [152], as shown in Fig. 6 (bottom). The $\mathscr{P}\left(\mathscr{D}_{\mathrm{m}} \mid m_{4 \ell}\right)$ for the reducible background is obtained from the control regions in the data with the same technique used to derive the $m_{4 \ell}$ line shapes. The $\mathscr{P}\left(\mathscr{D}_{\mathrm{m}} \mid m_{4 \ell}\right)$ is a conditional probability distribution function, where for all the channels and both signal and background components, the probability density functions $\mathscr{P}\left(\mathscr{D}_{\mathrm{m}}\right)$ are parameterized as a sum of a Landau [108] and a Gaussian function.

Figure 20 (top) shows the profile likelihood scan versus the SM Higgs boson mass performed under the assumption that its width is much smaller than the detector resolution, for the single channels, combining 7 and $8 \mathrm{TeV}$ data, and for the combination of all the channels. The Higgs boson cross section is left floating in the fit. To decompose the total mass uncertainty into statistical and systematic components, a fit with all nuisance parameters fixed at their 

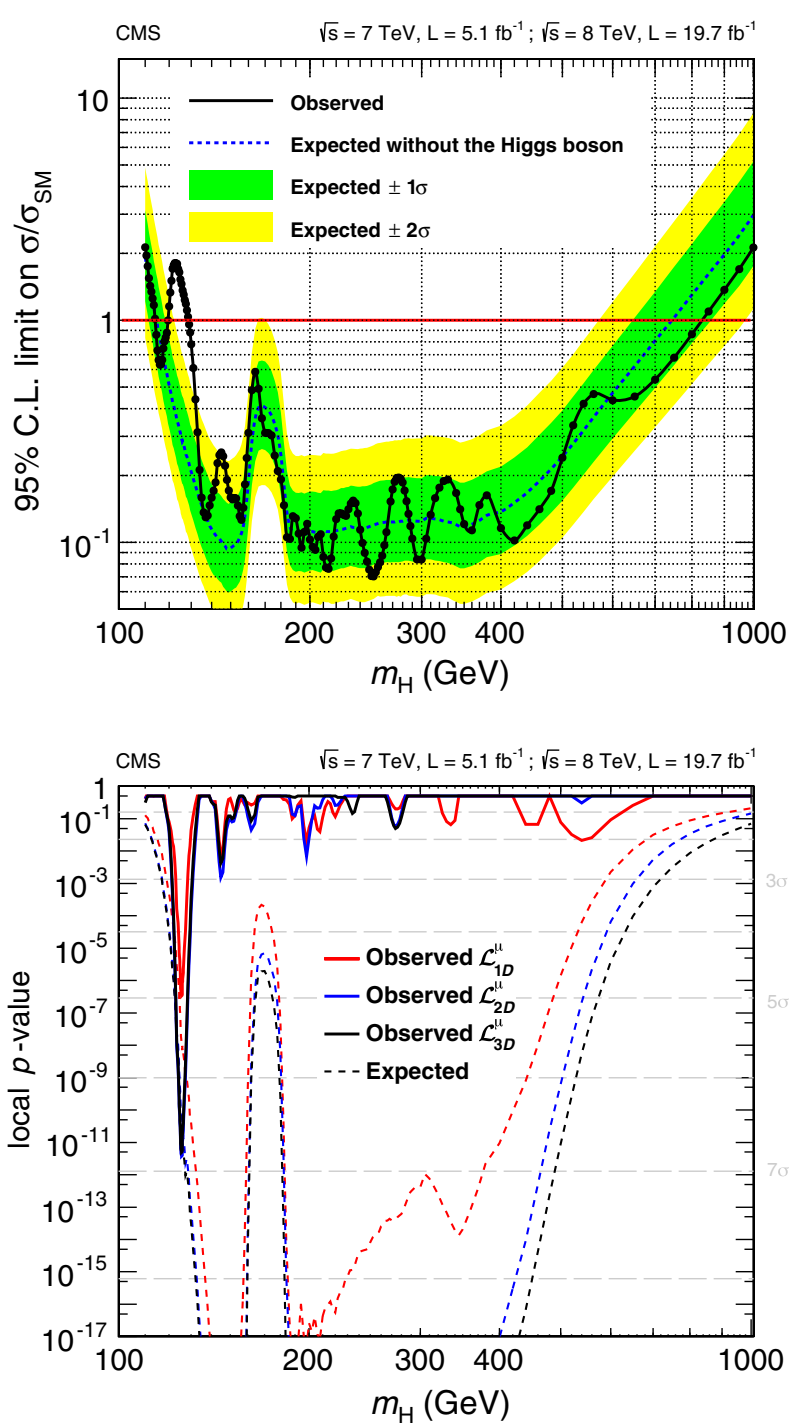

FIG. 18 (color online). (top) Observed and expected 95\% C.L. upper limit on the ratio of the production cross section to the SM expectation. The expected $1 \sigma$ and $2 \sigma$ ranges of expectation for the background-only model are also shown with green and yellow bands, respectively. (bottom) Significance of the local excess with respect to the SM background expectation as a function of the Higgs boson mass in the full mass range $110-1000 \mathrm{GeV}$. Results are shown for the $1 \mathrm{D}$ fit $\left(\mathscr{L}_{1 D}^{\mu}\right)$, the $2 \mathrm{D}$ fit $\left(\mathscr{L}_{2 D}^{\mu}\right)$, and the reference $3 \mathrm{D}$ fit $\left(\mathscr{L}_{3 D}^{\mu}\right)$.

best-fit values is performed. The mass uncertainty obtained in this way is purely statistical. The systematic uncertainties account for an effect on the mass scale of the lepton momentum scale and resolution, shape systematics in the $\mathscr{P}\left(\mathscr{D}_{\mathrm{bkg}}^{\mathrm{kin}} \mid m_{4 \ell}\right)$ probability density functions used as signal and background models, and normalization systematics due to acceptance and efficiency uncertainty. The measured mass is $m_{H}=125.6 \pm 0.4$ (stat) \pm 0.2 (syst) $\mathrm{GeV}$.

Figure 20 (top) also shows likelihood scans separately for the $4 e, 2 e 2 \mu$, and $4 \mu$ final states when using the 3D model $\mathscr{L}_{3 D}^{m, \Gamma}$ of Eq. (14). The measurements in the three

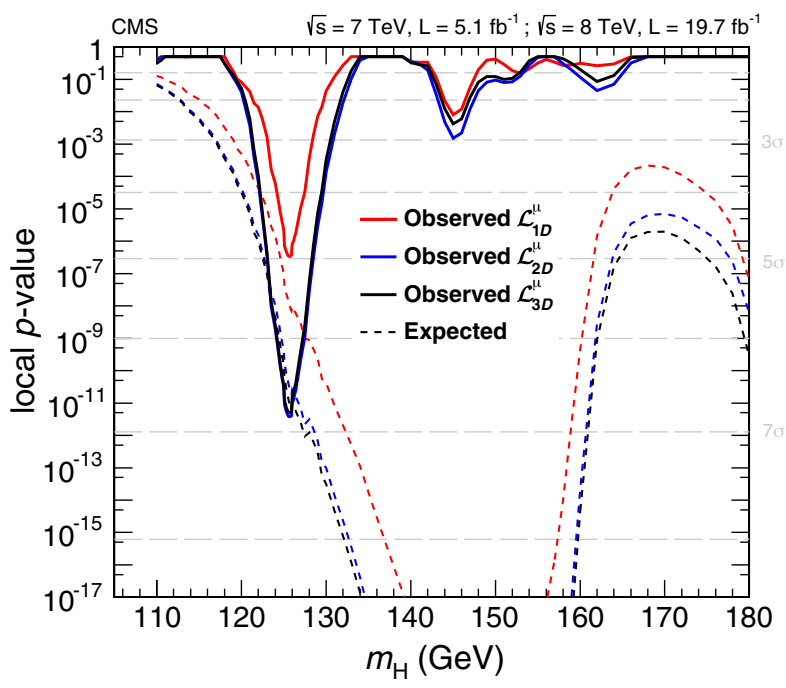

FIG. 19 (color online). Significance of the local excess with respect to the SM background expectation as a function of the Higgs boson mass for the $1 \mathrm{D}$ fit $\left(\mathscr{L}_{1 D}^{\mu}\right)$, the $2 \mathrm{D}$ fit $\left(\mathscr{L}_{2 D}^{\mu}\right)$, and the reference $3 \mathrm{D}$ fit $\left(\mathscr{L}_{3 D}^{\mu}\right)$. Results are shown for the full data sample in the low-mass region only.

final states are statistically compatible. The best-fit values for each subchannel are also shown in Table VII. The dominant contribution to the systematic uncertainty is the limited knowledge of the lepton momentum scale.

Two more mass measurements are performed with a reduced level of information, by dropping the $\mathscr{P}\left(\mathscr{D}_{\mathrm{bkg}}^{\mathrm{kin}} \mid m_{4 \ell}\right)$ term of the likelihood in Eq. (14), resulting in a 2D model, $\mathscr{L}_{2 D}^{m, \Gamma} \equiv \mathscr{L}_{2 D}^{m, \Gamma}\left(m_{4 \ell}, \mathscr{D}_{\mathrm{m}}\right)$, or by performing only a mass line shape fit and assuming the average mass resolution is applicable for each channel, resulting in a 1D model, $\mathscr{L}_{1 D}^{m, \Gamma} \equiv \mathscr{L}_{1 D}^{m, \Gamma}\left(m_{4 \ell}\right)$. The measured central value is the same in all three cases, with an increasing uncertainty, due to the reduced information available to the fit in the case of $2 \mathrm{D}$ or 1D models. Figure 20 (right) shows the likelihood scans for the combination of all the final states separately for the $\mathscr{L}_{1 D}^{m, \Gamma}, \mathscr{L}_{2 D}^{m, \Gamma}$, and $\mathscr{L}_{3 D}^{m, \Gamma}$ models.

The mass distribution for the $Z \rightarrow 4 \ell$ decay exhibits a pronounced resonant peak at $m_{4 \ell}=m_{Z}$ close to the new boson $\left(80<m_{4 \ell}<100 \mathrm{GeV}\right)$. Hence, the $Z \rightarrow 4 \ell$ peak can be used as validation of the measurement of the mass of the new boson using the same techniques as for the Higgs boson. The mass of the reconstructed $Z$ boson in $Z \rightarrow 4 \ell$ decays, with the assumption of the Particle Data Group (PDG) [149] value for the Z-boson natural width, is consistent in each subchannel. The measured value for the combination of all the $Z \rightarrow 4 \ell$ final states is $m_{Z}=$ $91.1 \mathrm{GeV}$, compatible with the PDG value $(91.1876 \pm$ $0.0021 \mathrm{GeV}$ ) within the total estimated uncertainty of $0.4 \mathrm{GeV}$ [149].

Figure 21 shows the scan of the 3D likelihood versus the width of the SM-like Higgs boson with an arbitrary width. In this scan, the mass and the signal strength $\mu$ are profiled, as all other nuisance parameters. This shows that the data 

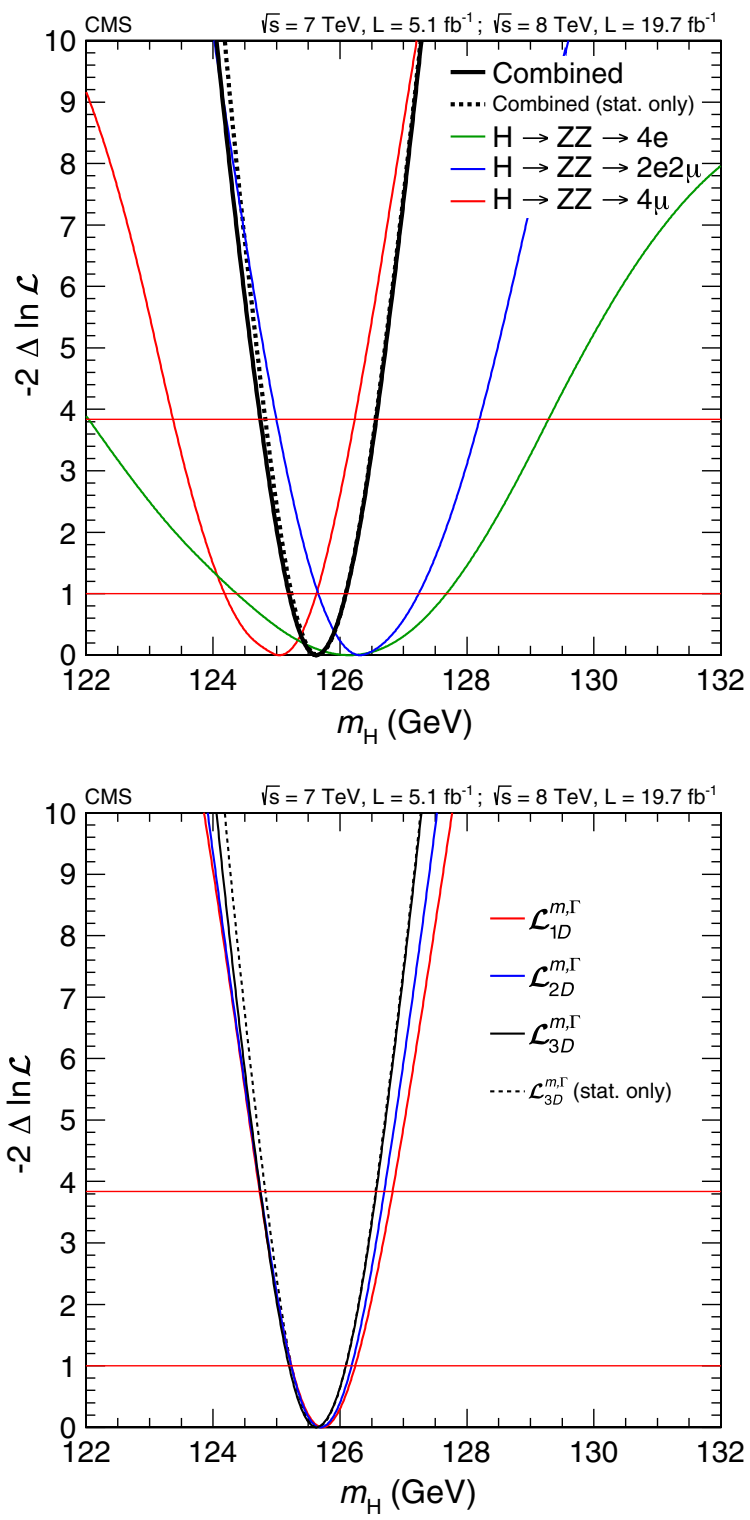

FIG. 20 (color online). (top) Scan of the negative log likelihood $-2 \Delta \ln \mathscr{L}$ versus the SM Higgs boson mass $m_{H}$, for each of the three channels separately and the combination of the three, where the dashed line represents the scan including only statistical uncertainties when using the 3D model. (bottom) Scan of $-2 \Delta \ln \mathscr{L}$ versus $m_{H}$ for the combination of the three channels, and using the $1 \mathrm{D}$ fit $\left(\mathscr{L}_{1 D}^{m, \Gamma}\right), 2 \mathrm{D}$ fit $\left(\mathscr{L}_{2 D}^{m, \Gamma}\right)$, and $3 \mathrm{D}$ fit $\left(\mathscr{L}_{3 D}^{m, \Gamma}\right)$. The horizontal lines at $-2 \Delta \ln \mathscr{L}=1$ and 3.84 represent the $68 \%$ and 95\% C.L.'s, respectively.

are compatible with a narrow-width resonance. The measured width is $\Gamma_{H}=0.0_{-0.0}^{+1.3} \mathrm{GeV}$, and the upper limit on the width is $3.4 \mathrm{GeV}$ at the $95 \%$ C.L. The expected upper limit is $2.8 \mathrm{GeV}$.

\section{Signal strength}

The measured signal strength is $\mu=\sigma / \sigma_{\mathrm{SM}}=$ $0.93_{-0.23}^{+0.26}(\text { stat })_{-0.09}^{+0.13}$ (syst) at the best-fit mass $\left(m_{H}=\right.$ $125.6 \mathrm{GeV}$ ) with the models of Eqs. (12) and (13) for
TABLE VII. Best-fit values for the mass of the Higgs boson candidate, measured in the $4 \ell, \ell=e, \mu$ final states using a $\mathscr{L}_{3 D}^{m, \Gamma}$ model. For the combination of all the final states $H \rightarrow 4 \ell$, the separate contributions of the statistical and systematic uncertainty to the total one are given.

\begin{tabular}{lc}
\hline \hline Channel & Measured mass $(\mathrm{GeV})$ \\
\hline $4 e$ & $126.2_{-1.8}^{+1.5}$ \\
$2 e 2 \mu$ & $126.3_{-0.7}^{+0.9}$ \\
$4 \mu$ & $125.1_{-0.9}^{+0.6}$ \\
$4 \ell$ & $125.6 \pm 0.4$ (stat) \pm 0.2 (syst) \\
\hline \hline
\end{tabular}

the 0/1-jet category and the dijet category, respectively. The median expected signal strength is $\mu=1.00_{-0.26}^{+0.31}$, for which the total uncertainty agrees with the observed one. The result is $0.83_{-0.25}^{+0.31}$ in the $0 / 1$-jet category and $1.45_{-0.62}^{+0.89}$ in the dijet category. The best-fit values are shown in Fig. 22 (top). For each category, the signal strength is consistent with SM expectations within the uncertainties, which are dominated by the statistical ones with the current data set.

The categorization according to jet multiplicity and the inclusion of VBF-sensitive variables in the likelihood, like $p_{\mathrm{T}}^{4 \ell}$ and $\mathscr{D}_{\text {jet }}$, used to measure the cross section in the inclusive category, are also used to disentangle the production mechanisms of the observed new state. The production mechanisms are split into two families depending on whether the production is through couplings to fermions (gluon fusion, $t \bar{t} H$ ) or vector bosons (VBF, $V H$ ). For $m_{H}=126 \mathrm{GeV}$, about $55 \%$ of the VBF events are expected to be included in the dijet category, while only $8 \%$

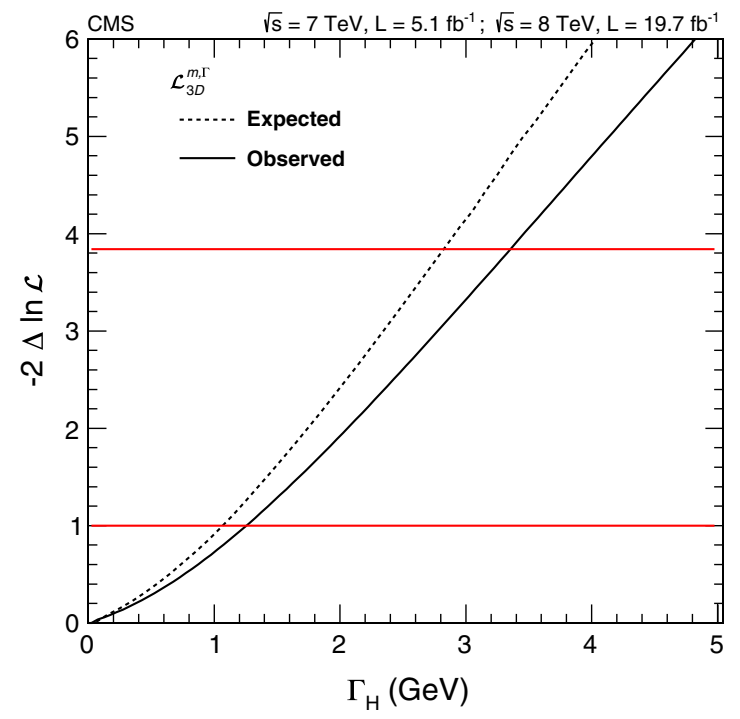

FIG. 21 (color online). Scan of the average expected and observed negative $\log$ likelihood $-2 \Delta \ln \mathscr{L}$ versus the tested SM Higgs boson width $\Gamma_{H}$ obtained with the $3 \mathrm{D}$ fit $\left(\mathscr{L}_{3 D}^{m, \Gamma}\right)$. The horizontal lines at $-2 \Delta \ln \mathscr{L}=1$ and 3.84 represent the $68 \%$ and 95\% C.L.'s, respectively. 
of the gluon fusion events are included in the dijet category. As shown in Table V, a fraction of $43 \%$ of $\mathrm{WH}$ and $\mathrm{ZH}$ production contributes to the dijet category. Events that contribute are those in which the vector boson decays hadronically.

Two signal-strength modifiers $\left(\mu_{g g H, t \bar{t} H}\right.$ and $\left.\mu_{\mathrm{VBF}, \mathrm{VH}}\right)$ are introduced as scale factors for the fermion and vectorboson induced contribution to the expected SM cross section. A two-dimensional fit is performed for the two signal-strength modifiers assuming a mass hypothesis of $m_{H}=125.6 \mathrm{GeV}$. The likelihood is profiled for all
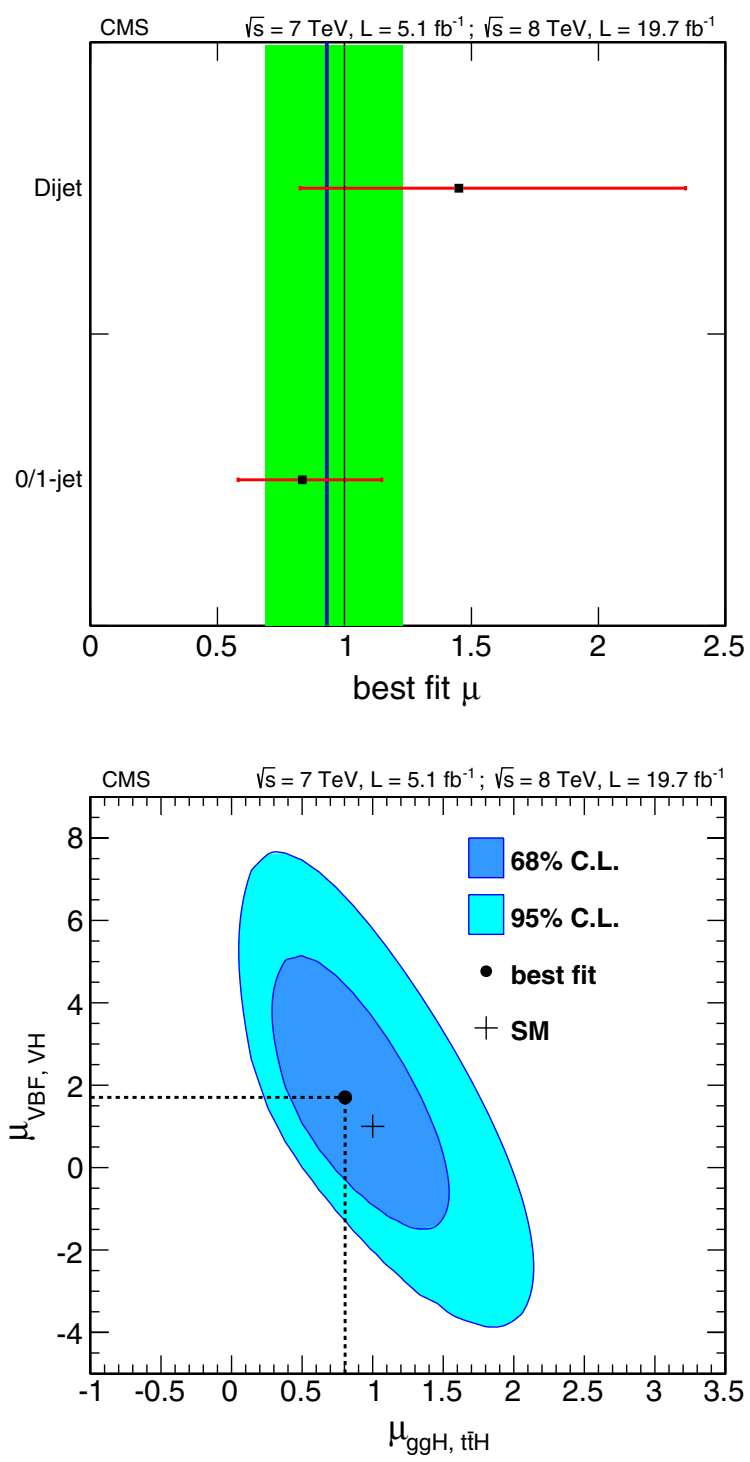

FIG. 22 (color online). (top) Values of $\mu$ for the two categories. The vertical line shows the combined $\mu$ together with its associated $\pm 1 \sigma$ uncertainties, shown as a green band. The horizontal bars indicate the $\pm 1 \sigma$ uncertainties in $\mu$ for the different categories. The uncertainties include both statistical and systematic sources of uncertainty. (bottom) Likelihood contours on the signal-strength modifiers associated with fermions $\left(\mu_{g g H, t \bar{t} H}\right)$ and vector bosons $\left(\mu_{\mathrm{VBF}, \mathrm{VH}}\right)$ shown at a $68 \%$ and $95 \%$ C.L. nuisance parameters and $68 \%$ and $95 \%$ C.L. contours in the $\left(\mu_{g g H, t \bar{t} H}, \mu_{\mathrm{VBF}, \mathrm{VH}}\right)$ plane are obtained. Figure 22 (bottom) shows the result of the fit leading to the measurements of $\mu_{g g H, t \bar{t} H}=0.80_{-0.36}^{+0.46}$ and $\mu_{\mathrm{VBF}, \mathrm{VH}}=1.7_{-2.1}^{+2.2}$. The measured values are consistent with the expectations for the SM Higgs boson, $\left(\mu_{g g H, t \bar{t} H}, \mu_{\mathrm{VBF}, \mathrm{VH}}\right)=(1,1)$. With the current limited statistics, we cannot establish yet the presence of $\mathrm{VBF}$ and $V H$ production, since $\mu_{\mathrm{VBF}, \mathrm{VH}}=0$ is also compatible with the data. Since the decay (into $Z Z$ ) is vector-boson mediated, it is necessary that such a coupling must exist in the production side and that the SM VBF and SM $V H$ production mechanisms must be present. The fitted value of $\mu_{\mathrm{VBF}, \mathrm{VH}}$ larger than 1 is driven partly by the hard $p_{\mathrm{T}}^{4 \ell}$ spectrum of the events observed in data when
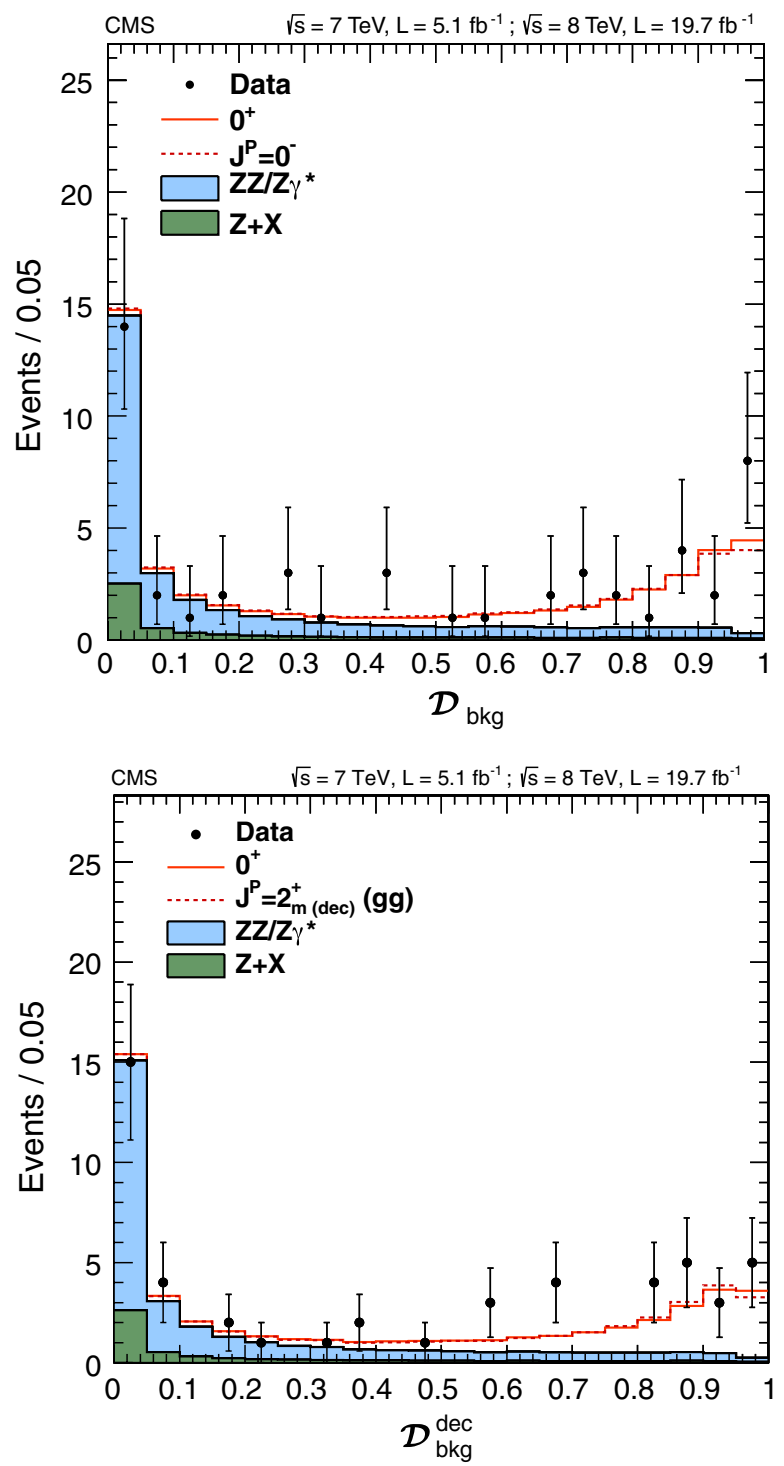

FIG. 23 (color online). Distribution of $\mathscr{D}_{\mathrm{bkg}}$ (top) and $\mathscr{D}_{\mathrm{bkg}}^{\mathrm{dec}}$ for the production-independent scenario (bottom) in data and MC expectations for the background and for a signal resonance consistent with the SM Higgs boson with $m_{0^{+}}=125.6 \mathrm{GeV}$. 
compared to the expectation from the production of the SM Higgs boson (Fig. 14).

\section{Spin and parity}

To measure the spin and parity properties of the new boson, the methodology discussed in Sec. X is followed. In addition to the models tested in Ref. [31] $\left(0^{-}\right.$and $\left.g g \rightarrow 2_{\mathrm{m}}^{+}\right)$, seven additional models are examined: $0_{\mathrm{h}}^{+}, q \bar{q} \rightarrow 1^{-}$, $q \bar{q} \rightarrow 1^{+}, q \bar{q} \rightarrow 2_{\mathrm{m}}^{+}, g g \rightarrow 2_{\mathrm{h}}^{+}, g g \rightarrow 2_{\mathrm{h}}^{-}, g g \rightarrow 2_{\mathrm{b}}^{+}$. The discrimination is based on 2D probability density functions $\left(\mathscr{D}_{\mathrm{bkg}}, \mathscr{D}_{J^{P}}\right)$, where the kinematic discriminants $\mathscr{D}_{\mathrm{bkg}}$ and $\mathscr{D}_{J^{P}}$ are defined by Eqs. (8) and (9). The $1^{ \pm}$and $2_{\mathrm{m}}^{+}$signal hypotheses are also tested by relying only on their decay information, i.e. in a production-independent way, using pairs of kinematic discriminants $\left(\mathscr{D}_{\mathrm{bkg}}^{\mathrm{dec}}, \mathscr{D}_{J^{P}}^{\mathrm{dec}}\right)$, defined by Eqs. (10) and (11). All models and discriminants, discussed in Sec. X, are listed in Table II.

For spin and parity studies, the event categorization based on jets is not used in order to reduce the dependence on the production mechanisms. Consequently, the VBF discriminants, $p_{\mathrm{T}}^{4 \ell}$ and $\mathscr{D}_{\text {jet }}$, are not used, resulting in the $\mathscr{L}_{2 D}^{J^{P}}$ model defined in Eq. (15). Events in the mass range $106<m_{4 \ell}<$ $141 \mathrm{GeV}$ are used to perform these studies. The Higgs boson mass is assumed to be $m_{0^{+}}=125.6 \mathrm{GeV}$. The $2 \mathrm{D}$ probability density functions for signal and background, $\mathscr{P}\left(\mathscr{D}_{J^{P}}, \mathscr{D}_{\text {bkg }}\right)$ in Eq. (15), are obtained as 2D templates from simulation for the signal and irreducible background, and from control regions for the reducible backgrounds.

Figure 23 shows expected and observed distributions for the discriminants $\mathscr{D}_{\mathrm{bkg}}$ and $\mathscr{D}_{\mathrm{bkg}}^{\mathrm{dec}}$. The distributions are very similar for the SM and all alternative signal hypotheses but differ significantly from the background. Figures 24 and 25 show distributions for the $\mathscr{D}_{J^{P}}$ observables for all tested signal hypotheses. Only one alternative hypothesis is shown on each figure. The distributions show events with $\mathscr{D}_{\text {bkg }}^{(\mathrm{dec})}>0.5$ to enhance the fraction of signal events for illustration purposes only. For the hypothesis tests, the full range of the discriminant is used.

The alternative signal models are defined by the tensor structure of couplings; however, the absolute values of couplings, and hence, the expected event yields are not uniquely defined. The cross sections for alternative signal hypotheses are left floating in the fit. The same approach is taken for the SM Higgs boson hypothesis: i.e., the overall SM Higgs boson signal strength $\mu$ is the best-fit value as it comes out from data. This way, the overall signal event yield is not a part of the discrimination between alternative hypotheses. Consequently, for pairwise tests of alternative signal hypotheses with respect to the SM Higgs boson, the
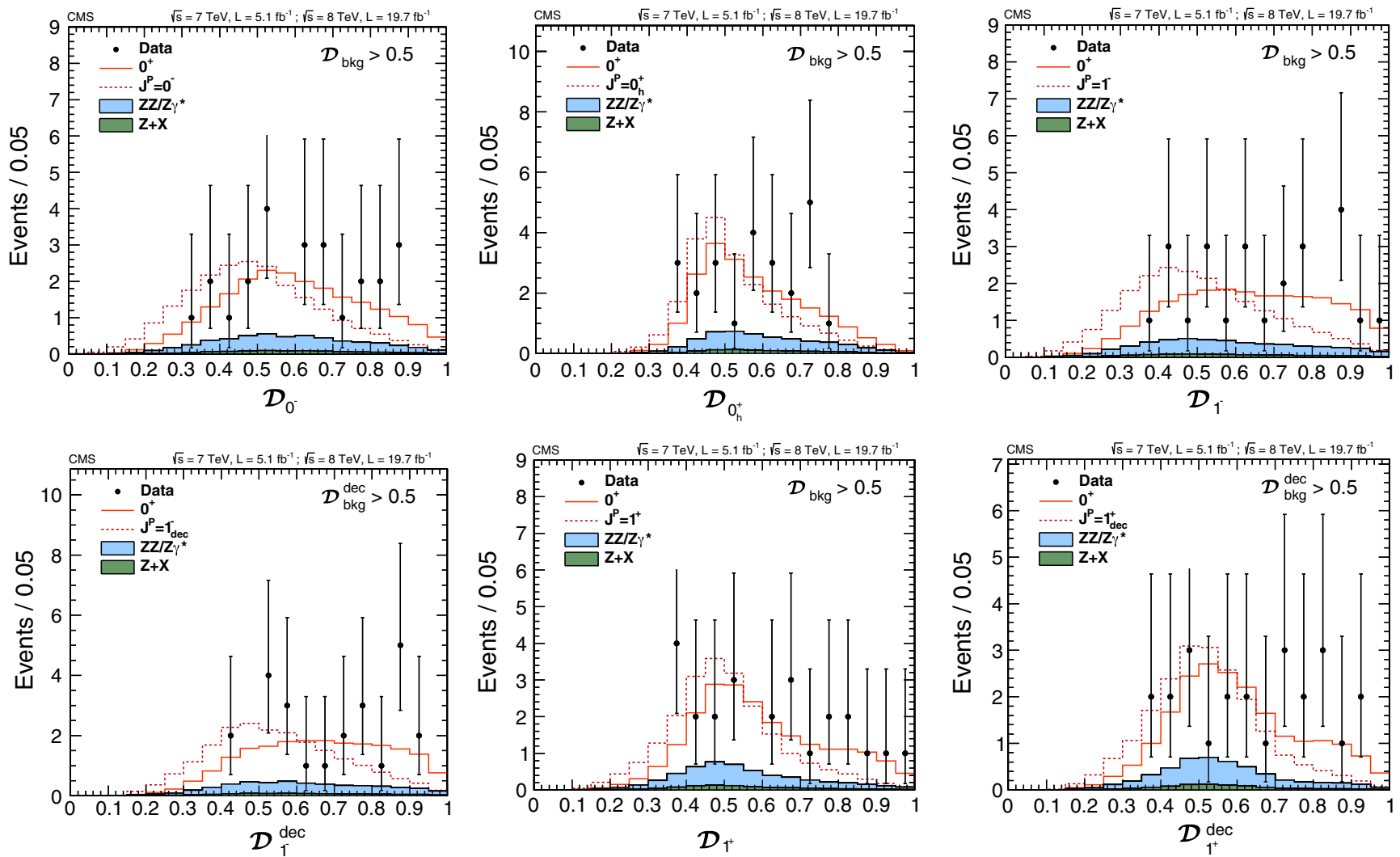

FIG. 24 (color online). Distributions of $\mathscr{D}_{J^{P}}$ with a requirement $\mathscr{D}_{\mathrm{bkg}}^{(\mathrm{dec})}>0.5$. Distributions in data (points with error bars) and expectations for background and signal are shown: six alternative $J^{P}$ hypotheses are shown. $J^{P}=0^{-}$(upper left), $0_{\mathrm{h}}^{+}$(upper middle), $1^{-}(q \bar{q})$ (upper right), $1^{-}$(lower left), $1^{+}(q \bar{q})$ (lower middle), $1^{+}$(lower right). 
test statistic is defined using the ratio of signal plus background likelihoods for two signal hypotheses $q=-2 \ln \left(\mathscr{L}_{J^{p}} / \mathscr{L}_{0^{+}}\right)$. The expected distribution of $q$ for the pseudoscalar hypothesis (blue histogram) and the SM Higgs boson (orange histogram) are shown in Fig. 26 (top). Similar distributions for the test statistic $q$ are obtained for the other alternative hypotheses considered. The pseudoexperiments are generated using the nuisance parameters fitted in data.

To quantify the consistency of the observed test statistics $q_{\mathrm{obs}}$ with respect to the SM Higgs boson hypothesis $\left(0^{+}\right)$, we assess the probability $p=P\left(q \leq q_{\mathrm{obs}} \mid 0^{+}+\right.$bkg $)$and convert it into a number of standard deviations $Z$ via the Gaussian one-sided tail integral:

$$
p=\int_{Z}^{\infty} \frac{1}{\sqrt{2 \pi}} \exp \left(-x^{2} / 2\right) \mathrm{d} x
$$

Similarly, the consistency of the observed data with alternative signal hypotheses $\left(J^{P}\right)$ is assessed from $P\left(q \geq q_{\mathrm{obs}} \mid J^{P}+\mathrm{bkg}\right)$. The $\mathrm{CL}_{s}$ criterion, defined as $\mathrm{CL}_{s}=P\left(q \geq q_{\mathrm{obs}} \mid J^{P}+\mathrm{bkg}\right) / P\left(q \geq q_{\mathrm{obs}} \mid 0^{+}+\mathrm{bkg}\right)<\alpha$, is used for the final inference of whether a particular alternative signal hypotheses is excluded or not with a given confidence level $(1-\alpha)$.
The expected separations between alternative signal hypotheses are quoted for two cases. In the first case, the expected SM Higgs boson signal strength and the alternative signal cross sections are equal to the ones obtained in the fit of the data. The second case assumes the nominal SM Higgs boson signal strength $(\mu=1$, as indicated in parentheses for expectations quoted in Table VIII), while the cross sections for the alternative signal hypotheses are taken to be the same as for the SM Higgs boson (the $2 e 2 \mu$ channel is taken as a reference). Since the observed signal strength is very close to unity, the two results for the expected separations are also similar. The observed values of the test statistic in the case of the SM Higgs boson versus a pseudoscalar boson are shown with red arrows in Fig. 26 (top). Results obtained from the test statistic distributions are summarized in Table VIII and in Fig. 27.

The observed value of the test statistic is larger than the median expected for the SM Higgs boson. This happens for many distributions because of strong kinematic correlations between different signal hypotheses, most prominently seen in the $m_{Z_{2}}$ distributions. The pseudoscalar $\left(0^{-}\right)$and all spin-1 hypotheses tested are excluded at the $99.9 \%$ or higher C.L. All tested spin-2 models are excluded at the
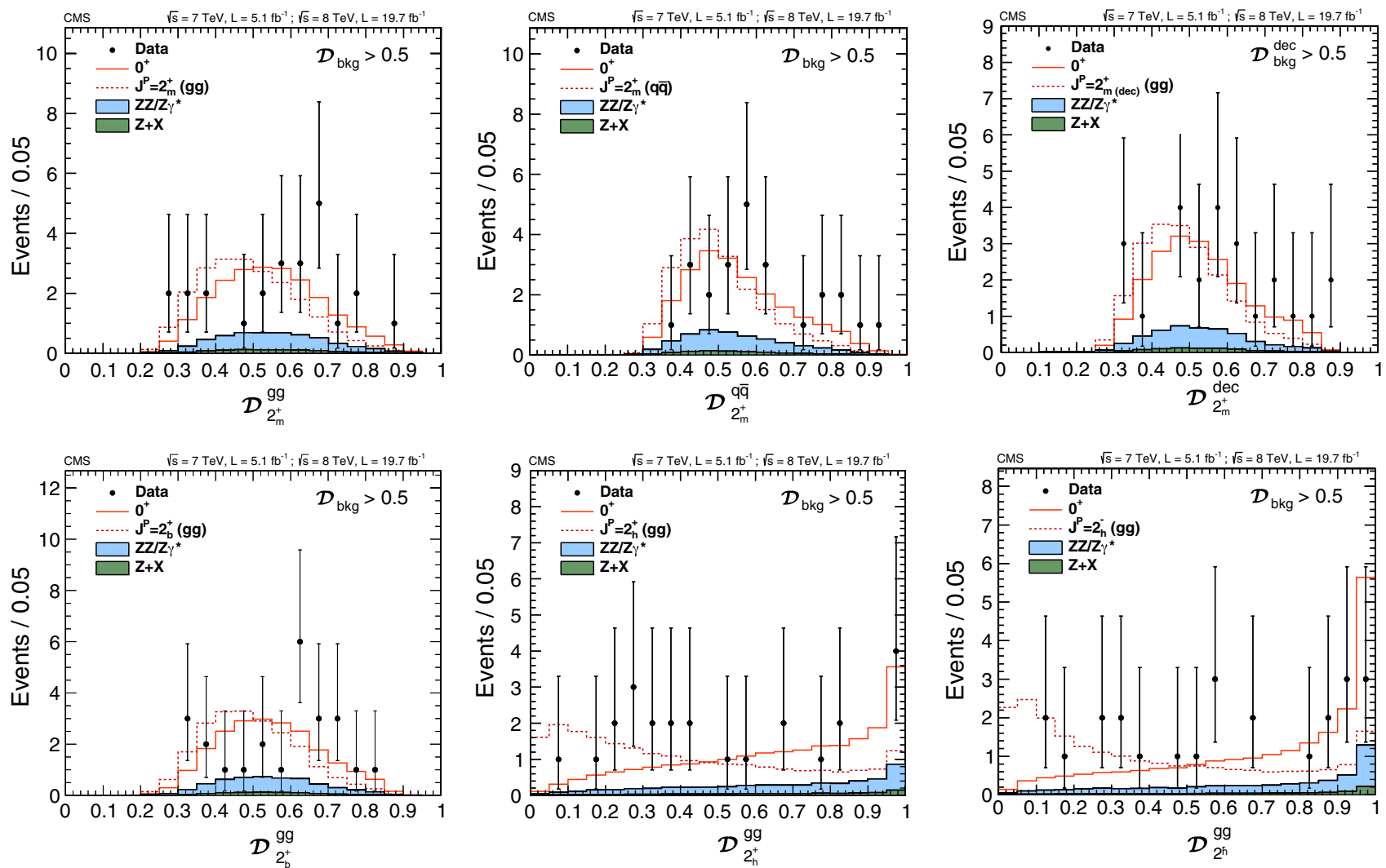

FIG. 25 (color online). Distributions of $\mathscr{D}_{J^{P}}$ with a requirement $\mathscr{D}_{\mathrm{bkg}}^{(\mathrm{dec})}>0.5$. Distributions in data (points with error bars) and expectations for background and signal are shown: six alternative $J^{P}$ hypotheses are shown. $J^{P}=2_{\mathrm{m}}^{+}$for gluon fusion (upper left), $2_{\mathrm{m}}^{+}$ for VBF (upper middle), $2_{\mathrm{m}}^{+}$for the production-independent scenario (upper right), $2_{\mathrm{b}}^{+}(g g)$ (lower left), $2_{\mathrm{h}}^{+}(g g),\left(\right.$ lower middle), $2_{\mathrm{h}}^{-}(g g)$ (lower right). 


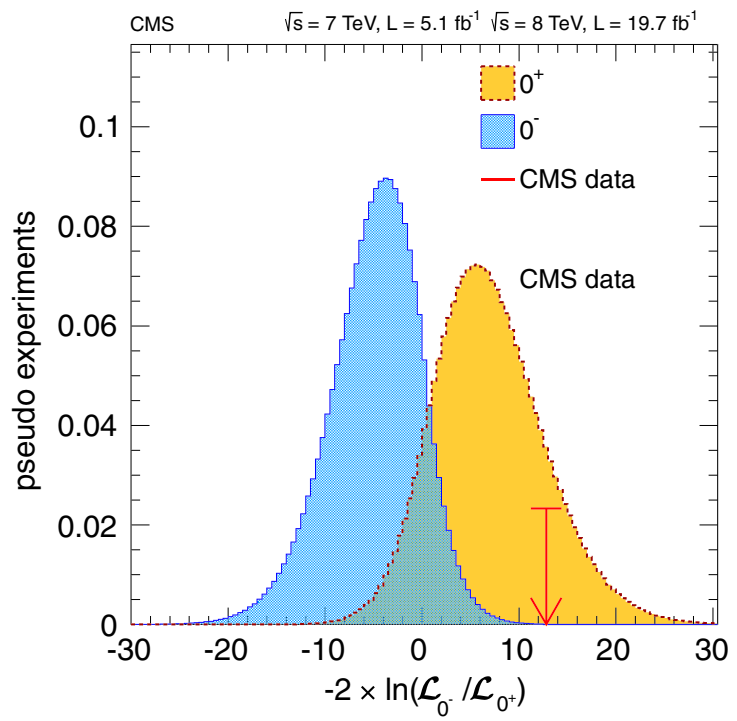

TABLE VIII. List of models used in the analysis of the spin and parity hypotheses corresponding to the pure states of the type noted. The expected separation is quoted for two scenarios, where the signal strength for each hypothesis is predetermined from the fit to data and where events are generated with SM expectations for the signal cross section $(\mu=1)$. The observed separation quotes consistency of the observation with the $0^{+}$model or $J^{P}$ model and corresponds to the scenario where the signal strength is floated in the fit to data. The last column quotes the $\mathrm{CL}_{\mathrm{s}}$ value for the $J^{P}$ model.

\begin{tabular}{|c|c|c|c|c|c|}
\hline $\begin{array}{l}J^{P} \\
\text { model }\end{array}$ & $\begin{array}{c}J^{P} \\
\text { production }\end{array}$ & $\begin{array}{l}\text { Expected } \\
(\mu=1)\end{array}$ & Obs. $0^{+}$ & Obs. $J^{P}$ & $\mathrm{CL}_{\mathrm{s}}$ \\
\hline $0^{-}$ & any & $2.4 \sigma(2.7 \sigma)$ & $-1.0 \sigma$ & $+3.8 \sigma$ & $0.05 \%$ \\
\hline $0_{\mathrm{h}}^{+}$ & any & $1.7 \sigma(1.9 \sigma)$ & $-0.3 \sigma$ & $+2.1 \sigma$ & $4.5 \%$ \\
\hline $1^{-}$ & $q \bar{q} \rightarrow X$ & $2.7 \sigma(2.7 \sigma)$ & $-1.4 \sigma$ & $+4.7 \sigma$ & $0.002 \%$ \\
\hline $1^{-}$ & any & $2.5 \sigma(2.6 \sigma)$ & $-1.8 \sigma$ & $+4.9 \sigma$ & $0.001 \%$ \\
\hline $1^{+}$ & $q \bar{q} \rightarrow X$ & $2.1 \sigma(2.3 \sigma)$ & $-1.5 \sigma$ & $+4.1 \sigma$ & $0.02 \%$ \\
\hline $1^{+}$ & any & $2.0 \sigma(2.1 \sigma)$ & $-2.1 \sigma$ & $+4.8 \sigma$ & $0.004 \%$ \\
\hline $2_{\mathrm{m}}^{+}$ & $g g \rightarrow X$ & $1.9 \sigma(1.8 \sigma)$ & $-1.1 \sigma$ & $+3.0 \sigma$ & $0.9 \%$ \\
\hline $2_{\mathrm{m}}^{+}$ & $q \bar{q} \rightarrow X$ & $1.7 \sigma(1.7 \sigma)$ & $-1.7 \sigma$ & $+3.8 \sigma$ & $0.2 \%$ \\
\hline $2_{\mathrm{m}}^{+}$ & any & $1.5 \sigma(1.5 \sigma)$ & $-1.6 \sigma$ & $+3.4 \sigma$ & $0.7 \%$ \\
\hline $2_{\mathrm{b}}^{+}$ & $g g \rightarrow X$ & $1.6 \sigma(1.8 \sigma)$ & $-1.4 \sigma$ & $+3.4 \sigma$ & $0.5 \%$ \\
\hline $2_{\mathrm{h}}^{+}$ & $g g \rightarrow X$ & $3.8 \sigma(4.0 \sigma)$ & $+1.8 \sigma$ & $+2.0 \sigma$ & $2.3 \%$ \\
\hline $2_{\mathrm{h}}^{-}$ & $g g \rightarrow X$ & $4.2 \sigma(4.5 \sigma)$ & $+1.0 \sigma$ & $+3.2 \sigma$ & $0.09 \%$ \\
\hline
\end{tabular}

For example, even when restricting the coupling ratios to be real, there remains an ambiguity where $\phi_{a 3}=0$ or $\pi$. The interference between the two terms $\left(a_{1}\right.$ and $\left.a_{3}\right)$ is found to have a negligible effect on the discriminant distribution or the overall yield of events. The parameter $f_{a 3}$ is defined as

$$
f_{a 3}=\frac{\left|a_{3}\right|^{2} \sigma_{3}}{\left|a_{1}\right|^{2} \sigma_{1}+\left|a_{2}\right|^{2} \sigma_{2}+\left|a_{3}\right|^{2} \sigma_{3}}
$$

FIG. 26 (color online). (top) Distribution of the test statistic $q=-2 \ln \left(\mathscr{L}_{0^{-}} / \mathscr{L}_{0^{+}}\right)$of the pseudoscalar boson hypothesis tested against the SM Higgs boson hypothesis. Distributions for the SM Higgs boson are represented by the yellow histogram, and those for the alternative $J^{P}$ hypotheses are represented by the blue histogram. The arrow indicates the observed value. (bottom) Average expected and observed distribution of $-2 \Delta \ln L$ as a function of $f_{a 3}$. The horizontal lines at $-2 \Delta \ln \mathscr{L}=1$ and 3.84 represent the $68 \%$ and $95 \%$ C.L.'s, respectively.

95\% or higher C.L. The $0_{\mathrm{h}}^{+}$hypothesis is disfavored, with a $\mathrm{CL}_{s}$ value of $4.5 \%$.

In addition to testing pure $J^{P}$ states against the SM Higgs boson hypothesis, a measurement for a possible mixture of $\mathrm{CP}$-even and CP-odd states or other effects leading to anomalous couplings in the $H \rightarrow Z Z$ decay amplitude in Eq. (6) is performed. The $\mathscr{D}_{0^{-}}$discriminant is designed for the discrimination between the third and the first amplitude contributions in Eq. (6) when the phase $\phi_{a 3}$ between $a_{3}$ and $a_{1}$ couplings is not determined from the data [48].

where $\sigma_{i}$ is the effective cross section $H \rightarrow Z Z \rightarrow 2 e 2 \mu$ corresponding to $a_{i}=1, a_{j \neq i}=0$. The $4 e$ and $4 \mu$ final

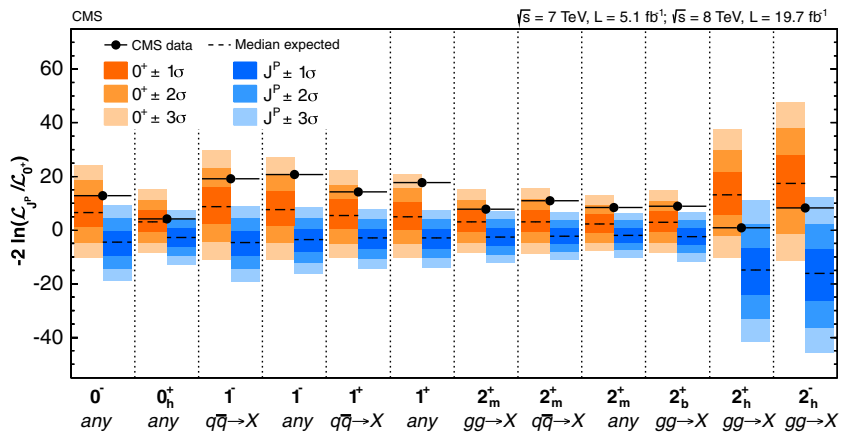

FIG. 27 (color online). Summary of the expected and observed values for the test-statistic $q$ distributions for the twelve alternative hypotheses tested with respect to the SM Higgs boson. The orange (blue) bands represent $1 \sigma, 2 \sigma$, and $3 \sigma$ around the median expected value for the SM Higgs boson hypothesis (alternative hypothesis). The black point represents the observed value. 
states may lead to either constructive or destructive interference of identical leptons, and therefore to slightly different cross-section ratios. When testing the CP-odd contribution, the second term in the amplitude is assumed to be zero $\left(a_{2}=0\right)$. The measured value of $f_{a 3}$ can be used to extract the coupling constants in any parameterization. For example, following Eq. (6), the couplings are

$$
\frac{\left|a_{3}\right|}{\left|a_{1}\right|}=\sqrt{\frac{f_{a 3}}{\left(1-f_{a 3}\right)}} \times \sqrt{\frac{\sigma_{1}}{\sigma_{3}}},
$$

where $\sigma_{1} / \sigma_{3}=6.36$ for a boson with mass $125.6 \mathrm{GeV}$. The $f_{a 3}$ parameter does not define the mixture of parity-even and parity-odd states, because it would also depend on the relative strength of their couplings to vector bosons.

Figure 26 (bottom) shows a likelihood scan of $-2 \ln \mathscr{L}$, where the likelihood for the event $i, \mathscr{L}^{i} \equiv \mathscr{L}_{f_{a 3}}^{i} \propto$ $\left(1-f_{a 3}\right) \mathscr{L}_{2 D}^{i, 0^{+}}+f_{a 3} \mathscr{L}_{2 D}^{i, 0^{-}}$. The normalization due to the acceptance is accounted for in $\mathscr{L}_{2 D}^{J^{P}}$, defined in Eq. (15), and the normalization of the likelihood $\mathscr{L}_{f_{a 3}}^{i}$ depends on $f_{a 3}$. From the likelihood scan as a function of $f_{a 3}$, the fraction of a $\mathrm{CP}$-odd amplitude contribution to the cross section $f_{a 3}=0.00_{-0.00}^{+0.17}$, and a limit $f_{a 3}<0.51$ at the 95\% C.L., are inferred. The limit on $f_{a 3}$ can be converted into a limit on amplitude constants using the convention of Eq. (6): $\left|a_{3} / a_{1}\right|<2.6$ at the $95 \%$ C.L. The statistical coverage of the results obtained in the likelihood scan has also been tested, with the Feldman-Cousins approach [153] yielding a consistent result.

\section{SUMMARY}

The observation and the measurements of the properties of a Higgs boson candidate in the four-lepton decay channel have been presented. The four-lepton invariant mass distributions are presented for $m_{4 \ell}>70 \mathrm{GeV}$ using data samples corresponding to integrated luminosities of $5.1 \mathrm{fb}^{-1}$ at $\sqrt{\mathrm{s}}=$ $7 \mathrm{TeV}$ and $19.7 \mathrm{fb}^{-1}$ at $\sqrt{s}=8 \mathrm{TeV}$. For the measurements, the following experimental observables are employed: the measured four-lepton mass, the mass uncertainty, kinematic discriminants, and information sensitive to the production mechanism, such as associated dijet characteristics and transverse momentum of the four-lepton system.

The observation of the new boson [20,21,31] is confirmed in the $4 \ell$ final state, with a local significance of 6.8 standard deviations above the expected background. Upper limits at the $95 \%$ C.L. exclude the SM-like Higgs boson in the mass ranges 114.5-119.0 GeV and 129.5-832.0 GeV, for an expected exclusion range for the background-only hypothesis of $115-740 \mathrm{GeV}$. The measured mass of the new boson is $125.6 \pm 0.4$ (stat) \pm 0.2 (syst) GeV. The measured width of this resonance is smaller than $3.4 \mathrm{GeV}$ at the 95\% C.L. The production cross section of the new boson times the branching fraction to four leptons is measured to be $0.93_{-0.23}^{+0.26}$ (stat $)_{-0.09}^{+0.13}$ (syst) times that predicted by the standard model. Those associated with fermions and vector bosons are $\mu_{g g H, t \bar{t} H}=0.80_{-0.36}^{+0.46}$ and $\mu_{\mathrm{VBF}, \mathrm{VH}}=1.7_{-2.1}^{+2.2}$, respectively, consistent with the SM expectations.

The spin parity of the boson is studied, and the observation is consistent with the pure scalar hypothesis when compared to several other spin-parity hypotheses. The fraction of a CP-odd contribution to the decay amplitude, expressed through the fraction $f_{a 3}$ of the corresponding decay rate, is $f_{a 3}=0.00_{-0.00}^{+0.17}$, and thus consistent with the expectation for the SM Higgs boson. The hypotheses of a pseudoscalar and all tested spin-1 boson hypotheses are excluded at the $99 \%$ C.L. or higher. All tested spin-2 boson hypotheses are excluded at the 95\% C.L. or higher.

The production and decay properties of the observed new boson in the four-lepton final state are consistent, within their uncertainties, with the expectations for the SM Higgs boson.

\section{ACKNOWLEDGMENTS}

We congratulate our colleagues in the CERN accelerator departments for the excellent performance of the LHC and thank the technical and administrative staffs at CERN and at other CMS institutes for their contributions to the success of the CMS effort. In addition, we gratefully acknowledge the computing centers and personnel of the Worldwide LHC Computing Grid for delivering so effectively the computing infrastructure essential to our analyses. Finally, we acknowledge the enduring support for the construction and operation of the LHC and the CMS detector provided by the following funding agencies: the Austrian Federal Ministry of Science and Research and the Austrian Science Fund; the Belgian Fonds de la Recherche Scientifique and Fonds voor Wetenschappelijk Onderzoek; the Brazilian Funding Agencies (CNPq, CAPES, FAPERJ, and FAPESP); the Bulgarian Ministry of Education and Science; CERN; the Chinese Academy of Sciences, Ministry of Science and Technology, and National Natural Science Foundation of China; the Colombian Funding Agency (COLCIENCIAS); the Croatian Ministry of Science, Education and Sport, and the Croatian Science Foundation; the Research Promotion Foundation, Cyprus; the Ministry of Education and Research, Recurrent Financing Contract No. SF0690030s09 and European Regional Development Fund, Estonia; the Academy of Finland, Finnish Ministry of Education and Culture, and Helsinki Institute of Physics; the Institut National de Physique Nucléaire et de Physique des Particules/CNRS and Commissariat à l'Énergie Atomique et aux Énergies Alternatives/CEA, France; the Bundesministerium für Bildung und Forschung, Deutsche Forschungsgemeinschaft, and Helmholtz-Gemeinschaft Deutscher Forschungszentren, Germany; the General Secretariat for Research and Technology, Greece; the National Scientific Research Foundation and National 
Innovation Office, Hungary; the Department of Atomic Energy and the Department of Science and Technology, India; the Institute for Studies in Theoretical Physics and Mathematics, Iran; the Science Foundation, Ireland; the Istituto Nazionale di Fisica Nucleare, Italy; the Korean Ministry of Education, Science and Technology and the World Class University program of NRF, Republic of Korea; the Lithuanian Academy of Sciences; the Mexican Funding Agencies (CINVESTAV, CONACYT, SEP, and UASLP-FAI); the Ministry of Business, Innovation and Employment, New Zealand; the Pakistan Atomic Energy Commission; the Ministry of Science and Higher Education and the National Science Centre, Poland; the Fundação para a Ciência e a Tecnologia, Portugal; JINR, Dubna, the Ministry of Education and Science of the Russian Federation, the Federal Agency of Atomic Energy of the Russian Federation, Russian Academy of Sciences, and the Russian Foundation for Basic Research; the Ministry of Education, Science and Technological Development of Serbia; the Secretaría de Estado de Investigación, Desarrollo e Innovación and Programa Consolider-Ingenio 2010, Spain; the Swiss Funding Agencies (ETH Board, ETH Zurich, PSI, SNF, UniZH, Canton Zurich, and SER); the National Science Council, Taipei; the Thailand Center of Excellence in Physics, the
Institute for the Promotion of Teaching Science and Technology of Thailand, Special Task Force for Activating Research and the National Science and Technology Development Agency of Thailand; the Scientific and Technical Research Council of Turkey and the Turkish Atomic Energy Authority; the Science and Technology Facilities Council, United Kingdom; the U.S. Department of Energy and the U.S. National Science Foundation. Individuals have received support from the Marie-Curie program and the European Research Council and EPLANET (European Union); the Leventis Foundation; the A.P. Sloan Foundation; the Alexander von Humboldt Foundation; the Belgian Federal Science Policy Office; the Fonds pour la Formation à la Recherche dans l'Industrie et dans l'Agriculture (FRIA-Belgium); the Agentschap voor Innovatie door Wetenschap en Technologie (IWT-Belgium); the Ministry of Education, Youth and Sports (MEYS) of the Czech Republic; the Council of Science and Industrial Research, India; the Compagnia di San Paolo (Torino); the HOMING PLUS programme of Foundation for Polish Science, cofinanced by EU, Regional Development Fund; and the Thalis and Aristeia programmes cofinanced by EU-ESF and the Greek NSRF.
[1] S. L. Glashow, Nucl. Phys. 22, 579 (1961).

[2] S. Weinberg, Phys. Rev. Lett. 19, 1264 (1967).

[3] A. Salam, in Elementary Particle Physics: Relativistic Groups and Analyticity, edited by N. Svartholm (Almqvist \& Wiksell, Stockholm, 1968), p. 367.

[4] D. J. Gross and F. Wilczek, Phys. Rev. Lett. 30, 1343 (1973).

[5] H. D. Politzer, Phys. Rev. Lett. 30, 1346 (1973).

[6] F. Englert and R. Brout, Phys. Rev. Lett. 13, 321 (1964).

[7] P. W. Higgs, Phys. Lett. 12, 132 (1964).

[8] P. W. Higgs, Phys. Rev. Lett. 13, 508 (1964).

[9] G. S. Guralnik, C. R. Hagen, and T. W. B. Kibble, Phys. Rev. Lett. 13, 585 (1964).

[10] P. W. Higgs, Phys. Rev. 145, 1156 (1966).

[11] T. W. B. Kibble, Phys. Rev. 155, 1554 (1967).

[12] Y. Nambu and G. Jona-Lasinio, Phys. Rev. 122, 345 (1961).

[13] M. Gell-Mann and M. Levy, Nuovo Cimento 16, 705 (1960).

[14] J. M. Cornwall, D. N. Levin, and G. Tiktopoulos, Phys. Rev. Lett. 30, 1268 (1973).

[15] J. M. Cornwall, D. N. Levin, and G. Tiktopoulos, Phys. Rev. D 10, 1145 (1974)11972(E) (1975).

[16] C. H. Llewellyn Smith, Phys. Lett. 46B, 233 (1973).

[17] B. W. Lee, C. Quigg, and H. B. Thacker, Phys. Rev. D 16, 1519 (1977).

[18] ALEPH, CDF, D0, DELPHI, L3, OPAL, and SLD Collaborations, the LEP Electroweak Working Group, the Tevatron Electroweak Working Group, and the SLD Electroweak and Heavy Flavour Groups, CERN Report No. PH-EP-2010-095, 2010; At this time, the most up-to-date Higgs boson mass constraints come from lepewwg.web.cern.ch/LEPEWWG/plots/winter2012, arXiv:1012.2367.

[19] ATLAS Collaboration, Phys. Lett. B 716, 1 (2012).

[20] CMS Collaboration, Phys. Lett. B 716, 30 (2012).

[21] CMS Collaboration, J. High Energy Phys. 06 (2013) 081.

[22] ALEPH, DELPHI, L3, and OPAL Collaborations, and the LEP Working Group for Higgs Boson Searches, Phys. Lett. B 565, 61 (2003).

[23] T. Aaltonen et al. (CDF and D0 Collaborations), Phys. Rev. Lett. 109, 071804 (2012).

[24] CDF and D0 Collaborations, Phys. Rev. D 88, 052014 (2013).

[25] ATLAS Collaboration, Phys. Lett. B 710, 383 (2012).

[26] ATLAS Collaboration, Phys. Lett. B 710, 49 (2012).

[27] ATLAS Collaboration, Phys. Rev. D 86, 032003 (2012).

[28] CMS Collaboration, Phys. Rev. Lett. 108, 111804 (2012).

[29] CMS Collaboration, J. High Energy Phys. 03 (2012) 081.

[30] CMS Collaboration, Phys. Lett. B 710, 26 (2012).

[31] CMS Collaboration, Phys. Rev. Lett. 110, 081803 (2013).

[32] ATLAS Collaboration, Phys. Lett. B 726, 88 (2013).

[33] ATLAS Collaboration, Phys. Lett. B 726, 120 (2013).

[34] CMS Collaboration, JINST 3, S08004 (2008). 
[35] CMS Collaboration, JINST 5, T03008 (2010).

[36] CMS Collaboration, JINST 5, T03009 (2010).

[37] CMS Collaboration, JINST 8, P09009 (2013).

[38] S. Frixione, P. Nason, and C. Oleari, J. High Energy Phys. 11 (2007) 070.

[39] E. Bagnaschi, G. Degrassi, P. Slavich, and A. Vicini, J. High Energy Phys. 02 (2012) 088.

[40] P. Nason and C. Oleari, J. High Energy Phys. 02 (2010) 037.

[41] Y. Gao, A. V. Gritsan, Z. Guo, K. Melnikov, M. Schulze, and N. V. Tran, Phys. Rev. D 81, 075022 (2010).

[42] S. Bolognesi, Y. Gao, A. V. Gritsan, K. Melnikov, M. Schulze, N. V. Tran, and A. Whitbeck, Phys. Rev. D 86, 095031 (2012).

[43] I. Anderson, S. Bolognesi, F. Caola, Y. Gao, A. V. Gritsan, C. B. Martin, K. Melnikov, M. Schulze, N. V. Tran, A. Whitbeck, and Y. Zhou, Phys. Rev. D 89, 035007 (2014).

[44] T. Sjöstrand, S. Mrenna, and P. Z. Skands, J. High Energy Phys. 05 (2006) 026.

[45] G. Passarino, C. Sturm, and S. Uccirati, Nucl. Phys. B834, 77 (2010).

[46] S. Goria, G. Passarino, and D. Rosco, Nucl. Phys. B864, 530 (2012).

[47] N. Kauer and G. Passarino, J. High Energy Phys. 08 (2012) 116.

[48] LHC Higgs Cross Section Working Group, CERN Report No. CERN-2013-004, 2013.

[49] G. Passarino, J. High Energy Phys. 08 (2012) 146.

[50] N. Kauer, Proc. Sci., RADCOR2011 (2011) 027 [arXiv:1201.1667].

[51] D. de Florian, G. Ferrera, M. Grazzini, and D. Tommasini, J. High Energy Phys. 06 (2012) 132.

[52] C. Anastasiou, R. Boughezal, and F. Petriello, J. High Energy Phys. 04 (2009) 003.

[53] D. de Florian and M. Grazzini, Phys. Lett. B 674, 291 (2009).

[54] J. Baglio and A. Djouadi, J. High Energy Phys. 03 (2011) 055.

[55] LHC Higgs Cross Section Working Group, CERN Report No. CERN-2011-002, 2011.

[56] A. Djouadi, M. Spira, and P. M. Zerwas, Phys. Lett. B 264, 440 (1991).

[57] S. Dawson, Nucl. Phys. B359, 283 (1991).

[58] M. Spira, A. Djouadi, D. Graudenz, and R. M. Zerwas, Nucl. Phys. B453, 17 (1995).

[59] R. V. Harlander and W. B. Kilgore, Phys. Rev. Lett. 88, 201801 (2002).

[60] C. Anastasiou and K. Melnikov, Nucl. Phys. B646, 220 (2002).

[61] V. Ravindran, J. Smith, and W. L. van Neerven, Nucl. Phys. B665, 325 (2003).

[62] S. Catani, D. de Florian, M. Grazzini, and P. Nason, J. High Energy Phys. 07 (2003) 028.

[63] S. Actis, G. Passarino, C. Sturm, and S. Uccirati, Phys. Lett. B 670, 12 (2008).

[64] M. Ciccolini, A. Denner, and S. Dittmaier, Phys. Rev. Lett. 99, 161803 (2007).

[65] M. Ciccolini, A. Denner, and S. Dittmaier, Phys. Rev. D 77, 013002 (2008).

[66] T. Figy, D. Zeppenfeld, and C. Oleari, Phys. Rev. D 68, 073005 (2003).

[67] K. Arnold et al., Comput. Phys. Commun. 180, 1661 (2009).
[68] P. Bolzoni, F. Maltoni, S.-O. Moch, and M. Zaro, Phys. Rev. Lett. 105, 011801 (2010).

[69] T. Melia, P. Nason, R. Rontsch, and G. Zanderighi, J. High Energy Phys. 11 (2011) 078.

[70] J. Alwall, P. Demin, S. de Visscher, R. Frederix, M. Herquet, F. Maltoni, T. Plehn, D. L. Rainwater, and T. Stelzer, J. High Energy Phys. 09 (2007) 028.

[71] T. Binoth, N. Kauer, and P. Mertsch, arXiv:0807.0024.

[72] H.-L. Lai, J. Huston, Z. Li, P. Nadolsky, J. Pumplin, D. Stump, and C.-P. Yuan, Phys. Rev. D 82, 054021 (2010).

[73] H.-L. Lai, M. Guzzi, J. Huston, Z. Li, P. M. Nadolsky, J. Pumplin, and C.-P. Yuan, Phys. Rev. D 82, 074024 (2010).

[74] CMS Collaboration, J. High Energy Phys. 09 (2011) 109.

[75] S. Agostinelli et al. (GEANT4), Nucl. Instrum. Methods Phys. Res., Sect. A 506, 250 (2003).

[76] J. Allison et al., IEEE Trans. Nucl. Sci. 53, 270 (2006).

[77] LHC Higgs Cross Section Working Group, CERN Report No. CERN-2012-002, 2012.

[78] CMS Collaboration, CMS Physics Analysis Summary No. CMS-PAS-PFT-09-001, 2009, http://cdsweb.cern.ch/ record/1194487.

[79] CMS Collaboration, CMS Physics Analysis Summary No. CMS-PAS-PFT-10-001, 2010, http://cdsweb.cern.ch/ record/1247373.

[80] CMS Collaboration, CMS Physics Analysis Summary No. CMS-PAS-PFT-10-002, 2010, http://cdsweb.cern.ch/ record/1279341.

[81] CMS Collaboration, CMS Physics Analysis Summary No. CMS-PAS-PFT-10-003, 2010, http://cdsweb.cern.ch/ record/1279347.

[82] S. Baffioni, C. Charlot, F. Ferri, D. Futyan, P. Meridiani, I. Puljak, C. Rovelli, R. Salerno, and Y. Sirois, Eur. Phys. J. C 49, 1099 (2007).

[83] CMS Collaboration, CMS Physics Analysis Summary No. CMS-PAS-EGM-10-004, 2010, http://cdsweb.cern .ch/record/1299116.

[84] CMS Collaboration, CMS Detector Performance Summary No. CMS-DP-2011-003, 2011, http://cdsweb.cern .ch/record/1360227.

[85] CMS Collaboration, CMS Detector Performance Summary No. CMS-DP-2013-003, 2013, http://cdsweb.cern .ch/record/1523273.

[86] W. Adam, R. Frühwirth, A. Strandlie, and T. Todorov, J. Phys. G 31, N9 (2005).

[87] CMS Collaboration, No. CMS-DP-2012-015, 2012, http:// cdsweb.cern.ch/record/1478128.

[88] H. Voss, A. Höcker, J. Stelzer, and F. Tegenfeldt, Proc. Sci., ACAT2007 (2007) 040 [arXiv:physics/0703039].

[89] CMS Collaboration, JINST 5, T03010 (2010).

[90] M. Oreglia, Ph.D. thesis, Stanford University, 1980.

[91] CMS Collaboration, JINST 7, P10002 (2012).

[92] CMS Collaboration, arXiv:1403.2286 [JINST (in preparation)].

[93] M. Cacciari and G. P. Salam, Phys. Lett. B 659, 119 (2008).

[94] M. Cacciari, G. P. Salam, and G. Soyez, J. High Energy Phys. 04 (2008) 005.

[95] M. Cacciari, G. P. Salam, and G. Soyez, Eur. Phys. J. C 72, 1896 (2012).

[96] S. Catani, Y. L. Dokshitzer, M. Seymour, and B. Webber, Nucl. Phys. B406, 187 (1993). 
[97] S. D. Ellis and D. E. Soper, Phys. Rev. D 48, 3160 (1993).

[98] S. Baffioni, C. Charlot, F. Ferri, N. Godinovic, P. Meridiani, I. Puljak, R. Salerno, and Y. Sirois, J. Phys. G 34, N23 (2007).

[99] CMS Collaboration, J. High Energy Phys. 10 (2011) 132.

[100] M. Cacciari, G. P. Salam, and G. Soyez, J. High Energy Phys. 04 (2008) 063.

[101] M. Cacciari and G. P. Salam, Phys. Lett. B 641, 57 (2006).

[102] CMS Collaboration, JINST 6, P11002 (2011).

[103] CMS Collaboration, CMS Physics Analysis Summary No. CMS-PAS-JME-13-005, 2013, http://cdsweb.cern .ch/record/1581583.

[104] J. M. Campbell and R. K. Ellis, Nucl. Phys. B, Proc. Suppl. 205-206, 10 (2010).

[105] J. M. Campbell and R. K. Ellis, Phys. Rev. D 60, 113006 (1999).

[106] J. M. Campbell, R. K. Ellis, and C. Williams, J. High Energy Phys. 07 (2011) 018.

[107] ATLAS Collaboration, New J. Phys. 15, 033038 (2013).

[108] L. Landau, J. Phys. (USSR) 8, 201 (1944).

[109] S. Y. Choi, D. J. Miller, M. M. Mühlleitner, and P. M. Zerwas, Phys. Lett. B 553, 61 (2003).

[110] A. Soni and R. M. Xu, Phys. Rev. D 48, 5259 (1993).

[111] V. D. Barger, K.-m. Cheung, A. Djouadi, B. A. Kniehl, and P. M. Zerwas, Phys. Rev. D 49, 79 (1994).

[112] B. C. Allanach, K. Odagiri, M. J. Palmer, M. A. Parker, A. Sabetfakhri, and B. R. Webber, J. High Energy Phys. 12 (2002) 039.

[113] C. P. Buszello, I. Fleck, P. Marquard, and J. J. van der Bij, Eur. Phys. J. C 32, 209 (2004).

[114] R. M. Godbole, D. J. Miller, and M. M. Mühlleitner, J. High Energy Phys. 12 (2007) 031.

[115] W.-Y. Keung, I. Low, and J. Shu, Phys. Rev. Lett. 101, 091802 (2008).

[116] O. Antipin and A. Soni, J. High Energy Phys. 10 (2008) 018.

[117] K. Hagiwara, Q. Li, and K. Mawatari, J. High Energy Phys. 07 (2009) 101.

[118] A. De Rujula, J. Lykken, M. Pierini, C. Rogan, and M. Spiropulu, Phys. Rev. D 82, 013003 (2010).

[119] J. S. Gainer, K. Kumar, I. Low, and R. Vega-Morales, J. High Energy Phys. 11 (2011) 027.

[120] Y. Chen, N. Tran, and R. Vega-Morales, J. High Energy Phys. 01 (2013) 182.

[121] P. Avery, D. Bourilkov, M. Chen, T. Cheng, A. Drozdetskiy, J.S. Gainer, A. Korytov, K. T. Matchev, P. Milenovic, G. Mitselmakher, M. Park, A. Rinkevicius, and M. Snowball, Phys. Rev. D 87, 055006 (2013).

[122] P. Artoisenet, P. de Aquino, F. Demartin, R. Frederix, S. Frixione, F. Maltoni, M. K. Mandal, P. Mathews, K. Mawatari, V. Ravindran, S. Seth, P. Torrielli, and M. Zaro, J. High Energy Phys. 11 (2013) 043.

[123] N. Cabibbo and A. Maksymowicz, Phys. Rev. 168, 1926 (1968).

[124] L. Landau, Dokl. Akad. Nawk. Ser. Fiz. (USSR) 60, 207 (1948).
[125] C.-N. Yang, Phys. Rev. 77, 242 (1950).

[126] L. Randall and R. Sundrum, Phys. Rev. Lett. 83, 4690 (1999).

[127] L. Randall and R. Sundrum, Phys. Rev. Lett. 83, 3370 (1999).

[128] K. Agashe, H. Davoudiasl, G. Perez, and A. Soni, Phys. Rev. D 76, 036006 (2007).

[129] N. D. Christensen and C. Duhr, Comput. Phys. Commun. 180, 1614 (2009).

[130] R. M. Neal, Bayesian Learning for Neural Networks, Lecture Notes in Statistics Vol. 118 (Springer-Verlag, Berlin, 1996).

[131] J. Lampinen and A. Vehtari, Neural Netw. 14, 257 (2001).

[132] L. Breiman, J. H. Friedman, R. A. Olshen, and C. J. Stone, Classification and Regression Trees (Wadsworth, Monterey, CA, 1984).

[133] J. H. Friedman, Computational Statistics and Data Analysis 38, 367 (2002).

[134] R. D. Cousins, Am. J. Phys. 63, 398 (1995).

[135] M. Pivk and F. R. Le Diberder, Nucl. Instrum. Methods Phys. Res., Sect. A 555, 356 (2005).

[136] ATLAS and CMS Collaborations, LHC Higgs Combination Group, ATLAS Report No. ATL-PHYS-PUB 201111/CMS Note No. 2011/005, 2011, http://cds.cern.ch/ record/1379837.

[137] G. Bozzi, S. Catani, D. de Florian, and M. Grazzini, Nucl. Phys. B737, 73 (2006).

[138] D. de Florian, G. Ferrera, M. Grazzini, and D. Tommasini, J. High Energy Phys. 11 (2011) 064.

[139] G. Bozzia, S. Catani, D. de Florian, and M. Grazzini, Phys. Lett. B 564, 65 (2003).

[140] S. Alekhin et al. (PDF4LHC Working Group), arXiv:1101.0536.

[141] M. Botje et al. (PDF4LHC Working Group), arXiv:1101.0538.

[142] A. D. Martin, W. J. Stirling, R. S. Thorne, and G. Watt, Eur. Phys. J. C 63, 189 (2009).

[143] R. D. Ball, V. Bertone, F. Cerutti, L. D. Debbio, S. Forte, A. Guffanti, J. I. Latorre, J. Rojo, and M. Ubiali (NNPDF), Nucl. Phys. B849, 296 (2011).

[144] CMS Collaboration, CMS Report No. CMS-PAS-EWK11-001, 2011, http://cds.cern.ch/record/1376102.

[145] CMS Collaboration, CMS Physics Analysis Summary No. CMS-PAS-LUM-13-001, 2013, http://cds.cern.ch/ record/1598864.

[146] A. Denner, S. Heinemeyer, I. Puljak, D. Rebuzzi, and M. Spira, Eur. Phys. J. C 71, 1753 (2011).

[147] T. Junk, Nucl. Instrum. Methods Phys. Res., Sect. A 434, 435 (1999).

[148] A. L. Read, J. Phys. G 28, 2693 (2002).

[149] J. Beringer et al. (Particle Data Group), Phys. Rev. D 86, 010001 (2012).

[150] G. Cowan, K. Cranmer, E. Gross, and O. Vitells, Eur. Phys. J. C 71, 1 (2011).

[151] E. Gross and O. Vitells, Eur. Phys. J. C 70, 525 (2010).

[152] CMS Collaboration, J. High Energy Phys. 12 (2012) 034.

[153] G. J. Feldman and R. D. Cousins, Phys. Rev. D 57, 3873 (1998). 
S. Chatrchyan, ${ }^{1}$ V. Khachatryan, ${ }^{1}$ A. M. Sirunyan, ${ }^{1}$ A. Tumasyan, ${ }^{1}$ W. Adam, ${ }^{2}$ T. Bergauer, ${ }^{2}$ M. Dragicevic, ${ }^{2}$ J. Erö, ${ }^{2}$ C. Fabjan, ${ }^{2, b}$ M. Friedl, ${ }^{2}$ R. Frühwirth,,${ }^{2, b}$ V. M. Ghete, ${ }^{2}$ C. Hartl, ${ }^{2}$ N. Hörmann, ${ }^{2}$ J. Hrubec, ${ }^{2}$ M. Jeitler, ${ }^{2, b}$ W. Kiesenhofer, ${ }^{2}$ V. Knünz, ${ }^{2}$ M. Krammer, ${ }^{2, b}$ I. Krätschmer, ${ }^{2}$ D. Liko, ${ }^{2}$ I. Mikulec, ${ }^{2}$ D. Rabady, ${ }^{2, c}$ B. Rahbaran, ${ }^{2}$ H. Rohringer, ${ }^{2}$ R. Schöfbeck, ${ }^{2}$ J. Strauss, ${ }^{2}$ A. Taurok, ${ }^{2}$ W. Treberer-Treberspurg, ${ }^{2}$ W. Waltenberger, ${ }^{2}$ C.-E. Wulz, ${ }^{2, b}$ V. Mossolov, ${ }^{3}$ N. Shumeiko, ${ }^{3}$ J. Suarez Gonzalez, ${ }^{3}$ S. Alderweireldt, ${ }^{4}$ M. Bansal, ${ }^{4}$ S. Bansal, ${ }^{4}$ T. Cornelis, ${ }^{4}$ E. A. De Wolf, ${ }^{4}$ X. Janssen, ${ }^{4}$ A. Knutsson, ${ }^{4}$ S. Luyckx, ${ }^{4}$ L. Mucibello, ${ }^{4}$ S. Ochesanu, ${ }^{4}$ B. Roland, ${ }^{4}$ R. Rougny, ${ }^{4}$ H. Van Haevermaet, ${ }^{4}$ P. Van Mechelen, ${ }^{4}$ N. Van Remortel, ${ }^{4}$ A. Van Spilbeeck, ${ }^{4}$ F. Blekman, ${ }^{5}$ S. Blyweert, ${ }^{5}$ J. D'Hondt, ${ }^{5}$ N. Heracleous, ${ }^{5}$ A. Kalogeropoulos, ${ }^{5}$

J. Keaveney, ${ }^{5}$ T. J. Kim, ${ }^{5}$ S. Lowette, ${ }^{5}$ M. Maes, ${ }^{5}$ A. Olbrechts, ${ }^{5}$ D. Strom, ${ }^{5}$ S. Tavernier, ${ }^{5}$ W. Van Doninck, ${ }^{5}$ P. Van Mulders, ${ }^{5}$ G. P. Van Onsem, ${ }^{5}$ I. Villella,${ }^{5}$ C. Caillol, ${ }^{6}$ B. Clerbaux, ${ }^{6}$ G. De Lentdecker, ${ }^{6}$ L. Favart,${ }^{6}$ A. P. R. Gay, ${ }^{6}$ A. Léonard, ${ }^{6}$ P. E. Marage, ${ }^{6}$ A. Mohammadi, ${ }^{6}$ L. Perniè, ${ }^{6}$ T. Reis,${ }^{6}$ T. Seva, ${ }^{6}$ L. Thomas, ${ }^{6}$ C. Vander Velde, ${ }^{6}$ P. Vanlaer, ${ }^{6}$ J. Wang, ${ }^{6}$ V. Adler, ${ }^{7}$ K. Beernaert, ${ }^{7}$ L. Benucci, ${ }^{7}$ A. Cimmino, ${ }^{7}$ S. Costantini, ${ }^{7}$ S. Dildick, ${ }^{7}$ G. Garcia, ${ }^{7}$ B. Klein, ${ }^{7}$ J. Lellouch, ${ }^{7}$ J. Mccartin, ${ }^{7}$ A. A. Ocampo Rios, ${ }^{7}$ D. Ryckbosch, ${ }^{7}$ S. Salva Diblen, ${ }^{7}$ M. Sigamani, ${ }^{7}$ N. Strobbe, ${ }^{7}$ F. Thyssen, ${ }^{7}$ M. Tytgat, ${ }^{7}$ S. Walsh, ${ }^{7}$ E. Yazgan, ${ }^{7}$ N. Zaganidis, ${ }^{7}$ S. Basegmez,${ }^{8}$ C. Beluffi, ${ }^{8, d}$ G. Bruno, ${ }^{8}$ R. Castello, ${ }^{8}$ A. Caudron, ${ }^{8}$ L. Ceard ${ }^{8}$ G. G. Da Silveira, ${ }^{8}$ C. Delaere, ${ }^{8}$ T. du Pree, ${ }^{8}$ D. Favart, ${ }^{8}$ L. Forthomme, ${ }^{8}$ A. Giammanco, ${ }^{8, e}$ J. Hollar, ${ }^{8}$ P. Jez, ${ }^{8}$ M. Komm, ${ }^{8}$ V. Lemaitre, ${ }^{8}$ J. Liao, ${ }^{8}$ O. Militaru, ${ }^{8}$ C. Nuttens, ${ }^{8}$ D. Pagano, ${ }^{8}$ A. Pin, ${ }^{8}$ K. Piotrzkowski, ${ }^{8}$ A. Popov, ${ }^{8, f}$

L. Quertenmont, ${ }^{8}$ M. Selvaggi, ${ }^{8}$ M. Vidal Marono, ${ }^{8}$ J. M. Vizan Garcia, ${ }^{8}$ N. Beliy, ${ }^{9}$ T. Caebergs, ${ }^{9}$ E. Daubie, ${ }^{9}$ G. H. Hammad, ${ }^{9}$ G. A. Alves, ${ }^{10}$ M. Correa Martins Junior, ${ }^{10}$ T. Martins, ${ }^{10}$ M. E. Pol, ${ }^{10}$ M. H. G. Souza, ${ }^{10}$ W. L. Aldá Júnior, ${ }^{11}$ W. Carvalho, ${ }^{11}$ J. Chinellato, ${ }^{11, g}$ A. Custódio, ${ }^{11}$ E. M. Da Costa, ${ }^{11}$ D. De Jesus Damiao, ${ }^{11}$ C. De Oliveira Martins, ${ }^{11}$ S. Fonseca De Souza, ${ }^{11}$ H. Malbouisson, ${ }^{11}$ M. Malek, ${ }^{11}$ D. Matos Figueiredo, ${ }^{11}$ L. Mundim, ${ }^{11}$ H. Nogima, ${ }^{11}$ W. L. Prado Da Silva, ${ }^{11}$ J. Santaolalla, ${ }^{11}$ A. Santoro, ${ }^{11}$ A. Sznajder, ${ }^{11}$ E. J. Tonelli Manganote, ${ }^{11, g}$ A. Vilela Pereira, ${ }^{11}$ C. A. Bernardes, ${ }^{12 b}$ F. A. Dias, ${ }^{12 a, h}$ T. R. Fernandez Perez Tomei, ${ }^{12 a}$ E. M. Gregores, ${ }^{12 b}$ C. Lagana, ${ }^{12 a}$ P. G. Mercadante, ${ }^{12 b}$ S. F. Novaes, ${ }^{12 a}$ S. S. Padula, ${ }^{12 a}$ V. Genchev,${ }^{13, c}$ P. Iaydjiev, ${ }^{13, c}$ A. Marinov, ${ }^{13}$ S. Piperov, ${ }^{13}$ M. Rodozov, ${ }^{13}$ G. Sultanov, ${ }^{13}$ M. Vutova, ${ }^{13}$ A. Dimitrov, ${ }^{14}$ I. Glushkov, ${ }^{14}$ R. Hadjiiska, ${ }^{14}$ V. Kozhuharov, ${ }^{14}$ L. Litov, ${ }^{14}$ B. Pavlov, ${ }^{14}$ P. Petkov, ${ }^{14}$ J. G. Bian, ${ }^{15}$ G. M. Chen, ${ }^{15}$ H. S. Chen,${ }^{15}$ M. Chen, ${ }^{15}$ R. Du, ${ }^{15}$ C. H. Jiang, ${ }^{15}$ D. Liang, ${ }^{15}$ S. Liang, ${ }^{15}$ X. Meng, ${ }^{15}$ R. Plestina, ${ }^{15, \mathrm{i}} \mathrm{J}$. Tao, ${ }^{15} \mathrm{X}$. Wang, ${ }^{15} \mathrm{Z}$. Wang, ${ }^{15}$ C. Asawatangtrakuldee, ${ }^{16} \mathrm{Y}$. Ban, ${ }^{16} \mathrm{Y} . \mathrm{Guo},{ }^{16} \mathrm{Q} . \mathrm{Li},{ }^{16} \mathrm{~W} . \mathrm{Li},{ }^{16}$ S. Liu, ${ }^{16}$ Y. Mao, ${ }^{16}$ S. J. Qian, ${ }^{16}$ D. Wang, ${ }^{16}$ L. Zhang, ${ }^{16}$ W. Zou, ${ }^{16}$ C. Avila, ${ }^{17}$ C. A. Carrillo Montoya, ${ }^{17}$

L. F. Chaparro Sierra, ${ }^{17}$ C. Florez,${ }^{17}$ J. P. Gomez, ${ }^{17}$ B. Gomez Moreno, ${ }^{17}$ J. C. Sanabria, ${ }^{17}$ N. Godinovic, ${ }^{18}$ D. Lelas, ${ }^{18}$ D. Polic, ${ }^{18}$ I. Puljak,${ }^{18}$ Z. Antunovic, ${ }^{19}$ M. Kovac, ${ }^{19}$ V. Brigljevic, ${ }^{20}$ K. Kadija ${ }^{20}$ J. Luetic, ${ }^{20}$ D. Mekterovic,${ }^{20}$ S. Morovic,${ }^{20}$ L. Tikvica, ${ }^{20}$ A. Attikis, ${ }^{21}$ G. Mavromanolakis, ${ }^{21}$ J. Mousa, ${ }^{21}$ C. Nicolaou, ${ }^{21}$ F. Ptochos,${ }^{21}$ P. A. Razis,${ }^{21}$ M. Finger, ${ }^{22}$ M. Finger Jr., ${ }^{22}$ A. A. Abdelalim, ${ }^{23, j}$ Y. Assran, ${ }^{23, k}$ S. Elgammal, ${ }^{23, j}$ A. Ellithi Kamel, ${ }^{23,1}$ M. A. Mahmoud, ${ }^{23, m}$ A. Radi, ${ }^{23, n, o}$ M. Kadastik,${ }^{24}$ M. Müntel, ${ }^{24}$ M. Murumaa, ${ }^{24}$ M. Raidal,${ }^{24}$ L. Rebane, ${ }^{24}$ A. Tiko, ${ }^{24}$ P. Eerola, ${ }^{25}$ G. Fedi, ${ }^{25}$ M. Voutilainen, ${ }^{25}$ J. Härkönen, ${ }^{26}$ V. Karimäki, ${ }^{26}$ R. Kinnunen, ${ }^{26}$ M. J. Kortelainen, ${ }^{26}$ T. Lampén ${ }^{26}$ K. Lassila-Perini, ${ }^{26}$ S. Lehti, ${ }^{26}$ T. Lindén, ${ }^{26}$ P. Luukka, ${ }^{26}$ T. Mäenpää, ${ }^{26}$ T. Peltola, ${ }^{26}$ E. Tuominen, ${ }^{26}$ J. Tuominiemi,${ }^{26}$ E. Tuovinen, ${ }^{26}$ L. Wendland, ${ }^{26}$ T. Tuuva, ${ }^{27}$ M. Besancon, ${ }^{28}$ F. Couderc ${ }^{28}$ M. Dejardin, ${ }^{28}$ D. Denegri, ${ }^{28}$ B. Fabbro, ${ }^{28}$ J. L. Faure, ${ }^{28}$ F. Ferri, ${ }^{28}$ S. Ganjour, ${ }^{28}$ A. Givernaud ${ }^{28}$ P. Gras, ${ }^{28}$ G. Hamel de Monchenault, ${ }^{28}$ P. Jarry, ${ }^{28}$ E. Locci,${ }^{28}$ J. Malcles, ${ }^{28}$ A. Nayak, ${ }^{28}$ J. Rander, ${ }^{28}$ A. Rosowsky, ${ }^{28}$ M. Titov, ${ }^{28}$ S. Baffioni, ${ }^{29}$ F. Beaudette, ${ }^{29}$ P. Busson, ${ }^{29}$ C. Charlot,${ }^{29}$ N. Daci, ${ }^{29}$ T. Dahms, ${ }^{29}$ M. Dalchenko, ${ }^{29}$ L. Dobrzynski, ${ }^{29}$ A. Florent, ${ }^{29}$ R. Granier de Cassagnac, ${ }^{29}$ P. Miné, ${ }^{29}$ C. Mironov, ${ }^{29}$ I. N. Naranjo, ${ }^{29}$ M. Nguyen, ${ }^{29}$ C. Ochando, ${ }^{29}$ P. Paganini, ${ }^{29}$ D. Sabes,${ }^{29}$ R. Salerno, ${ }^{29}$ Y. Sirois,${ }^{29}$ C. Veelken, ${ }^{29}$ Y. Yilmaz, ${ }^{29}$ A. Zabi, ${ }^{29}$ J.-L. Agram,,${ }^{30, p}$ J. Andrea ${ }^{30}$ D. Bloch, ${ }^{30}$ J.-M. Brom, ${ }^{30}$ E. C. Chabert ${ }^{30}$ C. Collard, ${ }^{30}$ E. Conte, ${ }^{30, p}$ F. Drouhin, ${ }^{30, p}$ J.-C. Fontaine, ${ }^{30, p}$ D. Gelé, ${ }^{30}$ U. Goerlach, ${ }^{30}$ C. Goetzmann, ${ }^{30}$ P. Juillot, ${ }^{30}$ A.-C. Le Bihan, ${ }^{30}$ P. Van Hove, ${ }^{30}$ S. Gadrat,${ }^{31}$ S. Beauceron, ${ }^{32}$ N. Beaupere, ${ }^{32}$ G. Boudoul, ${ }^{32}$ S. Brochet, ${ }^{32}$ J. Chasserat, ${ }^{32}$ R. Chierici, ${ }^{32}$ D. Contardo, ${ }^{32}$ P. Depasse, ${ }^{32}$ H. El Mamouni, ${ }^{32}$ J. Fan, ${ }^{32}$ J. Fay, ${ }^{32}$ S. Gascon, ${ }^{32}$ M. Gouzevitch, ${ }^{32}$ B. Ille, ${ }^{32}$ T. Kurca, ${ }^{32}$ M. Lethuillier, ${ }^{32}$ L. Mirabito, ${ }^{32}$ S. Perries, ${ }^{32}$

J. D. Ruiz Alvarez, ${ }^{32}$ L. Sgandurra, ${ }^{32}$ V. Sordini, ${ }^{32}$ M. Vander Donckt, ${ }^{32}$ P. Verdier, ${ }^{32}$ S. Viret, ${ }^{32}$ H. Xiao, ${ }^{32}$

Z. Tsamalaidze, ${ }^{33, q}$ C. Autermann, ${ }^{34}$ S. Beranek,${ }^{34}$ M. Bontenackels, ${ }^{34}$ B. Calpas, ${ }^{34}$ M. Edelhoff,,${ }^{34}$ L. Feld, ${ }^{34}$ O. Hindrichs, ${ }^{34}$ K. Klein, ${ }^{34}$ A. Ostapchuk, ${ }^{34}$ A. Perieanu,${ }^{34}$ F. Raupach, ${ }^{34}$ J. Sammet, ${ }^{34}$ S. Schael, ${ }^{34}$ D. Sprenger, ${ }^{34}$ H. Weber, ${ }^{34}$ B. Wittmer,${ }^{34}$ V. Zhukov, ${ }^{34, f}$ M. Ata, ${ }^{35}$ J. Caudron,${ }^{35}$ E. Dietz-Laursonn, ${ }^{35}$ D. Duchardt,${ }^{35}$ M. Erdmann, ${ }^{35}$ R. Fischer, ${ }^{35}$ A. Güth, ${ }^{35}$ T. Hebbeker, ${ }^{35}$ C. Heidemann, ${ }^{35}$ K. Hoepfner ${ }^{35}$ D. Klingebiel, ${ }^{35}$ S. Knutzen,${ }^{35}$ P. Kreuzer, ${ }^{35}$ M. Merschmeyer, ${ }^{35}$ A. Meyer,${ }^{35}$ M. Olschewski, ${ }^{35}$ K. Padeken, ${ }^{35}$ P. Papacz,${ }^{35}$ H. Reithler, ${ }^{35}$ S. A. Schmitz, ${ }^{35}$ L. Sonnenschein, ${ }^{35}$ D. Teyssier,${ }^{35}$ S. Thüer,${ }^{35}$ M. Weber, ${ }^{35}$ V. Cherepanov, ${ }^{36}$ Y. Erdogan, ${ }^{36}$ G. Flügge, ${ }^{36}$ H. Geenen, ${ }^{36}$ M. Geisler, ${ }^{36}$ W. Haj Ahmad, ${ }^{36}$ F. Hoehle, ${ }^{36}$ 
B. Kargoll, ${ }^{36}$ T. Kress,${ }^{36}$ Y. Kuessel,,${ }^{36}$ J. Lingemann, ${ }^{36, c}$ A. Nowack, ${ }^{36}$ I. M. Nugent, ${ }^{36}$ L. Perchalla,${ }^{36}$ O. Pooth,${ }^{36}$ A. Stahl, ${ }^{36}$ I. Asin, ${ }^{37}$ N. Bartosik, ${ }^{37}$ J. Behr, ${ }^{37}$ W. Behrenhoff, ${ }^{37}$ U. Behrens, ${ }^{37}$ A. J. Bell, ${ }^{37}$ M. Bergholz, ${ }^{37, r}$ A. Bethani,${ }^{37}$ K. Borras, ${ }^{37}$ A. Burgmeier, ${ }^{37}$ A. Cakir, ${ }^{37}$ L. Calligaris,${ }^{37}$ A. Campbell, ${ }^{37}$ S. Choudhury, ${ }^{37}$ F. Costanza, ${ }^{37}$ C. Diez Pardos, ${ }^{37}$ S. Dooling,${ }^{37}$ T. Dorland, ${ }^{37}$ G. Eckerlin, ${ }^{37}$ D. Eckstein, ${ }^{37}$ T. Eichhorn, ${ }^{37}$ G. Flucke, ${ }^{37}$ A. Geiser, ${ }^{37}$ A. Grebenyuk, ${ }^{37}$ P. Gunnellini, ${ }^{37}$ S. Habib, ${ }^{37}$ J. Hauk,${ }^{37}$ G. Hellwig, ${ }^{37}$ M. Hempel, ${ }^{37}$ D. Horton, ${ }^{37}$ H. Jung,${ }^{37}$ M. Kasemann, ${ }^{37}$ P. Katsas, ${ }^{37}$ J. Kieseler,${ }^{37}$ C. Kleinwort, ${ }^{37}$ M. Krämer, ${ }^{37}$ D. Krücker, ${ }^{37}$ W. Lange, ${ }^{37}$ J. Leonard,${ }^{37}$ K. Lipka,${ }^{37}$ W. Lohmann, ${ }^{37, r}$ B. Lutz, ${ }^{37}$ R. Mankel, ${ }^{37}$ I. Marfin, ${ }^{37}$ I.-A. Melzer-Pellmann, ${ }^{37}$ A. B. Meyer, ${ }^{37}$ J. Mnich,${ }^{37}$ A. Mussgiller, ${ }^{37}$ S. Naumann-Emme, ${ }^{37}$ O. Novgorodova,${ }^{37}$ F. Nowak, ${ }^{37}$ H. Perrey, ${ }^{37}$ A. Petrukhin,${ }^{37}$ D. Pitzl,${ }^{37}$ R. Placakyte, ${ }^{37}$ A. Raspereza, ${ }^{37}$ P. M. Ribeiro Cipriano, ${ }^{37}$ C. Riedl,${ }^{37}$ E. Ron, ${ }^{37}$ M. O. Sahin, ${ }^{37}$ J. Salfeld-Nebgen, ${ }^{37}$ R. Schmidt, ${ }^{37, \mathrm{r}}$ T. Schoerner-Sadenius, ${ }^{37}$ M. Schröder, ${ }^{37}$ M. Stein, ${ }^{37}$ A. D. R. Vargas Trevino, ${ }^{37}$ R. Walsh,${ }^{37}$ C. Wissing, ${ }^{37}$ M. Aldaya Martin, ${ }^{38}$ V. Blobel,${ }^{38}$ H. Enderle,${ }^{38}$ J. Erfle,${ }^{38}$ E. Garutti, ${ }^{38}$ K. Goebel, ${ }^{38}$ M. Görner, ${ }^{38}$ M. Gosselink, ${ }^{38}$ J. Haller, ${ }^{38}$ R. S. Höing, ${ }^{38}$ H. Kirschenmann, ${ }^{38}$ R. Klanner, ${ }^{38}$ R. Kogler, ${ }^{38}$ J. Lange, ${ }^{38}$ I. Marchesini, ${ }^{38}$ J. Ott, ${ }^{38}$ T. Peiffer, ${ }^{38}$ N. Pietsch, ${ }^{38}$ D. Rathjens, ${ }^{38}$ C. Sander, ${ }^{38}$ H. Schettler,${ }^{38}$ P. Schleper, ${ }^{38}$ E. Schlieckau, ${ }^{38}$ A. Schmidt, ${ }^{38}$ M. Seidel,,${ }^{38}$ J. Sibille,${ }^{38, s}$ V. Sola, ${ }^{38}$ H. Stadie,${ }^{38}$ G. Steinbrück, ${ }^{38}$ D. Troendle,${ }^{38}$ E. Usai, ${ }^{38}$ L. Vanelderen, ${ }^{38}$ C. Barth, ${ }^{39}$ C. Baus, ${ }^{39}$ J. Berger ${ }^{39}$ C. Böser,${ }^{39}$ E. Butz, ${ }^{39}$ T. Chwalek, ${ }^{39}$ W. De Boer, ${ }^{39}$ A. Descroix, ${ }^{39}$ A. Dierlamm, ${ }^{39}$ M. Feindt, ${ }^{39}$ M. Guthoff, ${ }^{39, c}$ F. Hartmann, ${ }^{39, c}$ T. Hauth,${ }^{39, c}$ H. Held, ${ }^{39}$ K. H. Hoffmann, ${ }^{39}$ U. Husemann, ${ }^{39}$ I. Katkov, ${ }^{39, \mathrm{f}}$ A. Kornmayer, ${ }^{39, \mathrm{c}}$ E. Kuznetsova, ${ }^{39}$ P. Lobelle Pardo, ${ }^{39}$ D. Martschei, ${ }^{39}$ M. U. Mozer, ${ }^{39}$ T. Müller, ${ }^{39}$ M. Niegel,${ }^{39}$ A. Nürnberg, ${ }^{39}$ O. Oberst, ${ }^{39}$ G. Quast,${ }^{39}$ K. Rabbertz, ${ }^{39}$ F. Ratnikov,${ }^{39}$ S. Röcker, ${ }^{39}$ F.-P. Schilling, ${ }^{39}$ G. Schott, ${ }^{39}$ H. J. Simonis,${ }^{39}$ F. M. Stober, ${ }^{39}$ R. Ulrich, ${ }^{39}$ J. Wagner-Kuhr, ${ }^{39}$ S. Wayand,${ }^{39}$ T. Weiler, ${ }^{39}$ R. Wolf, ${ }^{39}$ M. Zeise, ${ }^{39}$ G. Anagnostou, ${ }^{40}$ G. Daskalakis, ${ }^{40}$ T. Geralis, ${ }^{40}$ S. Kesisoglou, ${ }^{40}$ A. Kyriakis, ${ }^{40}$ D. Loukas, ${ }^{40}$ A. Markou, ${ }^{40}$ C. Markou, ${ }^{40}$ E. Ntomari, ${ }^{40}$ A. Psallidas, ${ }^{40}$ I. Topsis-giotis, ${ }^{40}$ L. Gouskos,${ }^{41}$ A. Panagiotou ${ }^{41}$ N. Saoulidou, ${ }^{41}$ E. Stiliaris, ${ }^{41}$

X. Aslanoglou, ${ }^{42}$ I. Evangelou, ${ }^{42}$ G. Flouris, ${ }^{42}$ C. Foudas, ${ }^{42}$ P. Kokkas, ${ }^{42}$ N. Manthos, ${ }^{42}$ I. Papadopoulos, ${ }^{42}$ E. Paradas, ${ }^{42}$ G. Bencze, ${ }^{43}$ C. Hajdu, ${ }^{43}$ P. Hidas, ${ }^{43}$ D. Horvath, ${ }^{43, t}$ F. Sikler ${ }^{43}$ V. Veszpremi ${ }^{43}$ G. Vesztergombi, ${ }^{43, u}$ A. J. Zsigmond, ${ }^{43}$ N. Beni,${ }^{44}$ S. Czellar, ${ }^{44}$ J. Molnar, ${ }^{44}$ J. Palinkas, ${ }^{44}$ Z. Szillasi, ${ }^{44}$ J. Karancsi,${ }^{45}$ P. Raics, ${ }^{45}$ Z. L. Trocsanyi, ${ }^{45}$ B. Ujvari ${ }^{45}$ S. K. Swain, ${ }^{46}$ S. B. Beri, ${ }^{47}$ V. Bhatnagar, ${ }^{47}$ N. Dhingra, ${ }^{47}$ R. Gupta, ${ }^{47}$ M. Kaur, ${ }^{47}$ M. Z. Mehta, ${ }^{47}$ M. Mittal,${ }^{47}$ N. Nishu,${ }^{47}$ A. Sharma ${ }^{47}$ J. B. Singh, ${ }^{47}$ A. Kumar, ${ }^{48}$ A. Kumar, ${ }^{48}$ S. Ahuja, ${ }^{48}$ A. Bhardwaj, ${ }^{48}$ B. C. Choudhary, ${ }^{48}$ A. Kumar, ${ }^{48}$ S. Malhotra, ${ }^{48}$ M. Naimuddin, ${ }^{48}$ K. Ranjan, ${ }^{48}$ P. Saxena, ${ }^{48}$ V. Sharma, ${ }^{48}$ R. K. Shivpuri, ${ }^{48}$ S. Banerjee, ${ }^{49}$ S. Bhattacharya, ${ }^{49}$ K. Chatterjee, ${ }^{49}$ S. Dutta, ${ }^{49}$ B. Gomber, ${ }^{49}$ S. Jain, ${ }^{49}$ S. Jain, ${ }^{49}$ R. Khurana, ${ }^{49}$ A. Modak, ${ }^{49}$ S. Mukherjee, ${ }^{49}$ D. Roy, ${ }^{49}$ S. Sarkar ${ }^{49}$ M. Sharan, ${ }^{49}$ A. P. Singh ${ }^{49}$ A. Abdulsalam, ${ }^{50}$ D. Dutta, ${ }^{50}$ S. Kailas, ${ }^{50}$ V. Kumar, ${ }^{50}$ A. K. Mohanty, ${ }^{50, c}$ L. M. Pant ${ }^{50}$ P. Shukla, ${ }^{50}$ A. Topkar, ${ }^{50}$ T. Aziz,${ }^{51}$ R. M. Chatterjee, ${ }^{51}$ S. Ganguly,${ }^{51}$ S. Ghosh,${ }^{51}$ M. Guchait,${ }^{51, v}$ A. Gurtu, ${ }^{51, w}$ G. Kole, ${ }^{51}$ S. Kumar, ${ }^{51}$ M. Maity ${ }^{51, x}$ G. Majumder ${ }^{51}$ K. Mazumdar, ${ }^{51}$ G. B. Mohanty, ${ }^{51}$ B. Parida, ${ }^{51}$ K. Sudhakar ${ }^{51}$ N. Wickramage, ${ }^{51, y}$ S. Banerjee, ${ }^{52}$ S. Dugad ${ }^{52}$ H. Arfaei, ${ }^{53}$ H. Bakhshiansohi,${ }^{53}$ H. Behnamian, ${ }^{53}$ S. M. Etesami, ${ }^{53, z}$ A. Fahim, ${ }^{53, \text { aa }}$ A. Jafari, ${ }^{53}$ M. Khakzad, ${ }^{53}$ M. Mohammadi Najafabadi, ${ }^{53}$ M. Naseri, ${ }^{53}$ S. Paktinat Mehdiabadi, ${ }^{53}$ B. Safarzadeh,${ }^{53, b b}$ M. Zeinali, ${ }^{53}$ M. Grunewald,${ }^{54}$ M. Abbrescia, ${ }^{55,55 b}$ L. Barbone, ${ }^{55 a, 55 b}$ C. Calabria, ${ }^{55 a, 55 b}$ S. S. Chhibra, ${ }^{55 a, 55 b}$ A. Colaleo, ${ }^{55 a}$ D. Creanza,${ }^{55 a, 55 c}$ N. De Filippis, ${ }^{55 a, 55 c}$ M. De Palma,${ }^{55 a, 55 b}$ L. Fiore,${ }^{55 a}$ G. Iaselli, ${ }^{55 a, 55 c}$ G. Maggi, ${ }^{55 a, 55 c}$ M. Maggi, ${ }^{55 a}$ B. Marangelli, ${ }^{55 a, 55 b}$ S. My, ${ }^{55 a, 55 c}$ S. Nuzzo,${ }^{55 a, 55 b}$ N. Pacifico, ${ }^{55 a}$ A. Pompili, ${ }^{55 a, 55 b}$ G. Pugliese, ${ }^{55 a, 55 c}$ R. Radogna, ${ }^{55 a, 55 b}$ G. Selvaggi, ${ }^{55 a, 55 b}$ L. Silvestris, ${ }^{55 a}$ G. Singh,${ }^{55 a, 55 b}$ R. Venditti, ${ }^{55 a, 55 b}$ P. Verwilligen, ${ }^{55 a}$ G. Zito, ${ }^{55 \mathrm{a}}$ G. Abbiendi, ${ }^{56 \mathrm{a}}$ A. C. Benvenuti, ${ }^{56 \mathrm{a}}$ D. Bonacorsi, ${ }^{56 \mathrm{a}, 56 \mathrm{~b}}$ S. Braibant-Giacomelli, ${ }^{56 \mathrm{a}, 56 \mathrm{~b}}$ L. Brigliadori, ${ }^{56 \mathrm{a}, 56 \mathrm{~b}}$

R. Campanini ${ }^{56 a, 56 b}$ P. Capiluppi, ${ }^{56 a, 56 b}$ A. Castro, ${ }^{56,56 b}$ F. R. Cavallo, ${ }^{56 a}$ G. Codispoti, ${ }^{56,56 b}$ M. Cuffiani, ${ }^{56 a, 56 b}$ G. M. Dallavalle, ${ }^{56 \mathrm{a}}$ F. Fabbri, ${ }^{56 \mathrm{a}}$ A. Fanfani, ${ }^{56 \mathrm{a}, 56 \mathrm{~b}}$ D. Fasanella, ${ }^{56,56 \mathrm{~b}}$ P. Giacomelli, ${ }^{56 \mathrm{a}}$ C. Grandi, ${ }^{56 \mathrm{a}}$ L. Guiducci, ${ }^{56 \mathrm{a}, 56 \mathrm{~b}}$ S. Marcellini, ${ }^{56 \mathrm{a}}$ G. Masetti, ${ }^{56 \mathrm{a}}$ M. Meneghelli, ${ }^{56,56 \mathrm{~b}}$ A. Montanari, ${ }^{56 \mathrm{a}}$ F. L. Navarria, ${ }^{56 \mathrm{a}, 56 \mathrm{~b}}$ F. Odorici, ${ }^{56 \mathrm{a}}$ A. Perrotta, ${ }^{56 \mathrm{a}}$ F. Primavera, ${ }^{56 a, 56 b}$ A. M. Rossi,${ }^{56 a, 56 b}$ T. Rovelli, ${ }^{56 a, 56 b}$ G. P. Siroli, ${ }^{56,56 b}$ N. Tosi,${ }^{56 a, 56 b}$ R. Travaglini, ${ }^{56 a, 56 b}$ S. Albergo, ${ }^{57 a, 57 b}$ G. Cappello, ${ }^{57 \mathrm{a}}$ M. Chiorboli, ${ }^{57 \mathrm{a}, 57 \mathrm{~b}}$ S. Costa, ${ }^{57,57 b}$ F. Giordano, ${ }^{57 \mathrm{a}, \mathrm{c}}$ R. Potenza, ${ }^{57 a, 57 b}$ A. Tricomi, ${ }^{57 a, 57 b}$ C. Tuve, ${ }^{57 a, 57 b}$ G. Barbagli, ${ }^{58 \mathrm{a}}$ V. Ciulli, ${ }^{58 \mathrm{a}, 58 \mathrm{~b}}$ C. Civinini, ${ }^{58 \mathrm{a}}$ R. D'Alessandro, ${ }^{58 \mathrm{a}, 58 \mathrm{~b}}$ E. Focardi, ${ }^{58 \mathrm{a}, 58 \mathrm{~b}}$ E. Gallo, ${ }^{58 \mathrm{a}}$ S. Gonzi, ${ }^{58 \mathrm{a}, 58 \mathrm{~b}}$ V. Gori, ${ }^{58 a, 58 b}$ P. Lenzi,${ }^{58 a, 58 b}$ M. Meschini, ${ }^{58 a}$ S. Paoletti, ${ }^{58 a}$ G. Sguazzoni, ${ }^{58 a}$ A. Tropiano, ${ }^{58 a, 58 b}$ L. Benussi, ${ }^{59}$ S. Bianco, ${ }^{59}$ F. Fabbri, ${ }^{59}$ D. Piccolo, ${ }^{59}$ P. Fabbricatore, ${ }^{60 \mathrm{a}}$ R. Ferretti, ${ }^{60 \mathrm{a}, 60 \mathrm{~b}}$ F. Ferro, ${ }^{60 \mathrm{a}}$ M. Lo Vetere,${ }^{60 \mathrm{a}, 60 \mathrm{~b}}$ R. Musenich, ${ }^{60 \mathrm{a}}$ E. Robutti, ${ }^{60 \mathrm{a}}$ S. Tosi, ${ }^{60 a, 60 b}$ A. Benaglia, ${ }^{61 \mathrm{a}}$ M. E. Dinardo, ${ }^{61 \mathrm{a}, 61 \mathrm{~b}}$ S. Fiorendi, ${ }^{61,61 \mathrm{~b}, \mathrm{c}}$ S. Gennai, ${ }^{61 \mathrm{a}}$ A. Ghezzi, ${ }^{61 \mathrm{a}, 61 \mathrm{~b}}$ P. Govoni, ${ }^{61 \mathrm{a}, 61 \mathrm{~b}}$ M. T. Lucchini ${ }^{61 \mathrm{a}, 61 \mathrm{~b}, \mathrm{c}}$ S. Malvezzi, ${ }^{61 \mathrm{a}}$ R. A. Manzoni, ${ }^{61 \mathrm{a}, 61 \mathrm{~b}, \mathrm{c}}$ A. Martelli, ${ }^{61 \mathrm{a}, 61 \mathrm{~b}, \mathrm{c}}$ D. Menasce, ${ }^{61 \mathrm{a}}$ L. Moroni, ${ }^{61 \mathrm{a}}$ M. Paganoni, ${ }^{61 \mathrm{a}, 61 \mathrm{~b}}$ D. Pedrini, ${ }^{61 \mathrm{a}}$ S. Ragazzi, ${ }^{61 \mathrm{a}, 61 \mathrm{~b}}$ N. Redaelli, ${ }^{61 \mathrm{a}}$ T. Tabarelli de Fatis, ${ }^{61 \mathrm{a}, 61 \mathrm{~b}}$ S. Buontempo, ${ }^{62 \mathrm{a}}$ N. Cavallo, ${ }^{62 a, 62 c}$ F. Fabozzi, ${ }^{62 a, 62 c}$ A. O. M. Iorio, ${ }^{62 a, 62 b}$ L. Lista, ${ }^{62 a}$ S. Meola, ${ }^{62 a, 62 d, c}$ M. Merola, ${ }^{62 a}$ P. Paolucci, ${ }^{62 a, c}$ 
P. Azzi, ${ }^{63 a}$ N. Bacchetta, ${ }^{63 a}$ D. Bisello, ${ }^{63 a, 63 b}$ A. Branca, ${ }^{63 a, 63 b}$ R. Carlin, ${ }^{63 a, 63 b}$ P. Checchia ${ }^{63 a}$ T. Dorigo, ${ }^{63 a}$ U. Dosselli, ${ }^{63 a}$ F. Fanzago, ${ }^{63 a}$ M. Galanti, ${ }^{63 a, 63 b, c}$ F. Gasparini, ${ }^{63 a, 63 b}$ U. Gasparini, ${ }^{63 a, 63 b}$ P. Giubilato, ${ }^{63 a, 63 b}$ F. Gonella, ${ }^{63 a}$ A. Gozzelino, ${ }^{63 a}$

K. Kanishchev, ${ }^{63 a, 63 c}$ S. Lacaprara, ${ }^{63 a}$ I. Lazzizzera, ${ }^{63 a, 63 c}$ M. Margoni, ${ }^{63 a, 63 b}$ A. T. Meneguzzo, ${ }^{63 a, 63 b}$ J. Pazzini, ${ }^{63 a, 63 b}$ N. Pozzobon, ${ }^{63 a, 63 b}$ P. Ronchese, ${ }^{63 a, 63 b}$ F. Simonetto,${ }^{63 a, 63 b}$ E. Torassa, ${ }^{63 a}$ M. Tosi, ${ }^{63 a, 63 b}$ P. Zotto, ${ }^{63 a, 63 b}$ A. Zucchetta, ${ }^{63 a, 63 b}$ G. Zumerle, ${ }^{63 a, 63 b}$ M. Gabusi ${ }^{64 a, 64 b}$ S. P. Ratti, ${ }^{64 a, 64 b}$ C. Riccardi, ${ }^{64 a, 64 b}$ P. Vitulo, ${ }^{64 a, 64 b}$ M. Biasini, ${ }^{65 a, 65 b}$ G. M. Bilei, ${ }^{65 a}$ L. Fanò, ${ }^{65 a, 65 b}$ P. Lariccia, ${ }^{65 a, 65 b}$ G. Mantovani,${ }^{65 a, 65 b}$ M. Menichelli, ${ }^{65 a}$ F. Romeo, ${ }^{65 a, 65 b}$ A. Saha, ${ }^{65 a}$ A. Santocchia, ${ }^{65 a, 65 b}$ A. Spiezia, ${ }^{65 a, 65 b}$ K. Androsov, ${ }^{66 a, c c}$ P. Azzurri, ${ }^{66 a}$ G. Bagliesi, ${ }^{66 a}$ J. Bernardini, ${ }^{66 a}$ T. Boccali, ${ }^{66 a}$ G. Broccolo, ${ }^{66 a, 66 c}$

R. Castaldi, ${ }^{66 a}$ M. A. Ciocci, ${ }^{66 a, c c}$ R. Dell'Orso, ${ }^{66 a}$ F. Fiori, ${ }^{66 a, 66 c}$ L. Foà, ${ }^{66 a, 66 c}$ A. Giassi, ${ }^{66 a}$ M. T. Grippo, ${ }^{66 a, c c}$ A. Kraan, ${ }^{66 a}$ F. Ligabue ${ }^{66 a, 66 \mathrm{c}}$ T. Lomtadze, ${ }^{66 \mathrm{a}}$ L. Martini, ${ }^{66 \mathrm{a}, 66 \mathrm{~b}}$ A. Messineo, ${ }^{66 \mathrm{a}, 66 \mathrm{~b}}$ C. S. Moon, ${ }^{66 a, d d}$ F. Palla, ${ }^{66 \mathrm{a}}$ A. Rizzi, ${ }^{66 a, 66 \mathrm{~b}}$ A. Savoy-Navarro, ${ }^{66 a, e e}$ A. T. Serban, ${ }^{66 \mathrm{a}}$ P. Spagnolo, ${ }^{66 \mathrm{a}}$ P. Squillacioti, ${ }^{66 a, c c}$ R. Tenchini, ${ }^{66 \mathrm{a}}$ G. Tonelli, ${ }^{66 a, 66 \mathrm{~b}}$ A. Venturi, ${ }^{66 \mathrm{a}}$ P. G. Verdini, ${ }^{66 \mathrm{a}}$ C. Vernieri, ${ }^{66 a, 66 \mathrm{c}}$ L. Barone, ${ }^{67 \mathrm{a}, 67 \mathrm{~b}}$ F. Cavallari, ${ }^{67 \mathrm{a}}$ D. Del Re,${ }^{67 \mathrm{a}, 67 \mathrm{~b}}$ M. Diemoz,${ }^{67 \mathrm{a}} \mathrm{M}$. Grassi, ${ }^{67 \mathrm{a}, 67 \mathrm{~b}}$ C. Jorda, ${ }^{67 a}$ E. Longo, ${ }^{67,67 b}$ F. Margaroli, ${ }^{67,67 b}$ P. Meridiani, ${ }^{67 a}$ F. Micheli, ${ }^{67 a, 67 b}$ S. Nourbakhsh,${ }^{67 a, 67 b}$ G. Organtini,${ }^{67 a, 67 b}$ R. Paramatti, ${ }^{67 \mathrm{a}}$ S. Rahatlou, ${ }^{67,67 \mathrm{~b}}$ C. Rovelli, ${ }^{67 \mathrm{a}}$ L. Soffi,${ }^{67,67 \mathrm{~b}}$ P. Traczyk,${ }^{67 \mathrm{a}, 67 \mathrm{~b}}$ N. Amapane, ${ }^{68 \mathrm{a}, 68 \mathrm{~b}}$ R. Arcidiacono, ${ }^{68 \mathrm{a}, 68 \mathrm{c}}$ S. Argiro, ${ }^{68 a, 68 b}$ M. Arneodo, ${ }^{68 a, 68 c}$ R. Bellan, ${ }^{68 a, 68 b}$ C. Biino, ${ }^{68 \mathrm{a}}$ N. Cartiglia ${ }^{68 \mathrm{a}}$ S. Casasso, ${ }^{68 \mathrm{a}, 68 \mathrm{~b}}$ M. Costa, ${ }^{68 \mathrm{a}, 68 \mathrm{~b}}$

A. Degano, ${ }^{68 a, 68 b}$ N. Demaria, ${ }^{68 a}$ L. Finco ${ }^{68 a, 68 b}$ M. Machet, ${ }^{68 a, 68 b}$ C. Mariotti, ${ }^{68 a}$ S. Maselli, ${ }^{68 a}$ E. Migliore, ${ }^{68 a, 68 b}$ V. Monaco, ${ }^{68 a, 68 b}$ M. Musich, ${ }^{68 \mathrm{a}}$ M. M. Obertino, ${ }^{68 a, 68 \mathrm{c}}$ G. Ortona ${ }^{68 \mathrm{a}, 68 \mathrm{~b}}$ L. Pacher, ${ }^{68 \mathrm{a}, 68 \mathrm{~b}}$ N. Pastrone ${ }^{68 \mathrm{a}}$ M. Pelliccioni, ${ }^{68 \mathrm{a}, \mathrm{c}}$ G. L. Pinna Angioni, ${ }^{68 a, 68 b}$ A. Romero, ${ }^{68 a}, 68 b$ R. Sacchi ${ }^{68 a, 68 b}$ A. Solano ${ }^{68 a, 68 b}$ A. Staiano, ${ }^{68 a}$ S. Belforte,${ }^{69 a}$ V. Candelise, ${ }^{69 a, 69 b}$ M. Casarsa, ${ }^{69 a}$ F. Cossutti ${ }^{69 a}$ G. Della Ricca, ${ }^{69 a, 69 b}$ B. Gobbo, ${ }^{69 a}$ C. La Licata,${ }^{69 a, 69 b}$ M. Marone ${ }^{69 a, 69 b}$ D. Montanino, ${ }^{69 a, 69 b}$ A. Penzo, ${ }^{69 a}$ A. Schizzi ${ }^{69 a, 69 b}$ T. Umer, ${ }^{69 a, 69 b}$ A. Zanetti, ${ }^{69 a}$ S. Chang, ${ }^{70}$ T. Y. Kim, ${ }^{70}$ S. K. Nam, ${ }^{70}$ D. H. Kim, ${ }^{71}$ G. N. Kim, ${ }^{71}$ J. E. Kim, ${ }^{71}$ D. J. Kong, ${ }^{71}$ S. Lee, ${ }^{71}$ Y. D. Oh, ${ }^{71}$ H. Park, ${ }^{71}$ D. C. Son, ${ }^{71}$ J. Y. Kim, ${ }^{72}$ Z. J. Kim, ${ }^{72}$ S. Song, ${ }^{72}$ S. Choi, ${ }^{73}$ D. Gyun, ${ }^{73}$ B. Hong, ${ }^{73}$ M. Jo, ${ }^{73}$ H. Kim, ${ }^{73}$ Y. Kim, ${ }^{73}$ K. S. Lee, ${ }^{73}$ S. K. Park, ${ }^{73}$ Y. Roh,${ }^{73}$ M. Choi, ${ }^{74}$ J. H. Kim, ${ }^{74}$ C. Park,${ }^{74}$ I. C. Park, ${ }^{74}$ S. Park,${ }^{74}$ G. Ryu,${ }^{74}$ Y. Choi,${ }^{75}$ Y. K. Choi, ${ }^{75}$ J. Goh ${ }^{75}$ M. S. Kim, ${ }^{75}$ E. Kwon, ${ }^{75}$ B. Lee,${ }^{75}$ J. Lee, ${ }^{75}$ S. Lee, ${ }^{75}$ H. Seo, ${ }^{75}$ I. Yu, ${ }^{75}$ A. Juodagalvis, ${ }^{76}$ J. R. Komaragiri, ${ }^{77}$ H. Castilla-Valdez, ${ }^{78}$ E. De La Cruz-Burelo, ${ }^{78}$ I. Heredia-de La Cruz, ${ }^{78, f f}$ R. Lopez-Fernandez, ${ }^{78}$ J. Martínez-Ortega ${ }^{78}$ A. Sanchez-Hernandez, ${ }^{78}$

L. M. Villasenor-Cendejas, ${ }^{78}$ S. Carrillo Moreno, ${ }^{79}$ F. Vazquez Valencia ${ }^{79}$ H. A. Salazar Ibarguen, ${ }^{80}$ E. Casimiro Linares,${ }^{81}$ A. Morelos Pineda, ${ }^{81}$ D. Krofcheck, ${ }^{82}$ P. H. Butler, ${ }^{83}$ R. Doesburg, ${ }^{83}$ S. Reucroft, ${ }^{83}$ H. Silverwood, ${ }^{83}$ M. Ahmad, ${ }^{84}$ M. I. Asghar, ${ }^{84}$ J. Butt, ${ }^{84}$ H. R. Hoorani, ${ }^{84}$ S. Khalid,${ }^{84}$ W. A. Khan, ${ }^{84}$ T. Khurshid,${ }^{84}$ S. Qazi,${ }^{84}$ M. A. Shah,${ }^{84}$ M. Shoaib,${ }^{84}$ H. Bialkowska, ${ }^{85}$ M. Bluj, ${ }^{85, g g}$ B. Boimska, ${ }^{85}$ T. Frueboes, ${ }^{85}$ M. Górski, ${ }^{85}$ M. Kazana ${ }^{85}$ K. Nawrocki, ${ }^{85}$

K. Romanowska-Rybinska, ${ }^{85}$ M. Szleper ${ }^{85}$ G. Wrochna ${ }^{85}$ P. Zalewski, ${ }^{85}$ G. Brona, ${ }^{86}$ K. Bunkowski, ${ }^{86}$ M. Cwiok, ${ }^{86}$ W. Dominik, ${ }^{86}$ K. Doroba ${ }^{86}$ A. Kalinowski, ${ }^{86}$ M. Konecki, ${ }^{86}$ J. Krolikowski, ${ }^{86}$ M. Misiura ${ }^{86}$ W. Wolszczak,${ }^{86}$ P. Bargassa, ${ }^{87}$ C. Beirão Da Cruz E Silva, ${ }^{87}$ P. Faccioli, ${ }^{87}$ P. G. Ferreira Parracho, ${ }^{87}$ M. Gallinaro, ${ }^{87}$ F. Nguyen, ${ }^{87}$ J. Rodrigues Antunes ${ }^{87}$ J. Seixas, ${ }^{87, \mathrm{c}}$ J. Varela ${ }^{87}$ P. Vischia,${ }^{87}$ I. Golutvin, ${ }^{88}$ I. Gorbunov, ${ }^{88}$ A. Kamenev,${ }^{88}$ V. Karjavin, ${ }^{88}$ V. Konoplyanikov,${ }^{88}$ G. Kozlov, ${ }^{88}$ A. Lanev, ${ }^{88}$ A. Malakhov, ${ }^{88}$ V. Matveev ${ }^{88, \text { hh }}$ P. Moisenz ${ }^{88}$ V. Palichik ${ }^{88}$ V. Perelygin, ${ }^{88}$ M. Savina, ${ }^{88}$ S. Shmatov, ${ }^{88}$ S. Shulha, ${ }^{88}$ N. Skatchkov, ${ }^{88}$ V. Smirnov, ${ }^{88}$ A. Zarubin, ${ }^{88}$ V. Golovtsov, ${ }^{89}$ Y. Ivanov, ${ }^{89}$ V. Kim, ${ }^{89}$ P. Levchenko, ${ }^{89}$ V. Murzin, ${ }^{89}$ V. Oreshkin, ${ }^{89}$ I. Smirnov, ${ }^{89}$ V. Sulimov, ${ }^{89}$ L. Uvarov, ${ }^{89}$ S. Vavilov, ${ }^{89}$ A. Vorobyev, ${ }^{89}$ A. Vorobyev,${ }^{89}$ Y. Andreev, ${ }^{90}$ A. Dermenev,${ }^{90}$ S. Gninenko, ${ }^{90}$ N. Golubev, ${ }^{90}$ M. Kirsanov, ${ }^{90}$ N. Krasnikov,${ }^{90}$ A. Pashenkov, ${ }^{90}$ D. Tlisov, ${ }^{90}$ A. Toropin, ${ }^{90}$ V. Epshteyn, ${ }^{91}$ V. Gavrilov,${ }^{91}$ N. Lychkovskaya, ${ }^{91}$ V. Popov, ${ }^{91}$ G. Safronov, ${ }^{91}$ S. Semenov,${ }^{91}$ A. Spiridonov, ${ }^{91}$ V. Stolin, ${ }^{91}$ E. Vlasov,${ }^{91}$ A. Zhokin, ${ }^{91}$ V. Andreev, ${ }^{92}$ M. Azarkin, ${ }^{92}$ I. Dremin, ${ }^{92}$ M. Kirakosyan, ${ }^{92}$ A. Leonidov, ${ }^{92}$ G. Mesyats, ${ }^{92}$ S. V. Rusakov, ${ }^{92}$ A. Vinogradov,${ }^{92}$ A. Belyaev,${ }^{93}$ E. Boos, ${ }^{93}$ V. Bunichev,${ }^{93}$ M. Dubinin, ${ }^{93, h}$ L. Dudko, ${ }^{93}$ A. Ershov, ${ }^{93}$ A. Gribushin,${ }^{93}$ V. Klyukhin, ${ }^{93}$ O. Kodolova, ${ }^{93}$ I. Lokhtin, ${ }^{93}$ S. Obraztsov, ${ }^{93}$ S. Petrushanko, ${ }^{93}$ V. Savrin, ${ }^{93}$ I. Azhgirey, ${ }^{94}$ I. Bayshev,,${ }^{94}$ S. Bitioukov, ${ }^{94}$ V. Kachanov, ${ }^{94}$ A. Kalinin, ${ }^{94}$ D. Konstantinov,${ }^{94}$ V. Krychkine,${ }^{94}$ V. Petrov, ${ }^{94}$ R. Ryutin, ${ }^{94}$ A. Sobol, ${ }^{94}$ L. Tourtchanovitch, ${ }^{94}$ S. Troshin, ${ }^{94}$ N. Tyurin, ${ }^{94}$ A. Uzunian, ${ }^{94}$ A. Volkov, ${ }^{94}$ P. Adzic,${ }^{95, i i}$ M. Djordjevic, ${ }^{95}$ M. Ekmedzic, ${ }^{95}$ J. Milosevic, ${ }^{95}$ M. Aguilar-Benitez, ${ }^{96}$ J. Alcaraz Maestre, ${ }^{96}$ C. Battilana,${ }^{96}$ E. Calvo, ${ }^{96}$ M. Cerrada, ${ }^{96}$ M. Chamizo Llatas, ${ }^{96, c}$ N. Colino, ${ }^{96}$ B. De La Cruz, ${ }^{96}$ A. Delgado Peris, ${ }^{96}$

D. Domínguez Vázquez, ${ }^{96}$ C. Fernandez Bedoya, ${ }^{96}$ J. P. Fernández Ramos, ${ }^{96}$ A. Ferrando, ${ }^{96}$ J. Flix, ${ }^{96}$ M. C. Fouz ${ }^{96}$ P. Garcia-Abia, ${ }^{96}$ O. Gonzalez Lopez,${ }^{96}$ S. Goy Lopez, ${ }^{96}$ J. M. Hernandez, ${ }^{96}$ M. I. Josa, ${ }^{96}$ G. Merino, ${ }^{96}$

E. Navarro De Martino, ${ }^{96}$ J. Puerta Pelayo, ${ }^{96}$ A. Quintario Olmeda, ${ }^{96}$ I. Redondo, ${ }^{96}$ L. Romero, ${ }^{96}$ M. S. Soares,${ }^{96}$

C. Willmott, ${ }^{96}$ C. Albajar, ${ }^{97}$ J. F. de Trocóniz, ${ }^{97}$ H. Brun, ${ }^{98}$ J. Cuevas, ${ }^{98}$ J. Fernandez Menendez, ${ }^{98}$ S. Folgueras, ${ }^{98}$ I. Gonzalez Caballero, ${ }^{98}$ L. Lloret Iglesias, ${ }^{98}$ J. A. Brochero Cifuentes, ${ }^{99}$ I. J. Cabrillo, ${ }^{99}$ A. Calderon, ${ }^{99}$ S. H. Chuang, ${ }^{99}$ 
J. Duarte Campderros, ${ }^{99}$ M. Fernandez, ${ }^{99}$ G. Gomez, ${ }^{99}$ J. Gonzalez Sanchez, ${ }^{99}$ A. Graziano, ${ }^{99}$ A. Lopez Virto, ${ }^{99}$ J. Marco, ${ }^{99}$ R. Marco,${ }^{99}$ C. Martinez Rivero, ${ }^{99}$ F. Matorras, ${ }^{99}$ F. J. Munoz Sanchez, ${ }^{99}$ J. Piedra Gomez,${ }^{99}$ T. Rodrigo, ${ }^{99}$

A. Y. Rodríguez-Marrero, ${ }^{99}$ A. Ruiz-Jimeno, ${ }^{99}$ L. Scodellaro, ${ }^{99}$ I. Vila, ${ }^{99}$ R. Vilar Cortabitarte, ${ }^{99}$ D. Abbaneo, ${ }^{100}$ E. Auffray, ${ }^{100}$ G. Auzinger, ${ }^{100}$ M. Bachtis, ${ }^{100}$ P. Baillon, ${ }^{100}$ A. H. Ball, ${ }^{100}$ D. Barney, ${ }^{100}$ J. Bendavid,${ }^{100}$ L. Benhabib,${ }^{100}$ J. F. Benitez, ${ }^{100}$ C. Bernet, ${ }^{100, i}$ G. Bianchi, ${ }^{100}$ P. Bloch, ${ }^{100}$ A. Bocci, ${ }^{100}$ A. Bonato, ${ }^{100}$ O. Bondu, ${ }^{100}$ C. Botta, ${ }^{100}$ H. Breuker, ${ }^{100}$ T. Camporesi, ${ }^{100}$ G. Cerminara, ${ }^{100}$ T. Christiansen, ${ }^{100}$ J. A. Coarasa Perez, ${ }^{100}$ S. Colafranceschi, ${ }^{100, j j}$ M. D' Alfonso, ${ }^{100}$ D. d'Enterria, ${ }^{100}$ A. Dabrowski, ${ }^{100}$ A. David, ${ }^{100}$ F. De Guio, ${ }^{100}$ A. De Roeck, ${ }^{100}$ S. De Visscher, ${ }^{100}$ S. Di Guida, ${ }^{100}$ M. Dobson, ${ }^{100}$ N. Dupont-Sagorin, ${ }^{100}$ A. Elliott-Peisert, ${ }^{100}$ J. Eugster, ${ }^{100}$ G. Franzoni, ${ }^{100}$ W. Funk, ${ }^{100}$ M. Giffels, ${ }^{100}$ D. Gigi, ${ }^{100}$ K. Gill, ${ }^{100}$ M. Girone,${ }^{100}$ M. Giunta, ${ }^{100}$ F. Glege, ${ }^{100}$ R. Gomez-Reino Garrido, ${ }^{100}$ S. Gowdy ${ }^{100}$ R. Guida, ${ }^{100}$ J. Hammer, ${ }^{100}$ M. Hansen, ${ }^{100}$ P. Harris, ${ }^{100}$ V. Innocente, ${ }^{100}$ P. Janot,${ }^{100}$ E. Karavakis, ${ }^{100}$ K. Kousouris,${ }^{100}$ K. Krajczar, ${ }^{100}$ P. Lecoq, ${ }^{100}$ C. Lourenço, ${ }^{100}$ N. Magini, ${ }^{100}$ L. Malgeri, ${ }^{100}$ M. Mannelli, ${ }^{100}$ L. Masetti, ${ }^{100}$ F. Meijers, ${ }^{100}$ S. Mersi, ${ }^{100}$ E. Meschi ${ }^{100}$ F. Moortgat ${ }^{100}$ M. Mulders ${ }^{100}$ P. Musella, ${ }^{100}$ L. Orsini, ${ }^{100}$ E. Palencia Cortezon, ${ }^{100}$ E. Perez,${ }^{100}$ L. Perrozzi, ${ }^{100}$ A. Petrilli, ${ }^{100}$ G. Petrucciani, ${ }^{100}$ A. Pfeiffer ${ }^{100}$ M. Pierini, ${ }^{100}$ M. Pimiä, ${ }^{100}$ D. Piparo, ${ }^{100}$ M. Plagge, ${ }^{100}$ A. Racz,${ }^{100}$ W. Reece, ${ }^{100}$ G. Rolandi, ${ }^{100, k \mathrm{kk}}$ M. Rovere, ${ }^{100}$ H. Sakulin, ${ }^{100}$ F. Santanastasio, ${ }^{100}$ C. Schäfer, ${ }^{100}$ C. Schwick,${ }^{100}$ S. Sekmen, ${ }^{100}$ A. Sharma, ${ }^{100}$ P. Siegrist, ${ }^{100}$ P. Silva, ${ }^{100}$ M. Simon, ${ }^{100}$ P. Sphicas, ${ }^{100,11}$ J. Steggemann, ${ }^{100}$ B. Stieger ${ }^{100}$ M. Stoye,${ }^{100}$ A. Tsirou, ${ }^{100}$ G. I. Veres, ${ }^{100, u}$ J. R. Vlimant, ${ }^{100}$ H. K. Wöhri, ${ }^{100}$ W. D. Zeuner, ${ }^{100}$ W. Bertl, ${ }^{101}$ K. Deiters, ${ }^{101}$ W. Erdmann, ${ }^{101}$ R. Horisberger, ${ }^{101}$ Q. Ingram, ${ }^{101}$ H. C. Kaestli, ${ }^{101}$ S. König, ${ }^{101}$ D. Kotlinski, ${ }^{101}$ U. Langenegger, ${ }^{101}$ D. Renker, ${ }^{101}$ T. Rohe, ${ }^{101}$ F. Bachmair, ${ }^{102}$ L. Bäni, ${ }^{102}$ L. Bianchini, ${ }^{102}$ P. Bortignon, ${ }^{102}$ M. A. Buchmann, ${ }^{102}$ B. Casal, ${ }^{102}$ N. Chanon, ${ }^{102}$ A. Deisher, ${ }^{102}$ G. Dissertori, ${ }^{102}$ M. Dittmar, ${ }^{102}$ M. Donegà, ${ }^{102}$ M. Dünser, ${ }^{102}$ P. Eller, ${ }^{102}$ C. Grab,${ }^{102}$ D. Hits, ${ }^{102}$ W. Lustermann, ${ }^{102}$ B. Mangano, ${ }^{102}$ A. C. Marini, ${ }^{102}$ P. Martinez Ruiz del Arbol, ${ }^{102}$ D. Meister, ${ }^{102}$ N. Mohr, ${ }^{102}$ C. Nägeli, ${ }^{102, m m}$ P. Nef, ${ }^{102}$ F. Nessi-Tedaldi, ${ }^{102}$ F. Pandolfi, ${ }^{102}$ L. Pape, ${ }^{102}$ F. Pauss, ${ }^{102}$ M. Peruzzi, ${ }^{102}$ M. Quittnat, ${ }^{102}$ F. J. Ronga, ${ }^{102}$ M. Rossini, ${ }^{102}$ A. Starodumov, ${ }^{102, n n}$ M. Takahashi, ${ }^{102}$ L. Tauscher, ${ }^{102, a}$ K. Theofilatos, ${ }^{102}$ D. Treille, ${ }^{102}$ R. Wallny, ${ }^{102}$ H. A. Weber, ${ }^{102}$ C. Amsler, ${ }^{103,00}$ V. Chiochia, ${ }^{103}$ A. De Cosa, ${ }^{103}$ C. Favaro, ${ }^{103}$ A. Hinzmann, ${ }^{103}$ T. Hreus,${ }^{103}$ M. Ivova Rikova, ${ }^{103}$ B. Kilminster ${ }^{103}$ B. Millan Mejias, ${ }^{103}$ J. Ngadiuba, ${ }^{103}$ P. Robmann, ${ }^{103}$ H. Snoek,${ }^{103}$ S. Taroni, ${ }^{103}$ M. Verzetti, ${ }^{103}$ Y. Yang, ${ }^{103}$ M. Cardaci, ${ }^{104}$ K. H. Chen, ${ }^{104}$ C. Ferro, ${ }^{104}$ C. M. Kuo, ${ }^{104}$ S. W. Li, ${ }^{104}$ W. Lin,${ }^{104}$ Y. J. Lu, ${ }^{104}$ R. Volpe,${ }^{104}$ S. S. Yu, ${ }^{104}$ P. Bartalini, ${ }^{105}$ P. Chang, ${ }^{105}$ Y. H. Chang, ${ }^{105}$ Y. W. Chang, ${ }^{105}$ Y. Chao, ${ }^{105}$ K. F. Chen, ${ }^{105}$ P. H. Chen, ${ }^{105}$ C. Dietz, ${ }^{105}$ U. Grundler, ${ }^{105}$ W.-S. Hou, ${ }^{105}$ Y. Hsiung, ${ }^{105}$ K. Y. Kao, ${ }^{105}$ Y. J. Lei, ${ }^{105}$ Y. F. Liu, ${ }^{105}$ R.-S. Lu, ${ }^{105}$ D. Majumder, ${ }^{105}$ E. Petrakou, ${ }^{105}$ X. Shi, ${ }^{105}$ J. G. Shiu, ${ }^{105}$ Y. M. Tzeng, ${ }^{105}$ M. Wang, ${ }^{105}$ R. Wilken, ${ }^{105}$ B. Asavapibhop,${ }^{106}$ N. Suwonjandee, ${ }^{106}$ A. Adiguzel, ${ }^{107}$ M. N. Bakirci, ${ }^{107, p p}$ S. Cerci, ${ }^{107, q 9}$ C. Dozen, ${ }^{107}$ I. Dumanoglu, ${ }^{107}$ E. Eskut, ${ }^{107}$ S. Girgis,${ }^{107}$ G. Gokbulut, ${ }^{107}$ E. Gurpinar, ${ }^{107}$ I. Hos,${ }^{107}$ E. E. Kangal,${ }^{107}$ A. Kayis Topaksu, ${ }^{107}$ G. Onengut, ${ }^{107, \text { rr }}$ K. Ozdemir, ${ }^{107}$ S. Ozturk, ${ }^{107, p p}$ A. Polatoz, ${ }^{107}$ K. Sogut, ${ }^{107, s s}$ D. Sunar Cerci, ${ }^{107, q q}$ B. Tali, ${ }^{107, q q}$ H. Topakli, ${ }^{107, p p}$ M. Vergili, ${ }^{107}$ I. V. Akin, ${ }^{108}$ T. Aliev, ${ }^{108}$ B. Bilin, ${ }^{108}$ S. Bilmis, ${ }^{108}$ M. Deniz, ${ }^{108}$ H. Gamsizkan, ${ }^{108}$ A. M. Guler, ${ }^{108}$ G. Karapinar, ${ }^{108, t t}$ K. Ocalan, ${ }^{108}$ A. Ozpineci, ${ }^{108}$ M. Serin, ${ }^{108}$ R. Sever ${ }^{108}$ U. E. Surat ${ }^{108}$ M. Yalvac, ${ }^{108}$ M. Zeyrek, ${ }^{108}$ E. Gülmez, ${ }^{109}$ B. Isildak, ${ }^{109, \text { uu }}$ M. Kaya, ${ }^{109, v v}$ O. Kaya, ${ }^{109, v v}$ S. Ozkorucuklu, ${ }^{109, w w}$ H. Bahtiyar, ${ }^{110, \mathrm{xx}}$ E. Barlas, ${ }^{110}$ K. Cankocak, ${ }^{110}$ Y. O. Günaydin, ${ }^{110, y y}$ F. I. Vardarlı, ${ }^{110}$ M. Yücel, ${ }^{110}$ L. Levchuk, ${ }^{111}$ P. Sorokin, ${ }^{111}$ J. J. Brooke, ${ }^{112}$ E. Clement, ${ }^{112}$ D. Cussans, ${ }^{112}$ H. Flacher, ${ }^{112}$ R. Frazier, ${ }^{112}$ J. Goldstein, ${ }^{112}$ M. Grimes, ${ }^{112}$ G. P. Heath, ${ }^{112}$ H. F. Heath, ${ }^{112}$ J. Jacob, ${ }^{112}$ L. Kreczko, ${ }^{112}$ C. Lucas, ${ }^{112}$ Z. Meng, ${ }^{112}$ D. M. Newbold, ${ }^{112, z z}$ S. Paramesvaran, ${ }^{112}$ A. Poll, ${ }^{112}$ S. Senkin, ${ }^{112}$ V. J. Smith, ${ }^{112}$ T. Williams, ${ }^{112}$ K. W. Bell, ${ }^{113}$ A. Belyaev, ${ }^{113, \text { aaa }}$ C. Brew, ${ }^{113}$ R. M. Brown, ${ }^{113}$ D. J. A. Cockerill, ${ }^{113}$ J. A. Coughlan, ${ }^{113}$ K. Harder, ${ }^{113}$ S. Harper, ${ }^{113}$ J. Ilic, ${ }^{113}$ E. Olaiya, ${ }^{113}$ D. Petyt, ${ }^{113}$ C. H. Shepherd-Themistocleous, ${ }^{113}$ A. Thea, ${ }^{113}$ I. R. Tomalin, ${ }^{113}$ W. J. Womersley, ${ }^{113}$ S. D. Worm, ${ }^{113}$ M. Baber ${ }^{114}$ R. Bainbridge, ${ }^{114}$ O. Buchmuller, ${ }^{114}$ D. Burton, ${ }^{114}$ D. Colling, ${ }^{114}$ N. Cripps,${ }^{114}$ M. Cutajar, ${ }^{114}$ P. Dauncey, ${ }^{114}$ G. Davies, ${ }^{114}$ M. Della Negra, ${ }^{114}$ W. Ferguson, ${ }^{114}$ J. Fulcher,${ }^{114}$ D. Futyan, ${ }^{114}$ A. Gilbert, ${ }^{114}$

A. Guneratne Bryer, ${ }^{114}$ G. Hall, ${ }^{114}$ Z. Hatherell, ${ }^{114}$ J. Hays,${ }^{114}$ G. Iles, ${ }^{114}$ M. Jarvis, ${ }^{114}$ G. Karapostoli, ${ }^{114}$ M. Kenzie,${ }^{114}$ R. Lane, ${ }^{114}$ R. Lucas, ${ }^{114, z z}$ L. Lyons, ${ }^{114}$ A.-M. Magnan, ${ }^{114}$ J. Marrouche, ${ }^{114}$ B. Mathias, ${ }^{114}$ R. Nandi, ${ }^{114}$ J. Nash, ${ }^{114}$ A. Nikitenko, ${ }^{114, n n}$ J. Pela, ${ }^{114}$ M. Pesaresi,${ }^{114}$ K. Petridis, ${ }^{114}$ M. Pioppi,${ }^{114, b b b}$ D. M. Raymond, ${ }^{114}$ S. Rogerson ${ }^{114}$ A. Rose, ${ }^{114}$ C. Seez, ${ }^{114}$ P. Sharp, ${ }^{114, a}$ A. Sparrow, ${ }^{114}$ A. Tapper, ${ }^{114}$ M. Vazquez Acosta,${ }^{114}$ T. Virdee,${ }^{114}$ S. Wakefield, ${ }^{114}$ N. Wardle, ${ }^{114}$ J. E. Cole, ${ }^{115}$ P. R. Hobson, ${ }^{115}$ A. Khan, ${ }^{115}$ P. Kyberd, ${ }^{115}$ D. Leggat, ${ }^{115}$ D. Leslie, ${ }^{115}$ W. Martin, ${ }^{115}$ I. D. Reid, ${ }^{115}$ P. Symonds, ${ }^{115}$ L. Teodorescu, ${ }^{115}$ M. Turner, ${ }^{115}$ J. Dittmann, ${ }^{116}$ K. Hatakeyama, ${ }^{116}$ A. Kasmi, ${ }^{116}$ H. Liu, ${ }^{116}$

T. Scarborough, ${ }^{116}$ O. Charaf, ${ }^{117}$ S. I. Cooper, ${ }^{117}$ C. Henderson, ${ }^{117}$ P. Rumerio, ${ }^{117}$ A. Avetisyan, ${ }^{118}$ T. Bose,${ }^{118}$ C. Fantasia, ${ }^{118}$ A. Heister, ${ }^{118}$ P. Lawson, ${ }^{118}$ D. Lazic, ${ }^{118}$ J. Rohlf, ${ }^{118}$ D. Sperka, ${ }^{118}$ J. St. John, ${ }^{118}$ L. Sulak, ${ }^{118}$ J. Alimena, ${ }^{119}$ 
S. Bhattacharya, ${ }^{119}$ G. Christopher, ${ }^{119}$ D. Cutts, ${ }^{119}$ Z. Demiragli, ${ }^{119}$ A. Ferapontov, ${ }^{119}$ A. Garabedian, ${ }^{119}$ U. Heintz, ${ }^{119}$ S. Jabeen, ${ }^{119}$ G. Kukartsev, ${ }^{119}$ E. Laird, ${ }^{119}$ G. Landsberg, ${ }^{119}$ M. Luk, ${ }^{119}$ M. Narain, ${ }^{119}$ M. Segala, ${ }^{119}$ T. Sinthuprasith, ${ }^{119}$ T. Speer, ${ }^{119}$ J. Swanson, ${ }^{119}$ R. Breedon, ${ }^{120}$ G. Breto, ${ }^{120}$ M. Calderon De La Barca Sanchez, ${ }^{120}$ S. Chauhan, ${ }^{120}$ M. Chertok, ${ }^{120}$ J. Conway, ${ }^{120}$ R. Conway, ${ }^{120}$ P. T. Cox, ${ }^{120}$ R. Erbacher,${ }^{120}$ M. Gardner, ${ }^{120}$ W. Ko, ${ }^{120}$ A. Kopecky, ${ }^{120}$ R. Lander,${ }^{120}$ T. Miceli, ${ }^{120}$ D. Pellett, ${ }^{120}$ J. Pilot, ${ }^{120}$ F. Ricci-Tam, ${ }^{120}$ B. Rutherford, ${ }^{120}$ M. Searle, ${ }^{120}$ S. Shalhout, ${ }^{120}$ J. Smith, ${ }^{120}$ M. Squires ${ }^{120}$ M. Tripathi, ${ }^{120}$ S. Wilbur, ${ }^{120}$ R. Yohay,${ }^{120}$ V. Andreev, ${ }^{121}$ D. Cline,${ }^{121}$ R. Cousins,${ }^{121}$ S. Erhan, ${ }^{121}$ P. Everaerts, ${ }^{121}$ C. Farrell ${ }^{121}$ M. Felcini,${ }^{121}$ J. Hauser, ${ }^{121}$ M. Ignatenko, ${ }^{121}$ C. Jarvis, ${ }^{121}$ G. Rakness, ${ }^{121}$ P. Schlein, ${ }^{121, a}$ E. Takasugi, ${ }^{121}$ V. Valuev, ${ }^{121}$ M. Weber, ${ }^{121}$ J. Babb, ${ }^{122}$ R. Clare, ${ }^{122}$ J. Ellison, ${ }^{122}$ J. W. Gary, ${ }^{122}$ G. Hanson, ${ }^{122}$ J. Heilman, ${ }^{122}$ P. Jandir, ${ }^{122}$ F. Lacroix, ${ }^{122}$ H. Liu, ${ }^{122}$ O. R. Long, ${ }^{122}$ A. Luthra, ${ }^{122}$ M. Malberti, ${ }^{122}$ H. Nguyen, ${ }^{122}$ A. Shrinivas, ${ }^{122}$ J. Sturdy, ${ }^{122}$ S. Sumowidagdo, ${ }^{122}$ S. Wimpenny, ${ }^{122}$ W. Andrews, ${ }^{123}$ J. G. Branson, ${ }^{123}$ G. B. Cerati, ${ }^{123}$ S. Cittolin, ${ }^{123}$ R. T. D' Agnolo, ${ }^{123}$ D. Evans, ${ }^{123}$ A. Holzner, ${ }^{123}$ R. Kelley, ${ }^{123}$ D. Kovalskyi, ${ }^{123}$ M. Lebourgeois, ${ }^{123}$ J. Letts, ${ }^{123}$ I. Macneill, ${ }^{123}$ S. Padhi, ${ }^{123}$ C. Palmer, ${ }^{123}$ M. Pieri, ${ }^{123}$ M. Sani,${ }^{123}$ V. Sharma, ${ }^{123}$ S. Simon, ${ }^{123}$ E. Sudano, ${ }^{123}$ M. Tadel, ${ }^{123}$ Y. Tu, ${ }^{123}$ A. Vartak, ${ }^{123}$

S. Wasserbaech, ${ }^{123, c c c}$ F. Würthwein, ${ }^{123}$ A. Yagil, ${ }^{123}$ J. Yoo, ${ }^{123}$ D. Barge, ${ }^{124}$ C. Campagnari, ${ }^{124}$ T. Danielson, ${ }^{124}$ K. Flowers, ${ }^{124}$ P. Geffert, ${ }^{124}$ C. George, ${ }^{124}$ F. Golf, ${ }^{124}$ J. Incandela, ${ }^{124}$ C. Justus, ${ }^{124}$ R. Magaña Villalba,${ }^{124}$ N. Mccoll, ${ }^{124}$ V. Pavlunin, ${ }^{124}$ J. Richman, ${ }^{124}$ R. Rossin, ${ }^{124}$ D. Stuart, ${ }^{124}$ W. To,${ }^{124}$ C. West,${ }^{124}$ A. Apresyan, ${ }^{125}$ A. Bornheim, ${ }^{125}$ J. Bunn, ${ }^{125}$ Y. Chen, ${ }^{125}$ E. Di Marco, ${ }^{125}$ J. Duarte,${ }^{125}$ D. Kcira,${ }^{125}$ A. Mott, ${ }^{125}$ H. B. Newman, ${ }^{125}$ C. Pena,${ }^{125}$ C. Rogan, ${ }^{125}$ M. Spiropulu, ${ }^{125}$ V. Timciuc, ${ }^{125}$ R. Wilkinson, ${ }^{125}$ S. Xie, ${ }^{125}$ R. Y. Zhu, ${ }^{125}$ V. Azzolini, ${ }^{126}$ A. Calamba, ${ }^{126}$ R. Carroll, ${ }^{126}$ T. Ferguson, ${ }^{126}$ Y. Iiyama, ${ }^{126}$ D. W. Jang, ${ }^{126}$ M. Paulini, ${ }^{126}$ J. Russ, ${ }^{126}$ H. Vogel, ${ }^{126}$ I. Vorobiev, ${ }^{126}$ J. P. Cumalat, ${ }^{127}$ B. R. Drell, ${ }^{127}$ W. T. Ford, ${ }^{127}$ A. Gaz, ${ }^{127}$ E. Luiggi Lopez, ${ }^{127}$ U. Nauenberg, ${ }^{127}$ J. G. Smith, ${ }^{127}$ K. Stenson, ${ }^{127}$ K. A. Ulmer, ${ }^{127}$ S. R. Wagner, ${ }^{127}$ J. Alexander, ${ }^{128}$ A. Chatterjee, ${ }^{128}$ N. Eggert, ${ }^{128}$ L. K. Gibbons,${ }^{128}$ W. Hopkins, ${ }^{128}$ A. Khukhunaishvili, ${ }^{128}$ B. Kreis, ${ }^{128}$ N. Mirman, ${ }^{128}$ G. Nicolas Kaufman, ${ }^{128}$ J. R. Patterson, ${ }^{128}$ A. Ryd, ${ }^{128}$ E. Salvati, ${ }^{128}$ W. Sun, ${ }^{128}$ W. D. Teo, ${ }^{128}$ J. Thom, ${ }^{128}$ J. Thompson, ${ }^{128}$ J. Tucker, ${ }^{128}$ Y. Weng, ${ }^{128}$ L. Winstrom, ${ }^{128}$ P. Wittich, ${ }^{128}$ D. Winn, ${ }^{129}$ S. Abdullin, ${ }^{130}$ M. Albrow, ${ }^{130}$ J. Anderson, ${ }^{130}$ G. Apollinari, ${ }^{130}$ L. A. T. Bauerdick, ${ }^{130}$ A. Beretvas, ${ }^{130}$ J. Berryhill, ${ }^{130}$ P. C. Bhat, ${ }^{130}$ K. Burkett, ${ }^{130}$ J. N. Butler, ${ }^{130}$ V. Chetluru, ${ }^{130}$ H. W. K. Cheung, ${ }^{130}$ F. Chlebana, ${ }^{130}$ S. Cihangir, ${ }^{130}$ V. D. Elvira, ${ }^{130}$ I. Fisk, ${ }^{130}$ J. Freeman, ${ }^{130}$ Y. Gao, ${ }^{130}$ E. Gottschalk, ${ }^{130}$ L. Gray, ${ }^{130}$ D. Green, ${ }^{130}$ S. Grünendahl, ${ }^{130}$ O. Gutsche, ${ }^{130}$ D. Hare, ${ }^{130}$ R. M. Harris, ${ }^{130}$ J. Hirschauer, ${ }^{130}$ B. Hooberman, ${ }^{130}$ S. Jindariani, ${ }^{130}$ M. Johnson, ${ }^{130}$ U. Joshi, ${ }^{130}$ K. Kaadze, ${ }^{130}$ B. Klima, ${ }^{130}$ S. Kwan, ${ }^{130}$ J. Linacre, ${ }^{130}$ D. Lincoln, ${ }^{130}$ R. Lipton, ${ }^{130}$ J. Lykken, ${ }^{130}$ K. Maeshima, ${ }^{130}$ J. M. Marraffino, ${ }^{130}$ V. I. Martinez Outschoorn, ${ }^{130}$ S. Maruyama, ${ }^{130}$ D. Mason, ${ }^{130}$ P. McBride,${ }^{130} \mathrm{~K}$. Mishra, ${ }^{130}$ S. Mrenna, ${ }^{130}$ Y. Musienko, ${ }^{130, \text { hh }}$ S. Nahn, ${ }^{130}$ C. Newman-Holmes, ${ }^{130}$ V. O’Dell, ${ }^{130}$ O. Prokofyev, ${ }^{130}$ N. Ratnikova, ${ }^{130}$ E. Sexton-Kennedy, ${ }^{130}$ S. Sharma, ${ }^{130}$ W. J. Spalding, ${ }^{130}$ L. Spiegel, ${ }^{130}$ L. Taylor,${ }^{130}$ S. Tkaczyk, ${ }^{130}$ N. V. Tran, ${ }^{130}$ L. Uplegger, ${ }^{130}$ E. W. Vaandering,,${ }^{130}$ R. Vidal, ${ }^{130}$ A. Whitbeck, ${ }^{130}$ J. Whitmore, ${ }^{130}$ W. Wu, ${ }^{130}$ F. Yang, ${ }^{130}$ J. C. Yun, ${ }^{130}$ D. Acosta, ${ }^{131}$ P. Avery, ${ }^{131}$ D. Bourilkov, ${ }^{131}$ T. Cheng, ${ }^{131}$ S. Das, ${ }^{131}$ M. De Gruttola, ${ }^{131}$ G. P. Di Giovanni, ${ }^{131}$ D. Dobur, ${ }^{131}$ R. D. Field, ${ }^{131}$ M. Fisher, ${ }^{131}$ Y. Fu, ${ }^{131}$ I. K. Furic, ${ }^{131}$ J. Hugon, ${ }^{131}$ B. Kim, ${ }^{131}$ J. Konigsberg, ${ }^{131}$ A. Korytov, ${ }^{131}$ A. Kropivnitskaya, ${ }^{131}$ T. Kypreos, ${ }^{131}$ J. F. Low, ${ }^{131}$ K. Matchev, ${ }^{131}$ P. Milenovic, ${ }^{131, \text { ddd }}$ G. Mitselmakher ${ }^{131}$ L. Muniz, ${ }^{131}$ A. Rinkevicius, ${ }^{131}$ L. Shchutska, ${ }^{131}$ N. Skhirtladze,${ }^{131}$ M. Snowball, ${ }^{131}$ J. Yelton, ${ }^{131}$ M. Zakaria, ${ }^{131}$ V. Gaultney, ${ }^{132}$ S. Hewamanage, ${ }^{132}$ S. Linn ${ }^{132}$ P. Markowitz, ${ }^{132}$ G. Martinez, ${ }^{132}$ J. L. Rodriguez, ${ }^{132}$ T. Adams, ${ }^{133}$ A. Askew, ${ }^{133}$ J. Bochenek, ${ }^{133}$ J. Chen, ${ }^{133}$ B. Diamond, ${ }^{133}$ J. Haas, ${ }^{133}$ S. Hagopian, ${ }^{133}$ V. Hagopian, ${ }^{133}$ K. F. Johnson, ${ }^{133}$ H. Prosper, ${ }^{133}$ S. Tentindo, ${ }^{133}$ V. Veeraraghavan, ${ }^{133}$ M. Weinberg, ${ }^{133}$ M. M. Baarmand, ${ }^{134}$ B. Dorney, ${ }^{134}$ M. Hohlmann, ${ }^{134}$ H. Kalakhety, ${ }^{134}$ F. Yumiceva, ${ }^{134}$ M. R. Adams, ${ }^{135}$ L. Apanasevich, ${ }^{135}$ V. E. Bazterra, ${ }^{135}$ R. R. Betts, ${ }^{135}$ I. Bucinskaite, ${ }^{135}$ R. Cavanaugh, ${ }^{135}$ O. Evdokimov, ${ }^{135}$ L. Gauthier, ${ }^{135}$ C. E. Gerber, ${ }^{135}$ D. J. Hofman, ${ }^{135}$ S. Khalatyan, ${ }^{135}$ P. Kurt, ${ }^{135}$ D. H. Moon, ${ }^{135}$ C. O’Brien, ${ }^{135}$ C. Silkworth, ${ }^{135}$ P. Turner, ${ }^{135}$ N. Varelas, ${ }^{135}$ U. Akgun, ${ }^{136}$ E. A. Albayrak ${ }^{136, x x}$ B. Bilki, ${ }^{136, \text { eee }}$ W. Clarida, ${ }^{136}$ K. Dilsiz, ${ }^{136}$ F. Duru, ${ }^{136}$ J.-P. Merlo, ${ }^{136}$ H. Mermerkaya, ${ }^{136, f f f}$ A. Mestvirishvili, ${ }^{136}$ A. Moeller, ${ }^{136}$ J. Nachtman, ${ }^{136}$ H. Ogul, ${ }^{136}$ Y. Onel, ${ }^{136}$ F. Ozok, ${ }^{136, x x}$ S. Sen, ${ }^{136}$ P. Tan, ${ }^{136}$ E. Tiras, ${ }^{136}$ J. Wetzel, ${ }^{136}$ T. Yetkin, ${ }^{136, g g g}$ K. Yi, ${ }^{136}$ I. Anderson, ${ }^{137}$ B. A. Barnett, ${ }^{137}$ B. Blumenfeld, ${ }^{137}$ S. Bolognesi, ${ }^{137}$ D. Fehling, ${ }^{137}$ A. V. Gritsan, ${ }^{137}$ P. Maksimovic, ${ }^{137}$ C. Martin, ${ }^{137}$ M. Swartz,${ }^{137}$ P. Baringer,${ }^{138}$ A. Bean,${ }^{138}$ G. Benelli, ${ }^{138}$ R. P. Kenny III, ${ }^{138}$ M. Murray, ${ }^{138}$ D. Noonan, ${ }^{138}$ S. Sanders, ${ }^{138}$ J. Sekaric, ${ }^{138}$ R. Stringer,${ }^{138}$ Q. Wang, ${ }^{138}$ J. S. Wood, ${ }^{138}$ A. F. Barfuss, ${ }^{139}$ I. Chakaberia, ${ }^{139}$ A. Ivanov, ${ }^{139}$ S. Khalil,,${ }^{139}$ M. Makouski, ${ }^{139}$ Y. Maravin, ${ }^{139}$ L. K. Saini, ${ }^{139}$ S. Shrestha, ${ }^{139}$ I. Svintradze, ${ }^{139}$ J. Gronberg, ${ }^{140}$ D. Lange, ${ }^{140}$ F. Rebassoo, ${ }^{140}$ D. Wright, ${ }^{140}$ A. Baden, ${ }^{141}$ B. Calvert, ${ }^{141}$ S. C. Eno, ${ }^{141}$ J. A. Gomez, ${ }^{141}$ N. J. Hadley ${ }^{141}$ R. G. Kellogg, ${ }^{141}$ T. Kolberg, ${ }^{141}$ Y. Lu, ${ }^{141}$ M. Marionneau, ${ }^{141}$ A. C. Mignerey, ${ }^{141}$ K. Pedro, ${ }^{141}$ A. Skuja, ${ }^{141}$ J. Temple, ${ }^{141}$ M. B. Tonjes, ${ }^{141}$ S. C. Tonwar, ${ }^{141}$ A. Apyan, ${ }^{142}$ R. Barbieri, ${ }^{142}$ G. Bauer, ${ }^{142}$ W. Busza, ${ }^{142}$ I. A. Cali, ${ }^{142}$ 
M. Chan, ${ }^{142}$ L. Di Matteo, ${ }^{142}$ V. Dutta, ${ }^{142}$ G. Gomez Ceballos, ${ }^{142}$ M. Goncharov,${ }^{142}$ D. Gulhan, ${ }^{142}$ M. Klute, ${ }^{142}$ Y. S. Lai, ${ }^{142}$ Y.-J. Lee, ${ }^{142}$ A. Levin, ${ }^{142}$ P. D. Luckey, ${ }^{142}$ T. Ma,${ }^{142}$ C. Paus,${ }^{142}$ D. Ralph,${ }^{142}$ C. Roland,${ }^{142}$ G. Roland, ${ }^{142}$ G. S. F. Stephans, ${ }^{142}$ F. Stöckli, ${ }^{142}$ K. Sumorok, ${ }^{142}$ D. Velicanu, ${ }^{142}$ J. Veverka, ${ }^{142}$ B. Wyslouch, ${ }^{142}$ M. Yang, ${ }^{142}$ A. S. Yoon, ${ }^{142}$ M. Zanetti, ${ }^{142}$ V. Zhukova, ${ }^{142}$ B. Dahmes, ${ }^{143}$ A. De Benedetti, ${ }^{143}$ A. Gude, ${ }^{143}$ S. C. Kao,${ }^{143}$ K. Klapoetke, ${ }^{143}$ Y. Kubota,${ }^{143}$ J. Mans, ${ }^{143}$ N. Pastika, ${ }^{143}$ R. Rusack, ${ }^{143}$ A. Singovsky, ${ }^{143}$ N. Tambe, ${ }^{143}$ J. Turkewitz, ${ }^{143}$ J. G. Acosta, ${ }^{144}$ L. M. Cremaldi, ${ }^{144}$

R. Kroeger ${ }^{144}$ S. Oliveros, ${ }^{144}$ L. Perera ${ }^{144}$ R. Rahmat, ${ }^{144}$ D. A. Sanders, ${ }^{144}$ D. Summers, ${ }^{144}$ E. Avdeeva, ${ }^{145}$ K. Bloom, ${ }^{145}$ S. Bose, ${ }^{145}$ D. R. Claes, ${ }^{145}$ A. Dominguez, ${ }^{145}$ R. Gonzalez Suarez, ${ }^{145}$ J. Keller, ${ }^{145}$ D. Knowlton, ${ }^{145}$ I. Kravchenko, ${ }^{145}$ J. Lazo-Flores, ${ }^{145}$ S. Malik, ${ }^{145}$ F. Meier, ${ }^{145}$ G. R. Snow, ${ }^{145}$ J. Dolen, ${ }^{146}$ A. Godshalk, ${ }^{146}$ I. Iashvili, ${ }^{146}$ S. Jain, ${ }^{146}$ A. Kharchilava, ${ }^{146}$ A. Kumar, ${ }^{146}$ S. Rappoccio, ${ }^{146}$ Z. Wan, ${ }^{146}$ G. Alverson, ${ }^{147}$ E. Barberis, ${ }^{147}$ D. Baumgartel, ${ }^{147}$ M. Chasco,${ }^{147}$ J. Haley, ${ }^{147}$ A. Massironi,${ }^{147}$ D. Nash,${ }^{147}$ T. Orimoto,${ }^{147}$ D. Trocino, ${ }^{147}$ D. Wood, ${ }^{147}$ J. Zhang, ${ }^{147}$ A. Anastassov, ${ }^{148}$ K. A. Hahn, ${ }^{148}$ A. Kubik, ${ }^{148}$ L. Lusito, ${ }^{148}$ N. Mucia, ${ }^{148}$ N. Odell, ${ }^{148}$ B. Pollack, ${ }^{148}$ A. Pozdnyakov, ${ }^{148}$ M. Schmitt, ${ }^{148}$ S. Stoynev, ${ }^{148}$ K. Sung, ${ }^{148}$ M. Velasco, ${ }^{148}$ S. Won, ${ }^{148}$ D. Berry, ${ }^{149}$ A. Brinkerhoff, ${ }^{149}$ K. M. Chan, ${ }^{149}$ A. Drozdetskiy, ${ }^{149}$ M. Hildreth, ${ }^{149}$ C. Jessop, ${ }^{149}$ D. J. Karmgard, ${ }^{149}$ N. Kellams, ${ }^{149}$ J. Kolb, ${ }^{149}$ K. Lannon, ${ }^{149}$ W. Luo, ${ }^{149}$ S. Lynch, ${ }^{149}$ N. Marinelli, ${ }^{149}$ D. M. Morse ${ }^{149}$ T. Pearson, ${ }^{149}$ M. Planer, ${ }^{149}$ R. Ruchti, ${ }^{149}$ J. Slaunwhite, ${ }^{149}$ N. Valls, ${ }^{149}$ M. Wayne,${ }^{149}$ M. Wolf, ${ }^{149}$ A. Woodard, ${ }^{149}$ L. Antonelli, ${ }^{150}$ B. Bylsma, ${ }^{150}$ L. S. Durkin, ${ }^{150}$ S. Flowers, ${ }^{150}$ C. Hill, ${ }^{150}$ R. Hughes, ${ }^{150}$ K. Kotov, ${ }^{150}$ T. Y. Ling, ${ }^{150}$ D. Puigh, ${ }^{150}$ M. Rodenburg, ${ }^{150}$ G. Smith,${ }^{150}$ C. Vuosalo, ${ }^{150}$ B. L. Winer, ${ }^{150}$ H. Wolfe, ${ }^{150}$ H. W. Wulsin, ${ }^{150}$ E. Berry,${ }^{151}$ P. Elmer, ${ }^{151}$ V. Halyo, ${ }^{151}$ P. Hebda,${ }^{151}$ J. Hegeman, ${ }^{151}$ A. Hunt,${ }^{151}$ P. Jindal, ${ }^{151}$ S. A. Koay, ${ }_{151}$ P. Lujan, ${ }^{151}$ D. Marlow, ${ }^{151}$ T. Medvedeva, ${ }^{151}$ M. Mooney, ${ }^{151}$ J. Olsen, ${ }^{151}$ P. Piroué ${ }^{151}$ X. Quan, ${ }^{151}$ A. Raval, ${ }^{151}$ H. Saka, ${ }^{151}$ D. Stickland, ${ }^{151}$ C. Tully, ${ }^{151}$ J. S. Werner, ${ }^{151}$ S. C. Zenz, ${ }^{151}$ A. Zuranski, ${ }^{151}$ E. Brownson, ${ }^{152}$ A. Lopez, ${ }^{152}$ H. Mendez, ${ }^{152}$ J. E. Ramirez Vargas, ${ }^{152}$ E. Alagoz, ${ }^{153}$ D. Benedetti, ${ }^{153}$ G. Bolla, ${ }^{153}$ D. Bortoletto, ${ }^{153}$ M. De Mattia, ${ }^{153}$ A. Everett, ${ }^{153}$ Z. Hu, ${ }^{153}$ M. Jones, ${ }^{153}$ K. Jung, ${ }^{153}$ M. Kress, ${ }^{153}$ N. Leonardo, ${ }^{153}$ D. Lopes Pegna, ${ }^{153}$ V. Maroussov, ${ }^{153}$ P. Merkel, ${ }^{153}$ D. H. Miller, ${ }^{153}$ N. Neumeister, ${ }^{153}$ B. C. Radburn-Smith, ${ }^{153}$ I. Shipsey, ${ }^{153}$ D. Silvers,${ }^{153}$ A. Svyatkovskiy, ${ }^{153}$ F. Wang, ${ }^{153}$ W. Xie, ${ }^{153}$ L. Xu, ${ }^{153}$ H. D. Yoo, ${ }^{153}$ J. Zablocki, ${ }^{153}$ Y. Zheng, ${ }^{153}$ N. Parashar, ${ }^{154}$ A. Adair, ${ }^{155}$ B. Akgun, ${ }^{155}$ K. M. Ecklund, ${ }^{155}$ F. J. M. Geurts, ${ }^{155}$ W. Li, ${ }^{155}$ B. Michlin, ${ }^{155}$ B. P. Padley, ${ }^{155}$ R. Redjimi, ${ }^{155}$ J. Roberts, ${ }^{155}$ J. Zabel, ${ }^{155}$ B. Betchart, ${ }^{156}$ A. Bodek,${ }^{156}$ R. Covarelli, ${ }^{156}$ P. de Barbaro ${ }^{156}$ R. Demina ${ }^{156}$ Y. Eshaq, ${ }^{156}$ T. Ferbel,,${ }^{156}$ A. Garcia-Bellido, ${ }^{156}$ P. Goldenzweig, ${ }^{156}$ J. Han, ${ }^{156}$ A. Harel, ${ }^{156}$ D. C. Miner, ${ }^{156}$ G. Petrillo, ${ }^{156}$ D. Vishnevskiy, ${ }^{156}$ M. Zielinski, ${ }^{156}$ A. Bhatti, ${ }^{157}$ R. Ciesielski, ${ }^{157}$ L. Demortier, ${ }^{157}$ K. Goulianos, ${ }^{157}$ G. Lungu, ${ }^{157}$ S. Malik, ${ }^{157}$ C. Mesropian, ${ }^{157}$ S. Arora, ${ }^{158}$ A. Barker, ${ }^{158}$ J. P. Chou, ${ }^{158}$ C. Contreras-Campana, ${ }^{158}$ E. Contreras-Campana, ${ }^{158}$ D. Duggan, ${ }^{158}$ D. Ferencek, ${ }^{158}$ Y. Gershtein, ${ }^{158}$ R. Gray, ${ }^{158}$ E. Halkiadakis, ${ }^{158}$ D. Hidas, ${ }^{158}$ A. Lath,${ }^{158}$ S. Panwalkar, ${ }^{158}$ M. Park, ${ }^{158}$ R. Patel, ${ }^{158}$ V. Rekovic,${ }^{158}$ J. Robles, ${ }^{158}$ S. Salur, ${ }^{158}$ S. Schnetzer, ${ }^{158}$ C. Seitz, ${ }^{158}$ S. Somalwar, ${ }^{158}$ R. Stone, ${ }^{158}$ S. Thomas, ${ }^{158}$ P. Thomassen, ${ }^{158}$ M. Walker,${ }^{158}$ K. Rose ${ }^{159}$ S. Spanier ${ }^{159}$ Z. C. Yang, ${ }^{159}$ A. York ${ }^{159}$ O. Bouhali, ${ }^{160, \text { hhh }}$ R. Eusebi, ${ }^{160}$ W. Flanagan, ${ }^{160}$ J. Gilmore, ${ }^{160}$ T. Kamon, ${ }^{160, i i i}$ V. Khotilovich, ${ }^{160}$ V. Krutelyov, ${ }^{160}$ R. Montalvo, ${ }^{160}$ I. Osipenkov, ${ }^{160}$ Y. Pakhotin, ${ }^{160}$ A. Perloff, ${ }^{160}$ J. Roe, ${ }^{160}$ A. Safonov, ${ }^{160}$ T. Sakuma, ${ }^{160}$ I. Suarez,${ }^{160}$ A. Tatarinov, ${ }^{160}$ D. Toback, ${ }^{160}$ N. Akchurin, ${ }^{161}$ C. Cowden, ${ }^{161}$ J. Damgov, ${ }^{161}$ C. Dragoiu, ${ }^{161}$ P. R. Dudero, ${ }^{161}$ K. Kovitanggoon, ${ }^{161}$ S. Kunori, ${ }^{161}$ S. W. Lee, ${ }^{161}$ T. Libeiro, ${ }^{161}$ I. Volobouev, ${ }^{161}$ E. Appelt, ${ }^{162}$ A. G. Delannoy, ${ }^{162}$ S. Greene, ${ }^{162}$ A. Gurrola ${ }^{162}$ W. Johns,${ }^{162}$ C. Maguire ${ }^{162}$ Y. Mao, ${ }^{162}$ A. Melo, ${ }^{162}$ M. Sharma ${ }^{162}$ P. Sheldon, ${ }^{162}$ B. Snook, ${ }^{162}$ S. Tuo, ${ }^{162}$ J. Velkovska, ${ }^{162}$ M. W. Arenton, ${ }^{163}$ S. Boutle, ${ }^{163}$ B. Cox, ${ }^{163}$ B. Francis ${ }^{163}$ J. Goodell, ${ }^{163}$ R. Hirosky, ${ }^{163}$ A. Ledovskoy, ${ }^{163}$ C. Lin, ${ }^{163}$ C. Neu, ${ }^{163}$ J. Wood, ${ }^{163}$ S. Gollapinni, ${ }^{164}$ R. Harr, ${ }^{164}$ P. E. Karchin, ${ }^{164}$ C. Kottachchi Kankanamge Don, ${ }^{164}$ P. Lamichhane, ${ }^{164}$ D. A. Belknap, ${ }^{165}$ L. Borrello, ${ }^{165}$ D. Carlsmith, ${ }^{165}$ M. Cepeda, ${ }^{165}$ S. Dasu, ${ }^{165}$ S. Duric, ${ }^{165}$ E. Friis, ${ }^{165}$ M. Grothe, ${ }^{165}$ R. Hall-Wilton, ${ }^{165}$ M. Herndon, ${ }^{165}$ A. Hervé, ${ }^{165}$ P. Klabbers, ${ }^{165}$ J. Klukas, ${ }^{165}$ A. Lanaro, ${ }^{165}$ A. Levine, ${ }^{165}$ R. Loveless, ${ }^{165}$ A. Mohapatra ${ }^{165}$ I. Ojalvo, ${ }^{165}$ T. Perry, ${ }^{165}$ G. A. Pierro, ${ }^{165}$ G. Polese, ${ }^{165}$ I. Ross, ${ }^{165}$ A. Sakharov, ${ }^{165}$ T. Sarangi, ${ }^{165}$ A. Savin ${ }^{165}$ and W. H. Smith ${ }^{165}$

\title{
(CMS Collaboration)
}

\author{
${ }^{1}$ Yerevan Physics Institute, Yerevan, Armenia \\ ${ }^{2}$ Institut für Hochenergiephysik der OeAW, Wien, Austria \\ ${ }^{3}$ National Centre for Particle and High Energy Physics, Minsk, Belarus \\ ${ }^{4}$ Universiteit Antwerpen, Antwerpen, Belgium \\ ${ }^{5}$ Vrije Universiteit Brussel, Brussel, Belgium \\ ${ }^{6}$ Université Libre de Bruxelles, Bruxelles, Belgium
}


${ }^{7}$ Ghent University, Ghent, Belgium

${ }^{8}$ Université Catholique de Louvain, Louvain-la-Neuve, Belgium

${ }^{9}$ Université de Mons, Mons, Belgium

${ }^{10}$ Centro Brasileiro de Pesquisas Fisicas, Rio de Janeiro, Brazil

${ }^{11}$ Universidade do Estado do Rio de Janeiro, Rio de Janeiro, Brazil

${ }^{12 a}$ Universidade Estadual Paulista, São Paulo, Brazil

${ }^{12 \mathrm{~b}}$ Universidade Federal do ABC, São Paulo, Brazil

${ }^{13}$ Institute for Nuclear Research and Nuclear Energy, Sofia, Bulgaria

${ }^{14}$ University of Sofia, Sofia, Bulgaria

${ }^{15}$ Institute of High Energy Physics, Beijing, China

${ }^{16}$ State Key Laboratory of Nuclear Physics and Technology, Peking University, Beijing, China

${ }^{17}$ Universidad de Los Andes, Bogota, Colombia

${ }^{18}$ Technical University of Split, Split, Croatia

${ }^{19}$ University of Split, Split, Croatia

${ }^{20}$ Institute Rudjer Boskovic, Zagreb, Croatia

${ }^{21}$ University of Cyprus, Nicosia, Cyprus

${ }^{22}$ Charles University, Prague, Czech Republic

${ }^{23}$ Academy of Scientific Research and Technology of the Arab Republic of Egypt,

Egyptian Network of High Energy Physics, Cairo, Egypt

${ }^{24}$ National Institute of Chemical Physics and Biophysics, Tallinn, Estonia

${ }^{25}$ Department of Physics, University of Helsinki, Helsinki, Finland

${ }^{26}$ Helsinki Institute of Physics, Helsinki, Finland

${ }^{27}$ Lappeenranta University of Technology, Lappeenranta, Finland

${ }^{28}$ DSM/IRFU, CEA/Saclay, Gif-sur-Yvette, France

${ }^{29}$ Laboratoire Leprince-Ringuet, Ecole Polytechnique, IN2P3-CNRS, Palaiseau, France

${ }^{30}$ Institut Pluridisciplinaire Hubert Curien, Université de Strasbourg,

Université de Haute Alsace Mulhouse, CNRS/IN2P3, Strasbourg, France

${ }^{31}$ Centre de Calcul de l'Institut National de Physique Nucleaire et de Physique des Particules, CNRS/IN2P3, Villeurbanne, France

${ }^{32}$ Université de Lyon, Université Claude Bernard Lyon 1, CNRS-IN2P3,

Institut de Physique Nucléaire de Lyon, Villeurbanne, France

${ }^{33}$ Institute of High Energy Physics and Informatization, Tbilisi State University, Tbilisi, Georgia

${ }^{34}$ RWTH Aachen University, I. Physikalisches Institut, Aachen, Germany

${ }^{35}$ RWTH Aachen University, III. Physikalisches Institut A, Aachen, Germany

${ }^{36}$ RWTH Aachen University, III. Physikalisches Institut B, Aachen, Germany

${ }^{37}$ Deutsches Elektronen-Synchrotron, Hamburg, Germany

${ }^{38}$ University of Hamburg, Hamburg, Germany

${ }^{39}$ Institut für Experimentelle Kernphysik, Karlsruhe, Germany

${ }^{40}$ Institute of Nuclear and Particle Physics (INPP), NCSR Demokritos, Aghia Paraskevi, Greece

${ }^{41}$ University of Athens, Athens, Greece

${ }^{42}$ University of Ioánnina, Ioánnina, Greece

${ }^{43}$ Wigner Research Centre for Physics, Budapest, Hungary

${ }^{44}$ Institute of Nuclear Research ATOMKI, Debrecen, Hungary

${ }^{45}$ University of Debrecen, Debrecen, Hungary

${ }^{46}$ National Institute of Science Education and Research, Bhubaneswar, India

${ }^{47}$ Panjab University, Chandigarh, India

${ }^{48}$ University of Delhi, Delhi, India

${ }^{49}$ Saha Institute of Nuclear Physics, Kolkata, India

${ }^{50}$ Bhabha Atomic Research Centre, Mumbai, India

${ }^{51}$ Tata Institute of Fundamental Research - EHEP, Mumbai, India

${ }^{52}$ Tata Institute of Fundamental Research - HECR, Mumbai, India

${ }^{53}$ Institute for Research in Fundamental Sciences (IPM), Tehran, Iran

${ }^{54}$ University College Dublin, Dublin, Ireland

${ }^{55}$ aNFN Sezione di Bari, Bari, Italy

${ }^{55 \mathrm{~b}}$ Università di Bari, Bari, Italy

${ }^{55 \mathrm{c}}$ Politecnico di Bari, Bari, Italy

${ }^{56 a}$ INFN Sezione di Bologna, Bologna, Italy

${ }^{56 \mathrm{~b}}$ Università di Bologna, Bologna, Italy

${ }^{57 a}$ INFN Sezione di Catania, Catania, Italy

${ }^{57 b}$ Università di Catania, Catania, Italy 


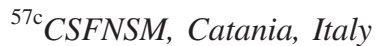

${ }^{58 \mathrm{a}}$ INFN Sezione di Firenze, Firenze, Italy

${ }^{58 \mathrm{~b}}$ Università di Firenze, Firenze, Italy

${ }^{59}$ INFN Laboratori Nazionali di Frascati, Frascati, Italy

${ }^{60 \mathrm{a}}$ INFN Sezione di Genova, Genova, Italy

${ }^{60 \mathrm{~b}}$ Università di Genova, Genova, Italy

${ }^{61 a}$ INFN Sezione di Milano-Bicocca, Milano, Italy

${ }^{61 \mathrm{~b}}$ Università di Milano-Bicocca, Milano, Italy

${ }^{62 \mathrm{a}}$ INFN Sezione di Napoli, Napoli, Italy

${ }^{62 \mathrm{~b}}$ Università di Napoli 'Federico II', Napoli, Italy

${ }^{62 \mathrm{c}}$ Università della Basilicata (Potenza), Napoli, Italy

${ }^{62 \mathrm{~d}}$ Università G. Marconi (Roma), Napoli, Italy

${ }^{63 a}$ INFN Sezione di Padova, Padova, Italy

${ }^{63 \mathrm{~b}}$ Università di Padova, Padova, Italy

${ }^{63 \mathrm{c}}$ Università di Trento (Trento), Padova, Italy

${ }^{64 a}$ INFN Sezione di Pavia, Pavia, Italy

${ }^{64 \mathrm{~b}}$ Università di Pavia, Pavia, Italy

${ }^{65 a}$ INFN Sezione di Perugia

${ }^{65 \mathrm{~b}}$ Università di Perugia

${ }^{66 \mathrm{a}}$ INFN Sezione di Pisa, Pisa, Italy

${ }^{66 \mathrm{~b}}$ Università di Pisa, Pisa, Italy

${ }^{66 \mathrm{c}}$ Scuola Normale Superiore di Pisa, Pisa, Italy

${ }^{67 a}$ INFN Sezione di Roma, Roma, Italy

${ }^{67 \mathrm{~b}}$ Università di Roma, Roma, Italy

${ }^{68 \mathrm{a}}$ INFN Sezione di Torino, Torino, Italy

${ }^{68 \mathrm{~b}}$ Università di Torino, Torino, Italy

${ }^{68 \mathrm{c}}$ Università del Piemonte Orientale (Novara), Torino, Italy

${ }^{69 a}$ INFN Sezione di Trieste, Trieste, Italy

${ }^{69 \mathrm{~b}}$ Università di Trieste, Trieste, Italy

${ }^{70}$ Kangwon National University, Chunchon, Korea

${ }^{71}$ Kyungpook National University, Daegu, Korea

${ }^{72}$ Chonnam National University, Institute for Universe and Elementary Particles, Kwangju, Korea

${ }^{73}$ Korea University, Seoul, Korea

${ }^{74}$ University of Seoul, Seoul, Korea

${ }^{75}$ Sungkyunkwan University, Suwon, Korea

${ }^{76}$ Vilnius University, Vilnius, Lithuania

${ }^{77}$ University of Malaya Jabatan Fizik, Kuala Lumpur, Malaysia

${ }^{78}$ Centro de Investigacion y de Estudios Avanzados del IPN, Mexico City, Mexico

${ }^{79}$ Universidad Iberoamericana, Mexico City, Mexico

${ }^{80}$ Benemerita Universidad Autonoma de Puebla, Puebla, Mexico

${ }^{81}$ Universidad Autónoma de San Luis Potosí, San Luis Potosí, Mexico

${ }^{82}$ University of Auckland, Auckland, New Zealand

${ }^{83}$ University of Canterbury, Christchurch, New Zealand

${ }^{84}$ National Centre for Physics, Quaid-I-Azam University, Islamabad, Pakistan

${ }^{85}$ National Centre for Nuclear Research, Swierk, Poland

${ }^{86}$ Institute of Experimental Physics, Faculty of Physics, University of Warsaw, Warsaw, Poland

${ }^{87}$ Laboratório de Instrumentação e Física Experimental de Partículas, Lisboa, Portugal

${ }^{88}$ Joint Institute for Nuclear Research, Dubna, Russia

${ }^{89}$ Petersburg Nuclear Physics Institute, Gatchina (St. Petersburg), Russia

${ }^{90}$ Institute for Nuclear Research, Moscow, Russia

${ }^{91}$ Institute for Theoretical and Experimental Physics, Moscow, Russia

${ }^{92}$ P.N. Lebedev Physical Institute, Moscow, Russia

${ }^{93}$ Skobeltsyn Institute of Nuclear Physics, Lomonosov Moscow State University, Moscow, Russia

${ }^{94}$ State Research Center of Russian Federation, Institute for High Energy Physics, Protvino, Russia

${ }^{95}$ University of Belgrade, Faculty of Physics and Vinca Institute of Nuclear Sciences, Belgrade, Serbia

${ }^{96}$ Centro de Investigaciones Energéticas Medioambientales y Tecnológicas (CIEMAT), Madrid, Spain

${ }^{97}$ Universidad Autónoma de Madrid, Madrid, Spain

${ }^{98}$ Universidad de Oviedo, Oviedo, Spain

${ }^{99}$ Instituto de Física de Cantabria (IFCA), CSIC-Universidad de Cantabria, Santander, Spain

${ }^{100}$ CERN, European Organization for Nuclear Research, Geneva, Switzerland 
${ }^{101}$ Paul Scherrer Institut, Villigen, Switzerland

${ }^{102}$ Institute for Particle Physics, ETH Zurich, Zurich, Switzerland

${ }^{103}$ Universität Zürich, Zurich, Switzerland

${ }^{104}$ National Central University, Chung-Li, Taiwan

${ }^{105}$ National Taiwan University (NTU), Taipei, Taiwan

${ }^{106}$ Chulalongkorn University, Bangkok, Thailand

${ }^{107}$ Cukurova University, Adana, Turkey

${ }^{108}$ Middle East Technical University, Physics Department, Ankara, Turkey

${ }^{109}$ Bogazici University, Istanbul, Turkey

${ }^{110}$ Istanbul Technical University, Istanbul, Turkey

${ }^{111}$ National Scientific Center, Kharkov Institute of Physics and Technology, Kharkov, Ukraine

${ }^{112}$ University of Bristol, Bristol, United Kingdom

${ }^{113}$ Rutherford Appleton Laboratory, Didcot, United Kingdom

${ }^{114}$ Imperial College, London, United Kingdom

${ }^{115}$ Brunel University, Uxbridge, United Kingdom

${ }^{116}$ Baylor University, Waco, USA

${ }^{117}$ The University of Alabama, Tuscaloosa, USA

${ }^{118}$ Boston University, Boston, USA

${ }^{119}$ Brown University, Providence, USA

${ }^{120}$ University of California, Davis, Davis, USA

${ }^{121}$ University of California, Los Angeles, USA

${ }^{122}$ University of California, Riverside, Riverside, USA

${ }^{123}$ University of California, San Diego, La Jolla, USA

${ }^{124}$ University of California, Santa Barbara, Santa Barbara, USA

${ }^{125}$ California Institute of Technology, Pasadena, USA

${ }^{126}$ Carnegie Mellon University, Pittsburgh, USA

${ }^{127}$ University of Colorado at Boulder, Boulder, USA

${ }^{128}$ Cornell University, Ithaca, USA

${ }^{129}$ Fairfield University, Fairfield, USA

${ }^{130}$ Fermi National Accelerator Laboratory, Batavia, USA

${ }^{131}$ University of Florida, Gainesville, USA

${ }^{132}$ Florida International University, Miami, USA

${ }^{133}$ Florida State University, Tallahassee, USA

${ }^{134}$ Florida Institute of Technology, Melbourne, USA

${ }^{135}$ University of Illinois at Chicago (UIC), Chicago, USA

${ }^{136}$ The University of Iowa, Iowa City, USA

${ }^{137}$ Johns Hopkins University, Baltimore, USA

${ }^{138}$ The University of Kansas, Lawrence, USA

${ }^{139}$ Kansas State University, Manhattan, USA

${ }^{140}$ Lawrence Livermore National Laboratory, Livermore, USA

${ }^{141}$ University of Maryland, College Park, USA

${ }^{142}$ Massachusetts Institute of Technology, Cambridge, USA

${ }^{143}$ University of Minnesota, Minneapolis, USA

${ }^{144}$ University of Mississippi, Oxford, USA

${ }^{145}$ University of Nebraska-Lincoln, Lincoln, USA

${ }^{146}$ State University of New York at Buffalo, Buffalo, USA

${ }^{147}$ Northeastern University, Boston, USA

${ }^{148}$ Northwestern University, Evanston, USA

${ }^{149}$ University of Notre Dame, Notre Dame, USA

${ }^{150}$ The Ohio State University, Columbus, USA

${ }^{151}$ Princeton University, Princeton, USA

${ }^{152}$ University of Puerto Rico, Mayaguez, USA

${ }^{153}$ Purdue University, West Lafayette, USA

${ }^{154}$ Purdue University Calumet, Hammond, USA

${ }^{155}$ Rice University, Houston, USA

${ }^{156}$ University of Rochester, Rochester, USA

${ }^{157}$ The Rockefeller University, New York, USA

${ }^{158}$ Rutgers, The State University of New Jersey, Piscataway, USA

${ }^{159}$ University of Tennessee, Knoxville, USA

${ }^{160}$ Texas A\&M University, College Station, USA 


\footnotetext{
${ }^{161}$ Texas Tech University, Lubbock, USA

${ }^{162}$ Vanderbilt University, Nashville, USA

${ }^{163}$ University of Virginia, Charlottesville, USA

${ }^{164}$ Wayne State University, Detroit, USA

${ }^{165}$ University of Wisconsin, Madison, USA
}

${ }^{\mathrm{a}}$ Deceased.

${ }^{\mathrm{b}}$ Also at Vienna University of Technology, Vienna, Austria.

${ }^{\mathrm{c}}$ Also at CERN, European Organization for Nuclear Research, Geneva, Switzerland.

${ }^{\mathrm{d}}$ Also at Institut Pluridisciplinaire Hubert Curien, Université de Strasbourg, Université de Haute Alsace Mulhouse, CNRS/IN2P3, Strasbourg, France.

${ }^{\mathrm{e}}$ Also at National Institute of Chemical Physics and Biophysics, Tallinn, Estonia.

${ }^{\mathrm{f}}$ Also at Skobeltsyn Institute of Nuclear Physics, Lomonosov Moscow State University, Moscow, Russia.

${ }^{\mathrm{g}}$ Also at Universidade Estadual de Campinas, Campinas, Brazil.

${ }^{\mathrm{h}}$ Also at California Institute of Technology, Pasadena, USA.

${ }^{\mathrm{i}}$ Also at Laboratoire Leprince-Ringuet, Ecole Polytechnique, IN2P3-CNRS, Palaiseau, France.

${ }^{\mathrm{j}}$ Also at Zewail City of Science and Technology, Zewail, Egypt.

${ }^{\mathrm{k}}$ Also at Suez Canal University, Suez, Egypt.

${ }^{1}$ Also at Cairo University, Cairo, Egypt.

${ }^{\mathrm{m}}$ Also at Fayoum University, El-Fayoum, Egypt.

${ }^{\mathrm{n}}$ Also at British University in Egypt, Cairo, Egypt.

${ }^{\circ}$ Present address: Ain Shams University, Cairo, Egypt.

${ }^{\mathrm{p}}$ Also at Université de Haute Alsace, Mulhouse, France.

${ }^{\mathrm{q}}$ Also at Joint Institute for Nuclear Research, Dubna, Russia.

${ }^{\mathrm{r}}$ Also at Brandenburg University of Technology, Cottbus, Germany.

${ }^{\mathrm{s}}$ Also at The University of Kansas, Lawrence, USA.

${ }^{t}$ Also at Institute of Nuclear Research ATOMKI, Debrecen, Hungary.

uAlso at Eötvös Loránd University, Budapest, Hungary.

${ }^{v}$ Also at Tata Institute of Fundamental Research-HECR, Mumbai, India.

${ }^{\mathrm{w}}$ Present address: King Abdulaziz University, Jeddah, Saudi Arabia.

${ }^{\mathrm{x}}$ Also at University of Visva-Bharati, Santiniketan, India.

${ }^{\mathrm{y}}$ Also at University of Ruhuna, Matara, Sri Lanka.

${ }^{\mathrm{z}}$ Also at Isfahan University of Technology, Isfahan, Iran.

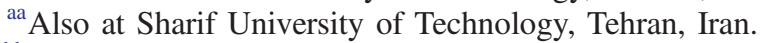

${ }^{\mathrm{bb}}$ Also at Plasma Physics Research Center, Science and Research Branch, Islamic Azad University, Tehran, Iran.

${ }^{c c}$ Also at Università degli Studi di Siena, Siena, Italy.

${ }^{\mathrm{dd}}$ Also at Centre National de la Recherche Scientifique (CNRS)_IN2P3, Paris, France.

${ }^{e e}$ Also at Purdue University, West Lafayette, USA.

${ }^{\mathrm{ff}}$ Also at Universidad Michoacana de San Nicolas de Hidalgo, Morelia, Mexico.

${ }^{\mathrm{gg}}$ Also at National Centre for Nuclear Research, Swierk, Poland.

${ }^{\text {hh }}$ Also at Institute for Nuclear Research, Moscow, Russia.

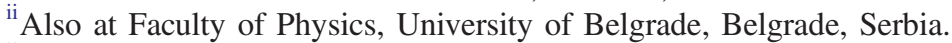

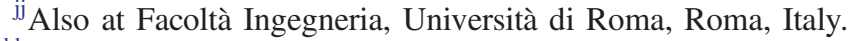

${ }^{\mathrm{kk}}$ Also at Scuola Normale e Sezione dell'INFN, Pisa, Italy.

${ }^{11}$ Also at University of Athens, Athens, Greece.

${ }^{\mathrm{mm}}$ Also at Paul Scherrer Institut, Villigen, Switzerland.

${ }^{n n}$ Also at Institute for Theoretical and Experimental Physics, Moscow, Russia.

${ }^{\text {oo }}$ Also at Albert Einstein Center for Fundamental Physics, Bern, Switzerland.

${ }^{\mathrm{pp}}$ Also at Gaziosmanpasa University, Tokat, Turkey.

${ }^{\mathrm{qq}}$ Also at Adiyaman University, Adiyaman, Turkey.

${ }^{\mathrm{rr}}$ Also at Cag University, Mersin, Turkey.

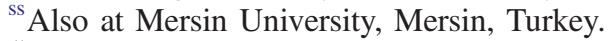

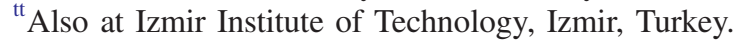

${ }^{\mathrm{uu}}$ Also at Ozyegin University, Istanbul, Turkey.

${ }^{\mathrm{vv}}$ Also at Kafkas University, Kars, Turkey.

${ }^{w w}$ Also at Istanbul University, Faculty of Science, Istanbul, Turkey. 